\title{
WATER-QUALITY RECONNAISSANCE OF GROUND \\ WATER IN THE INHABITED OUTER ISLANDS OF \\ CHUUK STATE, FEDERATED STATES OF MICRONESIA, \\ 1984-85
}

\section{U.S. GEOLOGICAL SURVEY}

Water-Resources Investigations Report 96-4180

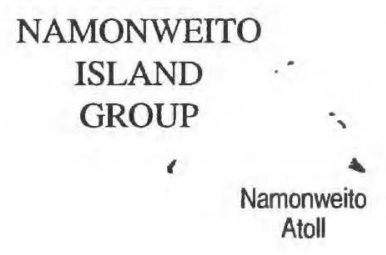

HALL

ISLAND

ISLAND

GROUP
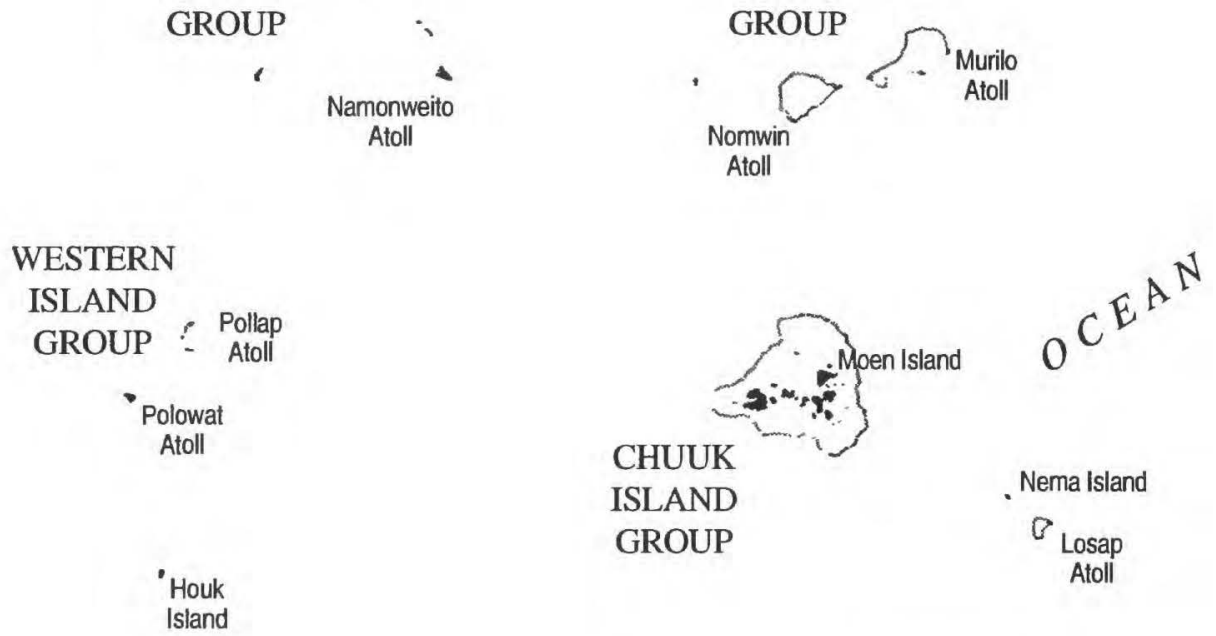

Prepared in cooperation with the

GOVERNMENT OF CHUUK STATE, FEDERATED STATES OF MICRONESIA

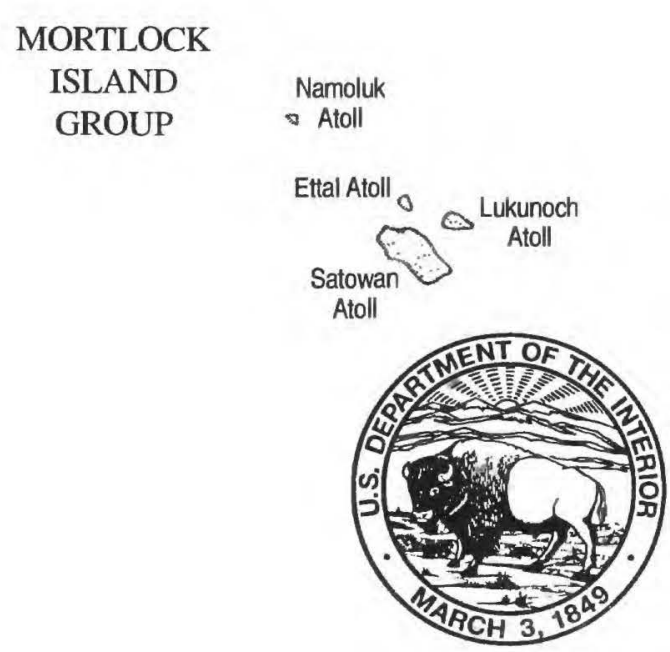




\section{WATER-QUALITY RECONNAISSANCE OF GROUND \\ WATER IN THE INHABITED OUTER ISLANDS OF \\ CHUUK STATE, FEDERATED STATES OF MICRONESIA, 1984-85}

By Scott N. Hamlin and Kiyoshi J. Takasaki

U.S. GEOLOGICAL SURVEY

Water-Resources Investigations Report 96-4180

Prepared in cooperation with the

GOVERNMENT OF CHUUK STATE,

FEDERATED STATES OF MICRONESIA

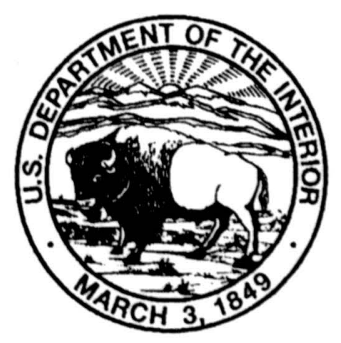

Honolulu, Hawaii

1996 


\title{
U.S. DEPARTMENT OF THE INTERIOR BRUCE BABBITT, Secretary
}

\author{
U.S. GEOLOGICAL SURVEY \\ Gordon P. Eaton, Director
}

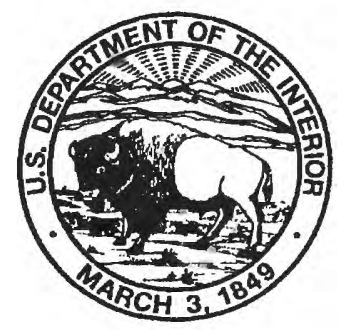

Any use of trade, product, or firm names in this publication is for descriptive purposes only and does not imply endorsement by the U.S. Government

For sale by the U.S. Geological Survey

Branch of Information Services

Box 25286

Denver, CO 80225-0286

For additional information write to:

District Chief

U.S. Geological Survey

677 Ala Moana Blvd., Suite 415

Honolulu, HI 96813 


\section{CONTENTS}

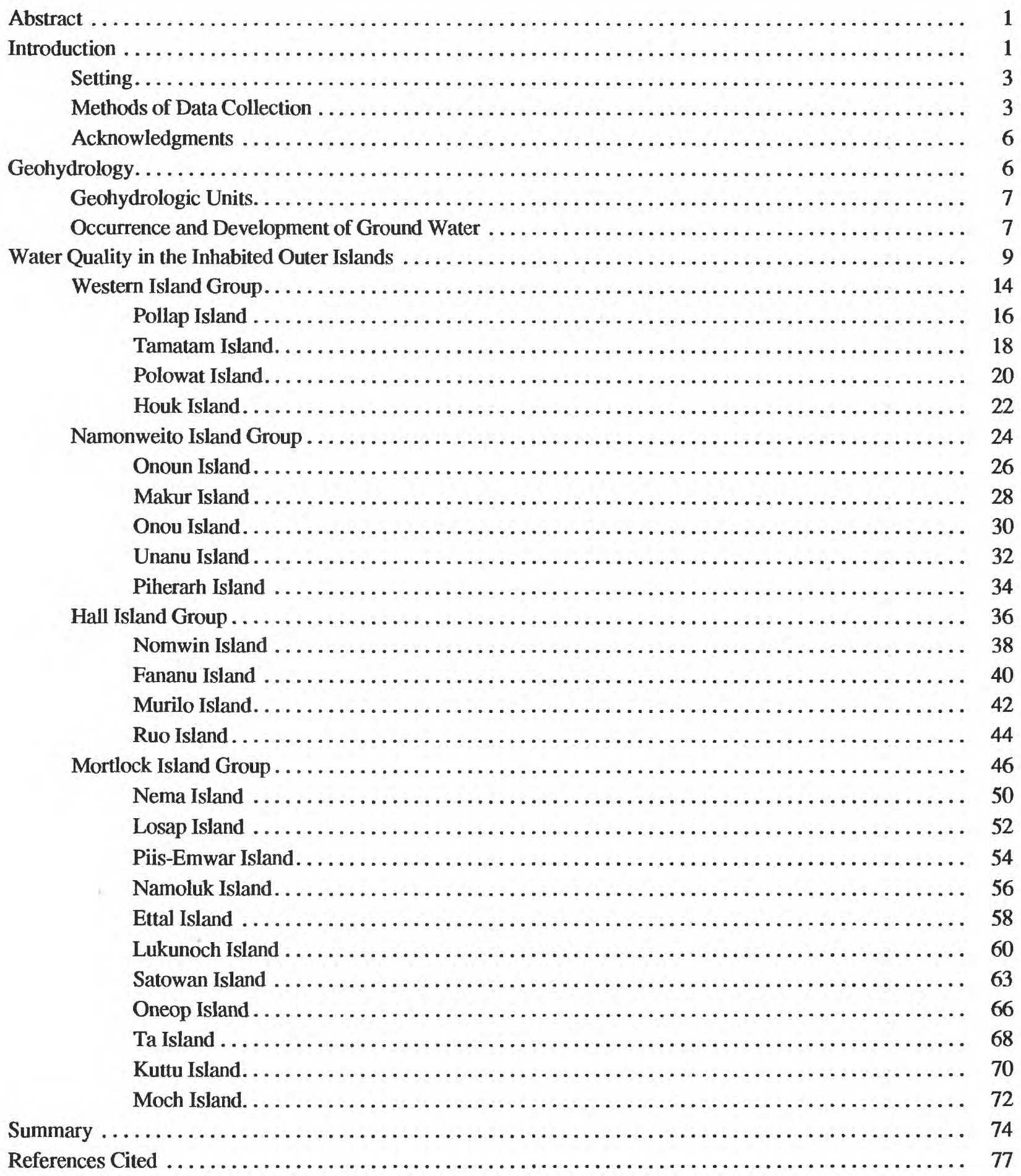




\section{FIGURES}

1-3. Maps showing:

1. Chuuk State, Federated States of Micronesia.......................... 2

2. Island groups in Chuuk State, Federated States of Micronesia $\ldots \ldots \ldots \ldots \ldots \ldots \ldots \ldots \ldots$

3. Distribution of mean annual rainfall in western Pacific.......................... 5

4. Graph showing monthly rainfall for Moen, Polowat, and Lukunoch Islands, Chuuk State, 1987-88 . . . 6

5. Schematic section showing island freshwater lens with transition zone $\ldots \ldots \ldots \ldots \ldots \ldots \ldots \ldots$

6. Schematic drawing of a dug well designed to minimize contamination from the surface $\ldots \ldots \ldots \ldots 10$

7. A trilinear diagram showing the range in composition of ground water from dug wells in inhabited outer islands, Chuuk State, $1984-85 \ldots \ldots \ldots \ldots \ldots \ldots \ldots \ldots \ldots \ldots \ldots \ldots \ldots \ldots \ldots$

8. Graph showing the relation between specific conductance and chloride concentration in ground water from the inhabited outer islands, Chuuk State, $1984-85 \ldots \ldots \ldots \ldots \ldots \ldots \ldots \ldots \ldots \ldots \ldots$

9. Map showing the Western Island Group, Chuuk State, Federated States of Micronesia . . . . . . 15

10-13. Diagrams showing well locations, specific conductance, and nitrate concentrations for:

10. Pollap Island, Pollap Atoll, Western Island Group, Chuuk State, $1984 \ldots \ldots \ldots \ldots \ldots \ldots \ldots 17$

11. Tamatam Island, Pollap Atoll, Western Island Group, Chuuk State, $1984 \ldots \ldots \ldots \ldots \ldots \ldots$

12. Polowat Island, Western Island Group, Chuuk State, $1984 \ldots \ldots \ldots \ldots \ldots \ldots \ldots \ldots \ldots \ldots \ldots \ldots \ldots$

13. Houk Island, Western Island Group, Chuuk State, $1985 \ldots \ldots \ldots \ldots \ldots \ldots \ldots \ldots \ldots \ldots \ldots \ldots \ldots \ldots \ldots \ldots$

14. Map showing the Namonweito Island Group, Chuuk State, Federated States of Micronesia. . . . . . 25

15-19. Diagrams showing well locations, specific conductance, and nitrate concentrations for:

15. Onoun Island, Namonweito Atoll, Namonweito Island Group, Chuuk State, 1984 . . . . . . . 27

16. Makur Island, Namonweito Atoll, Namonweito Island Group, Chuuk State, 1984 . . . . . . . . 29

17. Onou Island, Namonweito Atoll, Namonweito Island Group, Chuuk State, 1984 . . . . . . . . 31

18. Unanu Island, Namonweito Atoll, Namonweito Island Group, Chuuk State, 1984 . . . . . . . 33

19. Piherarh Island, Namonweito Atoll, Namonweito Island Group, Chuuk State, 1984 . . . . . . . 35

20. Map showing the Hall Island Group, Chuuk State, Federated States of Micronesia . . . . . . . . 37

21-24. Diagrams showing well locations, specific conductance, and nitrate concentrations for:

21. Nomwin Island, Nomwin Atoll, Hall Island Group, Chuuk State, $1984 \ldots \ldots \ldots \ldots \ldots \ldots \ldots . .39$

22. Fananu Island, Nomwin Atoll, Hall Island Group, Chuuk State, $1984 \ldots \ldots \ldots \ldots \ldots \ldots \ldots \ldots 41$

23. Murilo Island, Murilo Atoll, Hall Island Group, Chuuk State, $1984 \ldots \ldots \ldots \ldots \ldots \ldots \ldots \ldots . .43$

24. Ruo Island, Murilo Atoll, Hall Island Group, Chuuk State, $1984 \ldots \ldots \ldots \ldots \ldots \ldots \ldots \ldots . \ldots 5$

25. Map showing the Mortlock Island Group, Chuuk State, Federated States of Micronesia ........ 49

26-36. Diagrams showing well locations, specific conductance, and nitrate concentrations for:

26. Nema Island, Mortlock Island Group, Chuuk State, $1985 \ldots \ldots \ldots \ldots \ldots \ldots \ldots \ldots \ldots \ldots$

27. Losap Island, Losap Atoll, Mortlock Island Group, Chuuk State, $1985 \ldots \ldots \ldots \ldots \ldots \ldots \ldots$

28. Piis-Emwar Island, Losap Atoll, Mortlock Island Group, Chuuk State, 1985 . . . . . . . . . 55

29. Namoluk Island, Namoluk Atoll, Mortlock Island Group, Chuuk State, $1984 \ldots \ldots \ldots \ldots \ldots$. 57

30. Ettal Island, Ettal Atoll, Mortlock Island Group, Chuuk State, $1984 \ldots \ldots \ldots \ldots \ldots \ldots \ldots$. . . 59

31. Lukunoch Island, Lukunoch Atoll, Mortlock Island Group, Chuuk State, $1985 \ldots \ldots \ldots \ldots \ldots$

32. Satowan Island, Satowan Atoll, Mortlock Island Group, Chuuk State, $1985 \ldots \ldots \ldots \ldots \ldots \ldots$

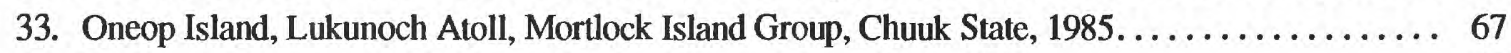

34. Ta Island, Satowan Atoll, Mortlock Island Group, Chuuk State, $1984 \ldots \ldots \ldots \ldots \ldots \ldots \ldots$. . . 69

35. Kuttu Island, Satowan Atoll, Mortlock Island Group, Chuuk State, 1984. . . . . . . . . . 71

36. Moch Island, Satowan Atoll, Mortlock Island Group, Chuuk State, $1984 \ldots \ldots \ldots \ldots \ldots \ldots 73$ 


\section{TABLES}

1. Physical, major ion, and trace element data for selected wells in the Western Island Group,

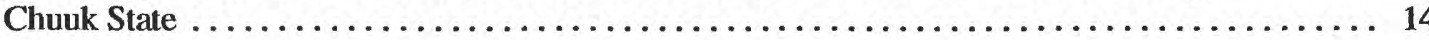

2-5. Selected physical and water-quality data from:

2. Dug wells, February 4 and 6, 1984, Pollap Island, Pollap Atoll, Western Island Group ...... 16

3. Dug wells, February 3, 1984, Tamatam Island, Pollap Atoll, Western Island Group . . . . . . 18

4. Dug and driven wells and taro patch, January 30-31, 1984, Polowat Island, Polowat Atoll, Western Island Group . . . . . . . . . . . . . . . 20

5. Dug wells and miscellaneous sampling sites, February 5, 1984, Houk Island, Polowat Atoll, Western Island Group . . . . . . . . . . . . . . . . . . . 22

6. Physical, major ion, and trace element data for selected wells in the Piherarh and Onoun Islands, Namonweito Atoll Group, Chuuk State . . . . . . . . . . . . . . . . . . . . 24

7-11. Selected physical and water-quality data from:

7. Dug wells and taro patch site, March 23, 1984, Onoun Island, Namonweito Atoll,

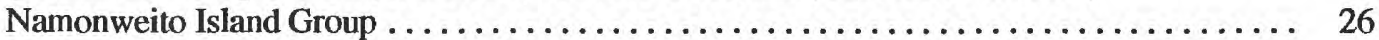

8. Dug wells, March 18, 1984, Makur Island, Namonweito Atoll, Namonweito Island Group . . 28

9. Dug wells and miscellaneous sampling sites, March 19, 1984, Onou Island, Namonweito

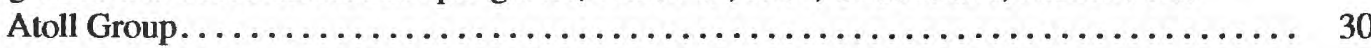

10. Dug and driven wells, March 20, 1984, Unanu Island, Namonweito Atoll, Namonweito Island Group . . . . . . . . . . . . . . 32

11. Dug wells and taro patch site, March 21, 1984, Piherarh Island, Namonweito Atoll, Namonweito Island Group . . . . . . . . . . . . . . . . . . . . . . . . . . .

12. Physical, major ion, and trace element data for selected wells in the Hall Island Group, Chuuk State

13-16. Selected physical and water-quality data from:

13. Dug wells, March 16, 1984, Nomwin Island, Nomwin Atoll, Hall Island Group . . . . . . . 38

14. Dug wells and taro patch sites, March 16, 1984, Fananu Island, Nomwin Atoll,

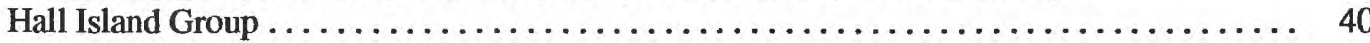

15. Dug wells, March 4, 1984, Murilo Island, Murilo Atoll, Hall Island Group . . . . . . . . . 42

16. Dug wells, March 4, 1984, Ruo Island, Murilo Atoll, Hall Island Group. ............ 44

17. Physical, major ion, and trace element data for selected wells in the Mortlock Island Group,

Chuuk State

18-28. Selected physical and water-quality data from:

18. Dug and driven wells, March 3, 4, and 7, 1984, Nema Island, Mortlock Island Group ....... 50

19. Dug and driven wells, March 6, 1984, Losap Island, Losap Atoll, Mortlock Island Group. . . . . 52

20. Dug and driven wells, March 4-5, 1984, Piis-Emwar Island, Losap Atoll, Mortlock

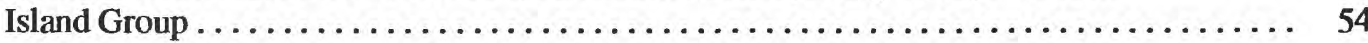

21. Dug and driven wells, February 21, 1984, Namoluk Island, Namoluk Atoll, Mortlock

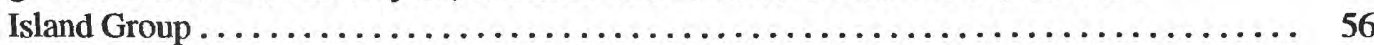

22. Dug wells, February 19, 1984, Ettal Island, Ettal Atoll, Mortlock Island Group .......... 58

23. Dug wells, October 25, 1985, Lukunoch Island, Lukunoch Atoll, Mortlock Island Group . . . . . 60

24. Dug wells, October 22, 23, and 27, 1984, Satowan Island, Satowan Atoll, Mortlock

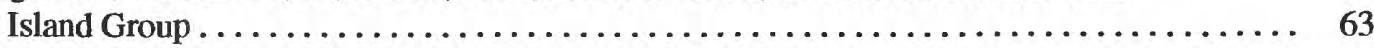

25. Dug wells, February 3, 1984, Oneop Island, Lukunoch Atoll, Mortlock Island Group ....... 66

26. Dug and driven wells, and taro patch site, October 26-27, 1985, Ta Island, Satowan Atoll, Mortlock Island Group . . . . . . . . . . . . . . . . . . . . . . . . . 68

27. Dug wells and taro patch sites, February 17, 1984, Kuttu Island, Satowan Atoll,

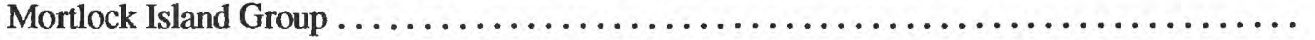


28. Dug wells and taro patch sites, February 18, 1984, Moch Island, Satowan Atoll,

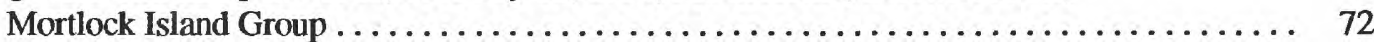

29. Summary of specific conductance data for islands in the Western, Namonweito, Hall, and Mortlock Island Groups, Chuuk State . . . . . . . . . . . . 75

30. Summary of nitrate-concentration data for islands in the Western, Namonweito, Hall, and Mortlock Island Groups, Chuuk State . . . . . . . . . . . . . 76

Conversion Factors

\begin{tabular}{rll}
\hline Multiply & \multicolumn{1}{c}{ By } & To obtain \\
\hline foot (ft) & 0.3048 & meter \\
gallon (gal) & 3.785 & liter \\
gallon per day (gal/d) & 0.00004381 & cubic decimeter per second \\
inch (in.) & 25.4 & millimeter \\
inch (in.) & 2.54 & centimeter \\
inch per year (in/yr) & 2.54 & centimeter per year \\
mile (mi) & 1.609 & kilometer \\
square foot (ft $\left.{ }^{2}\right)$ & 0.09294 & square meter \\
\hline
\end{tabular}

Temperature is given in degrees Fahrenheit $\left({ }^{\circ} \mathrm{F}\right)$, which can be converted to degrees Celsius $\left({ }^{\circ} \mathrm{C}\right)$ by the following equation:

$$
{ }^{\circ} \mathrm{C}=\left({ }^{\circ} \mathrm{F}-32\right) / 1.8
$$

Specific conductance is given in microsiemens per centimeter $(\mu \mathrm{S} / \mathrm{cm})$ at $25^{\circ}$ Celsius. Microsiemens per centimeter is numerically equal to micromhos per centimeter.

Abbreviations used: $\mathrm{mg} / \mathrm{L}$, milligram per liter, $\mu \mathrm{g} / \mathrm{L}$, microgram per liter 


\title{
Water-Quality Reconnaissance of Ground-Water in the Inhabited Outer Islands of Chuuk State, Federated States of Micronesia, 1984-85
}

\author{
By Scott N. Hamlin and Kiyoshi J. Takasaki
}

\section{Abstract}

A reconnaissance of ground-water quality in 24 inhabited outer islands in Chuuk State was made between January 1984 and October 1985. Most of the islands are part of low-lying coral atolls within the Western, Namonuito, Hall, and Mortlock Island Groups. A total of 648 wells were located and sampled for temperature and specific conductance. A few miscellaneous sites such as taro patches also were sampled. The nitrate concentration was determined for 308 water samples. To develop a relation between specific conductance and chloride concentration, the chloride concentration was determined for 63 water samples. In addition, 21 water samples were analyzed for major and trace constituent ion concentrations.

Chloride and nitrate are the primary constituents affecting the potability of ground water in the inhabited outer islands of Chuuk State. The source of chloride in ground water is seawater, whereas nitrate is derived from plant and animal waste materials. The chloride concentrations in many well waters exceed the World Health Organization guideline for drinking water, particularly in wells near the shoreline or on small islands. In addition, the nitrate concentrations in some well waters exceeded the World Health Organization guideline for drinking water.

\section{INTRODUCTION}

The demand for water on the inhabited outer islands of Chuuk State (formerly known as Truk) in the Federated States of Micronesia (fig. 1) is expected to increase as a result of a desire to construct additional sanitary facilities such as showers, flush toilets, and laundry facilities. Water supplies on these islands are obtained from individual and community rainwatercatchment systems and from shallow dug wells that yield fresh to brackish ground water. During extended dry periods the demand for potable water commonly exceeds the supply.

Water-supply shortages on the outer islands of Chuuk State were accentuated during a drought in 1983. Rainfall on the island of Moen in the Chuuk Island Group was only 31 percent of normal for the period October 1982 through May 1983 (van der Brug, 1986). The subnormal rainfall created a severe shortage on the outer islands because most of the potable water on these islands comes from rainwater-catchment systems. In addition to strict rationing of the water supply, it was necessary to use shallow dug wells as a source of drinking water. In some cases, drinking water was shipped to the islands most seriously affected by the drought. One way to alleviate the chronic water-supply shortage would be to further develop ground-water resources for non-potable use so that rainwater can be saved for drinking and cooking.

To help address the water-supply concerns of the inhabited outer islands of Chuuk State, the U.S. Geolog- 

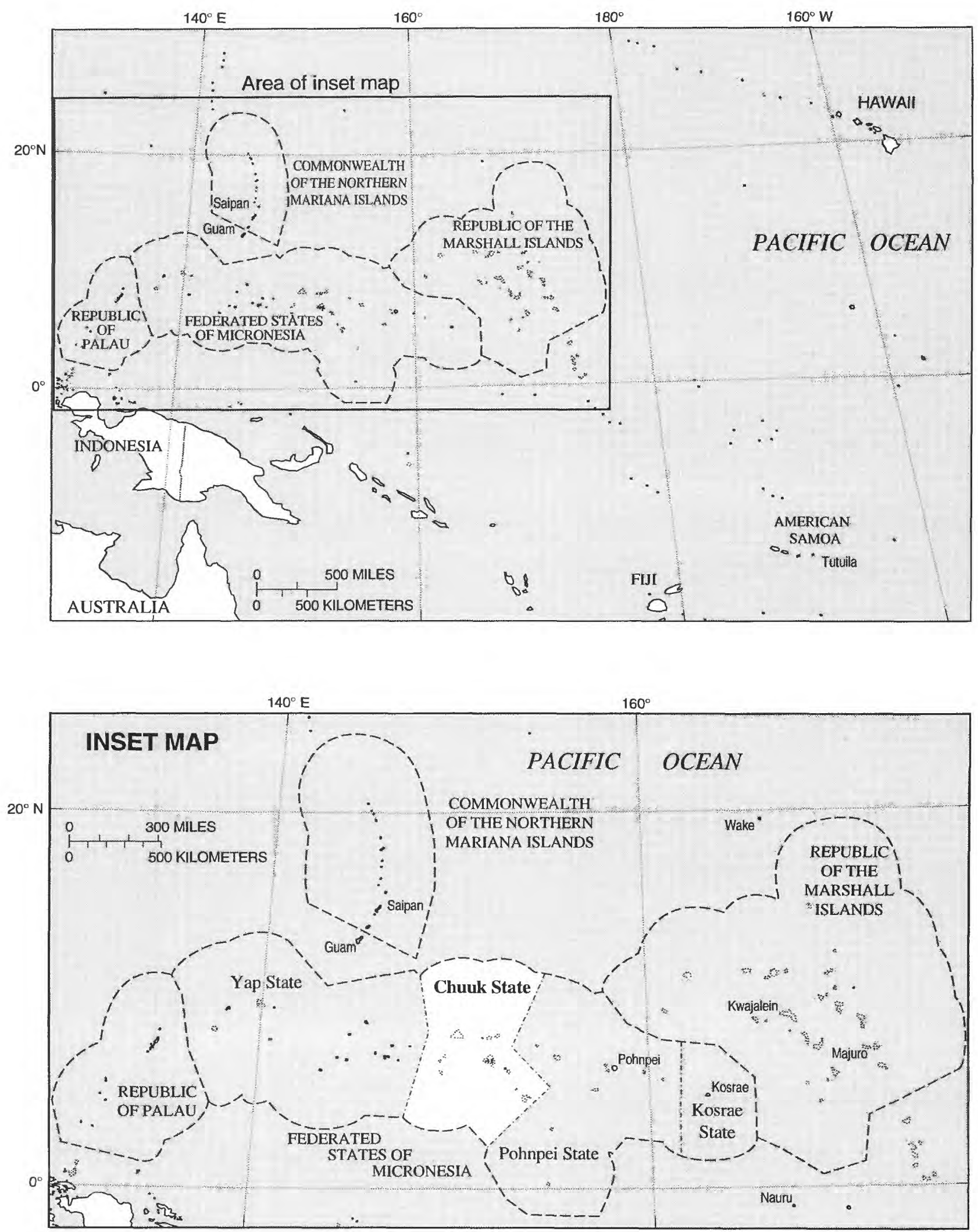

Figure 1. Chuuk State, Federated States of Micronesia. 
ical Survey, in cooperation with Chuuk State, made a ground-water-quality reconnaissance of 24 islands between January 1984 and October 1985. This report documents the results of the reconnaissance survey. The geohydrologic framework of atoll islands is presented as background information.

\section{Setting}

The islands of Chuuk State are located between latitude $4^{\circ}$ to $10^{\circ} \mathrm{N}$ and longitude $148^{\circ}$ to $154^{\circ} \mathrm{E}$ in the western Pacific Ocean, and can be divided into five major island groups (fig. 2). The largest is the Chuuk Island Group, which is a complex of mountainous islands of volcanic origin surrounded by low-lying coral islands. Major outer island groups are the Western, Namonuito, Hall, and Mortlock island groups. The outer island groups of Chuuk State mostly are composed of low-lying coral atoll islands, and are the area of study discussed in this report.

The climate of Chuuk State was described by Stark and others (1958). Mean annual temperature is about $81^{\circ} \mathrm{F}\left(27^{\circ} \mathrm{C}\right)$ and is uniform throughout the year. Relative humidity ranges from about 55 to 100 percent and generally is more than 75 percent. Rainfall is heavy, averaging about $140 \mathrm{in} / \mathrm{yr}$; however, droughts are common. Chuuk State lies in an elongated area of high rainfall centered about $100 \mathrm{mi}$ south of Pohnpei at about latitude $5^{\circ} \mathrm{N}$ and longitude $159^{\circ} \mathrm{E}$ (fig. 3). The area of high rainfall, with more than $140 \mathrm{in} / \mathrm{yr}$, extends eastward beyond Majuro and westward to Palau for a distance of more than $2,000 \mathrm{mi}$. North and south the area of high rainfall extends for a total of about $300 \mathrm{mi}$. The mean annual rainfall for the Chuuk Island Group ranges from about 100 to $180 \mathrm{in}$. Data from the National Weather Service rain gage at Moen Airport, for the 33year period 1952-85, indicate that the area receives an average of about $140 \mathrm{in} / \mathrm{yr}$ of rainfall. Most of the year is wet, except for the period from January through March when the monthly rainfall is only about half that of the other 9 months.

The amount and distribution of rainfall in the outer islands of Chuuk State probably are not significantly different from that of Moen. The regional rainfall map (fig. 3) shows that the rainfall in the outer islands is higher to the south and east and lower to the north and west of the Chuuk Island Group. However, considerable short-term variability in rainfall can be seen in a com- parison of monthly rainfall data for Moen in the Chuuk Island Group, Polowat Atoll in the Western Island Group, and Lukunoch Atoll in the Mortlock Island Group for the period January 1987 through December 1988 (fig. 4). Polowat and Lukunoch Atolls are about 185 miles west and south of Moen, respectively.

Although typhoons are not common, several have passed near or over the islands of Chuuk State.

Typhoons have caused loss of life, widespread damage to buildings and crops, and wave damage to shoreline and coastal structures.

\section{Methods of Data Collection}

The reconnaissance survey of the inhabited outer islands of Chuuk State began in January 1984 and was completed in October 1985. The reconnaissance was limited to the stay of the interisland (field) ship that visits each island once every 4 to 6 weeks. This and another similar ship provide the principal means of transporting people and cargo between the administrative center on Moen in the Chuuk Island Group and the outer islands in the Western, Namonuito, Hall, and Mortlock Island Groups. The duration of stay at each island was normally 1 day. Occasionally, the visits ranged from as short as one-half day to as long as several days.

An inventory of all known dug wells on each of the inhabited islands was made. The water from each well was sampled for measurement of temperature and specific conductance. Selected wells were sampled for measurement of $\mathrm{pH}$, and for analysis of chloride and nitrate concentrations. At least one well in each island group was sampled for analysis of major and trace constituent ion concentrations. A spot sampling was made of ground water in taro patches, especially where the water was reported to be brackish. On at least one island in each island group, a well point was driven and a hand pump installed for demonstration purposes.

Dip samples were collected from dug wells and taro patches. The driven wells installed during the survey were sampled with a hand pump after the specific conductance of the pumped water stabilized and clarity was attained indicating sufficient well development. Temperature, $\mathrm{pH}$, specific conductance, and nitrite concentration were determined in the field. Samples for major and trace constituent ion analysis were preserved for analysis at the U.S. Geological Survey's Central Laboratory (Denver, Colorado). 


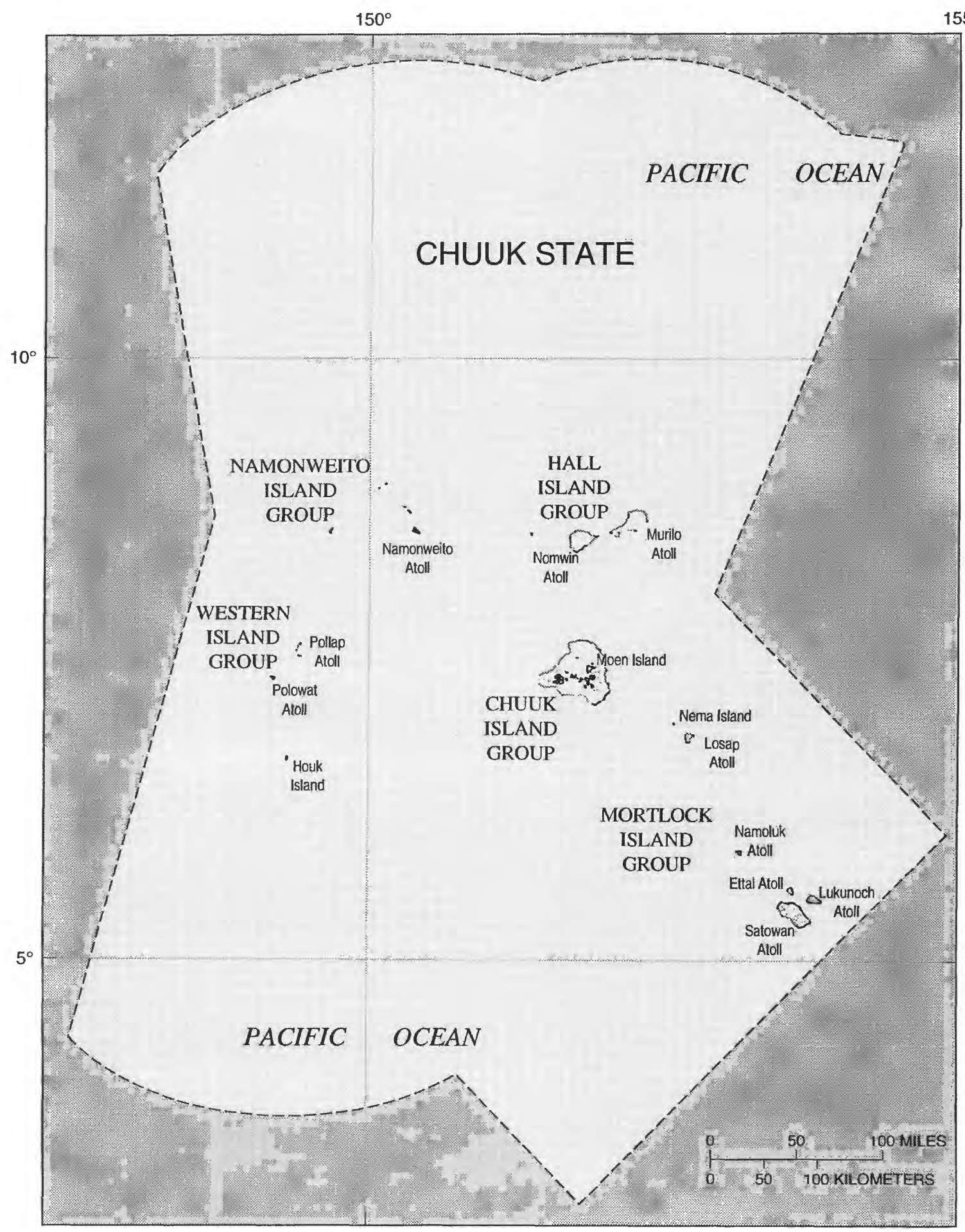

Figure 2. Island groups in Chuuk State, Federated States of Micronesia. 


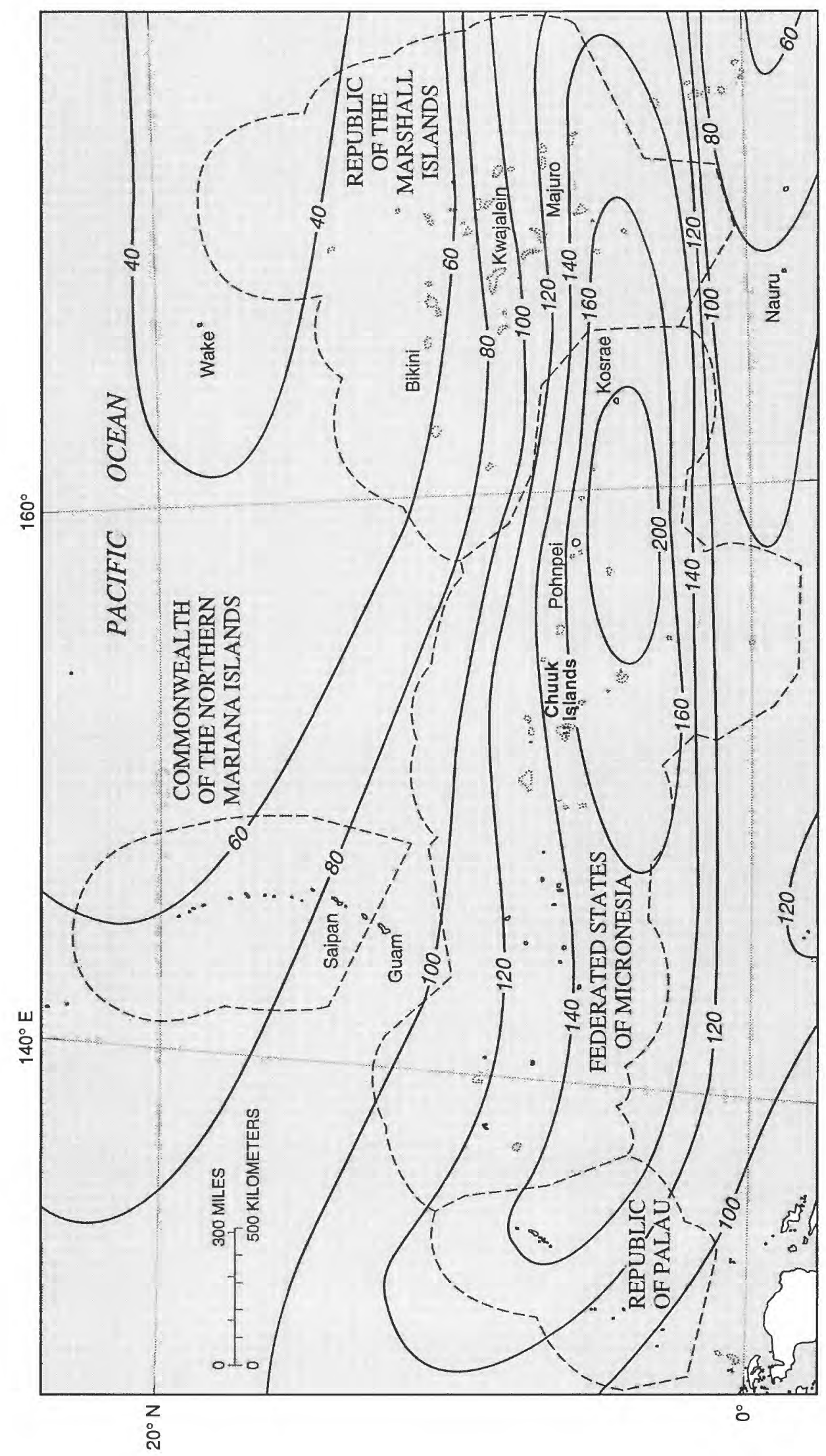

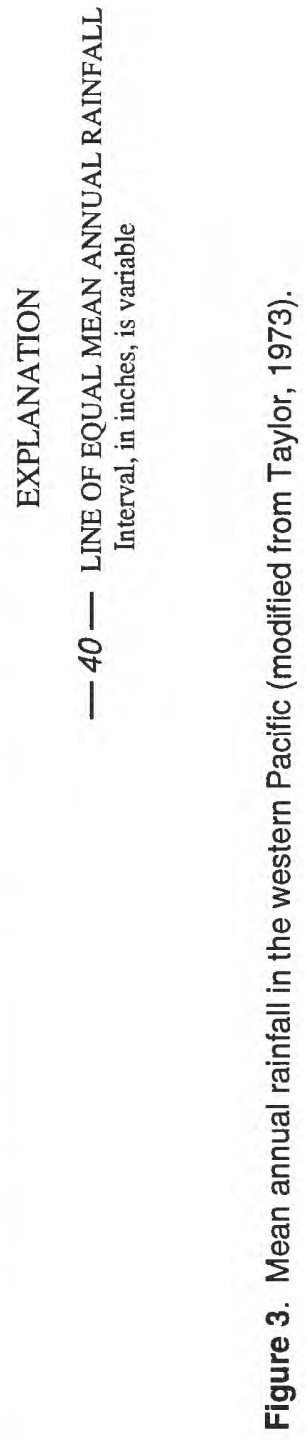




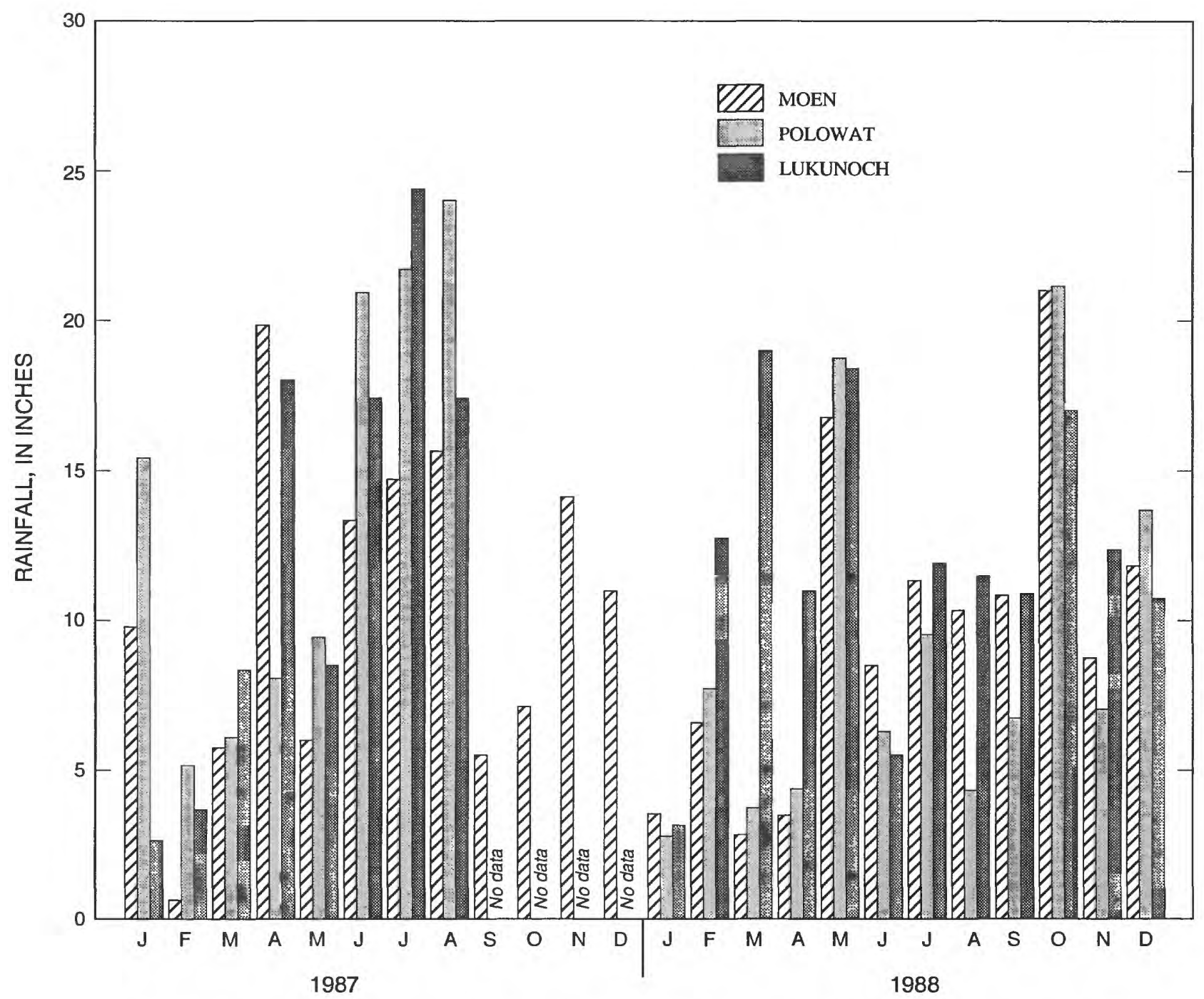

Figure 4. Monthly rainfall for Moen, Polowat, and Lukunoch Islands, Chuuk State, 1987-88 (data from National Oceanic and Atmospheric Administration, 1987, 1988).

\section{Acknowledgments}

The Department of Public Works on the island of Moen in the Chuuk Island Group provided assistance in: (1) scheduling transportation to and from the outer islands, (2) installing well points, and (3) collecting hydrologic data. The Department of Transportation provided itineraries for trips to and from the outer islands and helped coordinate field surveys. Special appreciation is given to the Chief Magistrates of the outer islands, and to the individual well owners, for their assistance in locating wells and facilitating the collection of hydrologic data.

\section{GEOHYDROLOGY}

Most of the outer islands in the Chuuk State are part of low-lying coral atolls. Atolls are sub-circular reefs composed of a resistant framework of calcareous skeletons enclosing a lagoon from the open sea. The upper surface of the reef structure is flat and is constantly scoured and planed off by wave action and dissected 
by seaward-trending surge channels. The reef structure is derived from fringing reefs that surround a volcanic island. As the volcanic island sinks under its own weight, reef organisms build upward in an attempt to keep pace with the relative rise in sea level. The volcanic island eventually is submerged, leaving only the reef and related unconsolidated sediments that form atoll islands.

As part of the environmental studies made in the Marshall Islands in connection with atomic-bomb testing, the U.S. Navy drilled a series of deep test holes on Enewetak Atoll. Two of the test holes went through a 3,900-ft cap of shallow-water reef limestone and bottomed in volcanic material (basalt). The structure and composition of the shallow-water reef limestones forming the atoll were found to be affected by several changes in sea level (Schlanger, 1963). During the Pleistocene epoch, four or more cycles of sea-level rise and fall occurred. Sea level rises during interglacial periods, and falls during glacial periods. With each drop in sea level, as much as 300 vertical ft of reef and lagoonal sediments were exposed to subaerial weathering and erosion. The subsequent rise in sea level caused an accumulation of new reef and lagoonal sediments over the preceding erosional unconformity. These fluctuations affected carbonate depositional sequences on oceanic islands worldwide.

\section{Geohydrologic Units}

The processes that form atolls also produce variations in lithology that affect the occurrence and flow of fresh ground water. In general, fine-grained unconsolidated sediments are deposited on the lagoonside of atoll islands and coarser materials are deposited on the oceanside. The fine-grained and well-sorted lagoonal deposits retard the movement of ground water and restrict mixing with saltwater. In contrast, the coarse and poorly sorted materials on the oceanside of an atoll island facilitate both the flow of ground water and the mixing of fresh ground water with saltwater.

The general geohydrologic characteristics of atoll islands has been described by Ayers and Vacher (1986). The geohydrologic framework of an atoll island can be considered to consist of three units (fig. 5A). The first unit is the reef flat which forms a stable foundation upon which the island's surficial sediments accumulate. This unit thins from the reef front lagoonward pinching out at some distance beneath the island. The permeability of the reef flat is less than that of the underlying unconsolidated sediments. Where the reef flat extends beneath the island, it acts as a confining layer impeding recharge.

The second unit consists of unconsolidated deposits of Holocene age. These deposits occur beneath the soil zone and are composed of silt- to gravel-sized fragments of foraminifera, Halimeda, and coral. Layering and lateral gradation of these deposits affect the occurrence and flow of fresh ground water. Analysis of ground-water tidal fluctuations and grain-size distributions indicate a general areal variation in permeability across an atoll island: relatively high values for sediments bordering the ocean, and lower values for sediments adjacent to the lagoon (Anthony and others, 1989). The presence of lower permeability sediments adjacent to the lagoon results in a lens of fresh ground water that is thicker on the lagoonside of an atoll island.

The third unit consists of highly permeable deposits of Pleistocene age that underlie the Holocene deposits. The primary skeletal material of the Pleistocene deposits is similar to that of the Holocene deposits. The contact between the Holocene and Pleistocene deposits is typically at depths of 50 to $80 \mathrm{ft}$ below sea level and represents a pronounced permeability contrast in which the Pleistocene deposits are more permeable than the Holocene deposits. The highly permeable Pleistocene deposits at Majuro Atoll in the Marshall Islands were found to contain seawater and thin the zone of transition between freshwater and seawater beneath that part of the freshwater lens that is sufficiently thick to extend down to the Pleistocene deposits (Anthony and others, 1989). Core drilling was not done on the outer islands of Chuuk State; however, the islands probably have undergone about the same depositional and tectonic histories and sea-level changes as other atolls in the westem Pacific. Therefore, similar geohydrologic conditions probably exist in the outer islands of Chuuk State.

\section{Occurrence and Development of Ground Water}

The highly permeable soils that form most atoll islands are readily infiltrated by recharge from rainfall. The island intercepts rainfall; a fraction of the rainfall is lost to evapotranspiration, and the remainder infiltrates 


\section{$\boldsymbol{A}$}

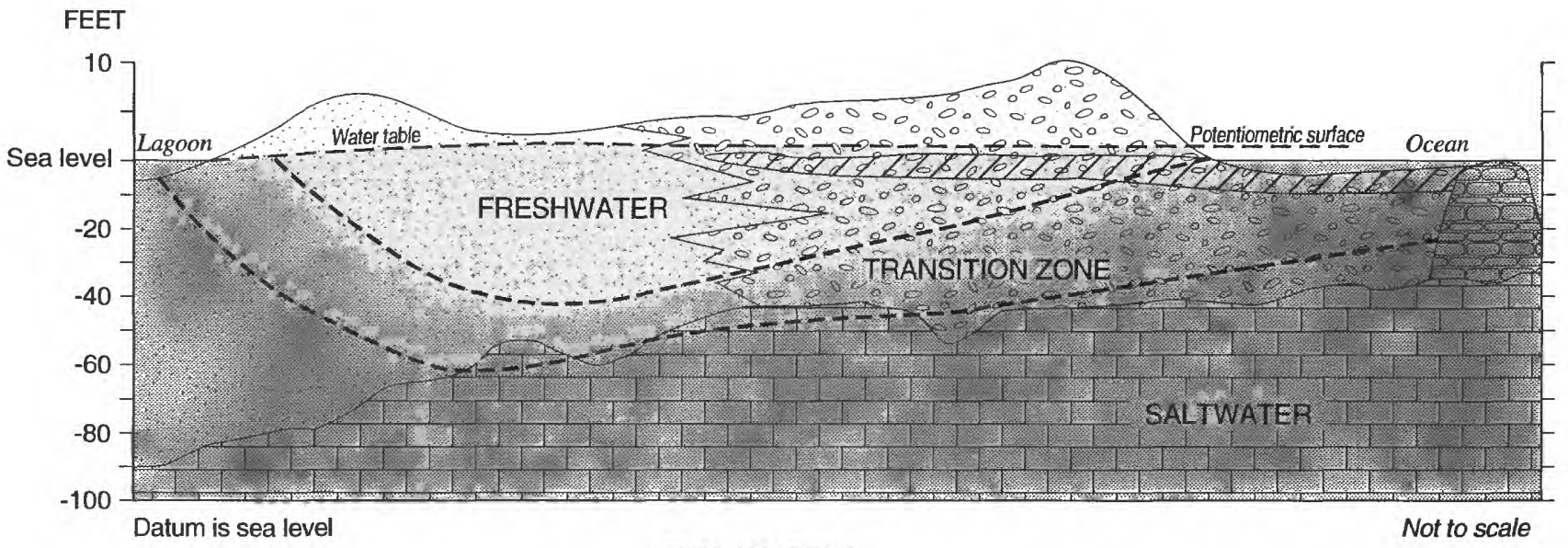

EXPLANATION

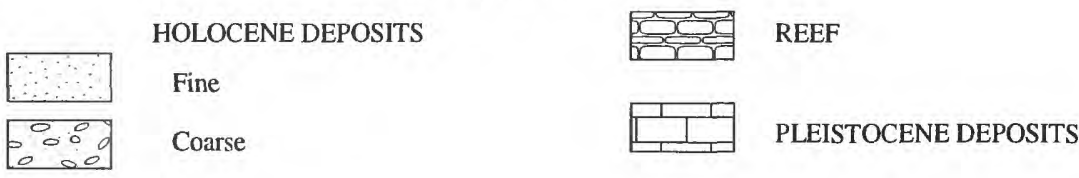

REEF-FLAT PLATE

B

RAINFALL

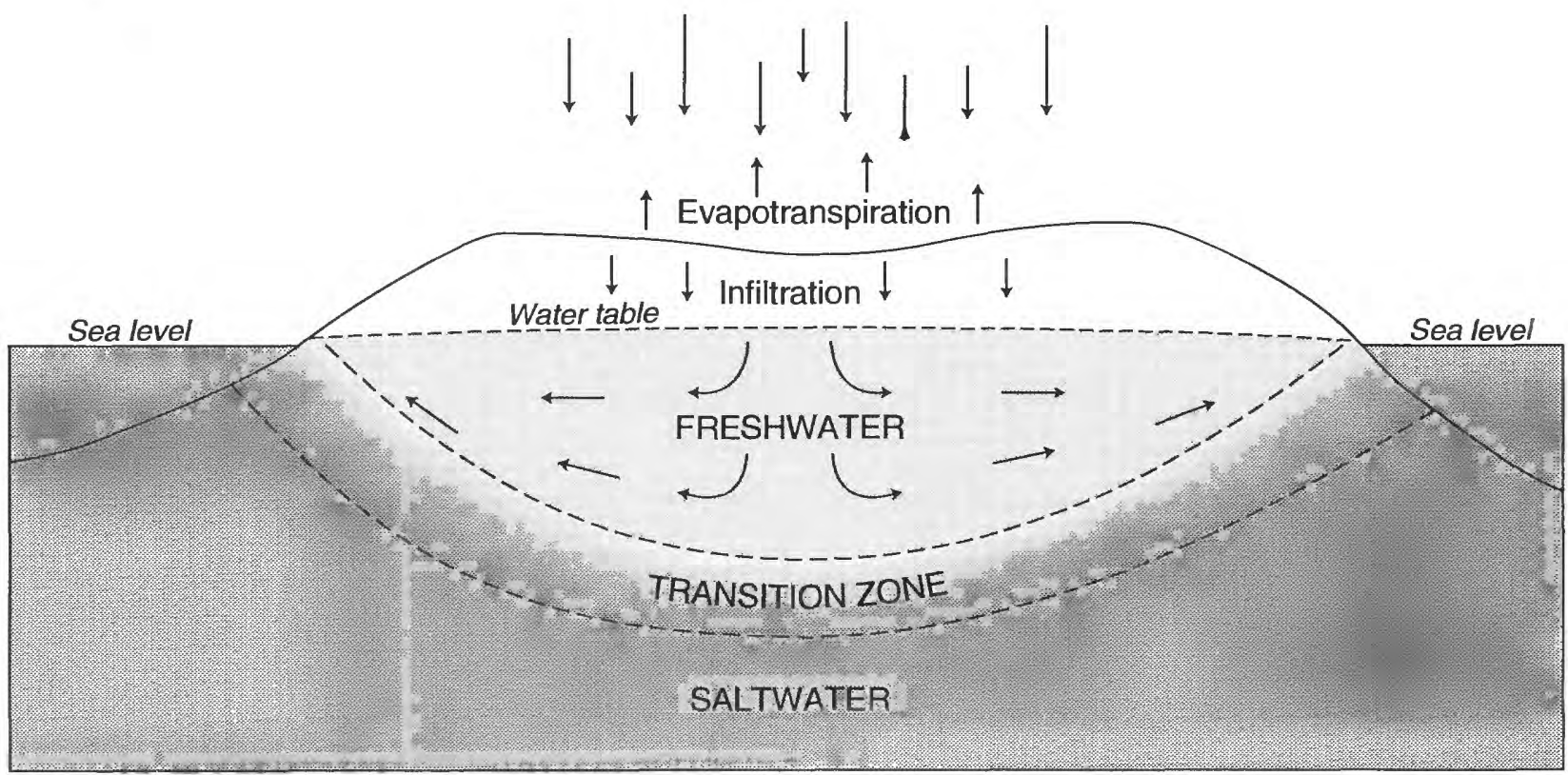

Not to scale

Figure 5. Schematic sections of an atoll island showing $A$, conceptual hydrogeologic framework (modified from Ayers and Vacher, 1986), and $\boldsymbol{B}$, freshwater lens with transition zone (modified from Hamlin and Anthony, 1987). 
the island's sediments. Because of the high permeability of the soils, there is no surface runoff. If the infiltration from rainfall is sufficiently great, a lens-shaped fresh ground-water body forms. The less-dense freshwater floats on the more-dense seawater, somewhat like an iceberg floating in the ocean (fig. 5B). Fresh ground water moves radially outward from the island's center toward the shoreline and discharges to the sea. Some of the freshwater mixes with the underlying seawater to form a zone of transition between freshwater and seawater. As a result of the difference in density between freshwater and seawater, the lens of freshwater extends to a depth of about 40 times the elevation of the lens surface above sea level. This 40:1 ratio is known as the Ghyben-Herzberg relation. The actual thickness of freshwater is influenced by the recharge and discharge rates, the size and shape of the island, and the hydraulic characteristics of the geohydrologic units.

The distribution of fresh ground water in atoll islands primarily is controlled by the size and shape of the island and by variations in the hydraulic characteristics of the geohydrologic units. Large, circular islands generally have large freshwater lenses. Small, elongated islands promote mixing of freshwater with seawater, and are associated with thin freshwater lenses and thick transition zones. The thickest part of the freshwater lens often is found in the fine-grained sediments on the lagoonside of the islands.

Although rainwater is the preferred source for water supply on atoll islands, ground water from shallow dug wells often is used during periods of drought. Historically, ground water was developed for taro cultivation. Taro commonly is cultivated in an elongated interior or central depression between the ocean and lagoonside beach ridges. For the cultivation of wetland taro, the islanders have deepened the central depressions to expose the water table.

During World War II, the Japanese population on the outer islands of Chuuk had a significant effect on the development of water, both in method and quantity (Father Hezel, oral commun., 1984). Between 1934 and 1945 the Japanese population increased from about 25 to about 5,000 , almost doubling the population on the outer islands. The Japanese constructed cisterns to collect rainwater, many of the cisterns were still functional in 1984. With the knowledge that ground water provides a water supply that is less affected by short-term variations in rainfall than rainwater-catchment systems, the Japanese developed ground water for washing dishes and clothes, and to take baths. Many of the dug wells inventoried as part of the reconnaissance survey were constructed by the Japanese during World War II. Most of these wells are lined and curbed with concrete. During droughts and extended dry periods, ground water was and remains the sole source of water supply.

All wells on atoll islands are susceptible to contamination from the surface because of the shallow depth to water (less than $10 \mathrm{ft}$ ) and high permeability of soils. Wells could be constructed to minimize contamination from the surface with a cover or raised concrete platform (minimum lateral extension of $1 \mathrm{ft}$ and minimum height of $1 \mathrm{ft}$ ), and cemented or solid casing (at least $1 \mathrm{ft}$ below the water table). This type of well (fig. 6) could be equipped with a hand or solar pump.

To minimize potential contamination, the wells could be located inland and upgradient of toilets, pig pens, and other sources of pollution. Water in the freshwater lens flows radially outward from a central high area to the surrounding ocean. This high area in the water surface usually is located on the lagoonside of the island, corresponding to the thickest part of the lens and greatest accumulation of fine-grained sediments (Hamlin and Anthony, 1987). If sources of contamination were restricted from this area, wells located here would tap the cleanest and thickest part of the freshwater lens.

\section{WATER QUALITY IN THE INHABITED OUTER ISLANDS}

Major ion data for selected dug wells are shown graphically in a trilinear diagram (fig. 7). Trilinear diagrams display the relative proportions of the most common cations and anions, thereby allowing a comparison of the waters with different total chemical concentrations (Hem, 1985). This type of diagram conveniently shows the effects of mixing water from different sources. Data from samples of ground water from the outer islands of Chuuk State form a straight line on this diagram (fig. 7). This relation indicates simple mixing of fresh ground water with seawater. The end points of this line are sodium-chloride (seawater) and calcium-carbonate (fresh ground water) compositions. Dissolution of carbonate minerals by rainwater produces a calciumcarbonate ground water. Mixing of calcium-carbonate 

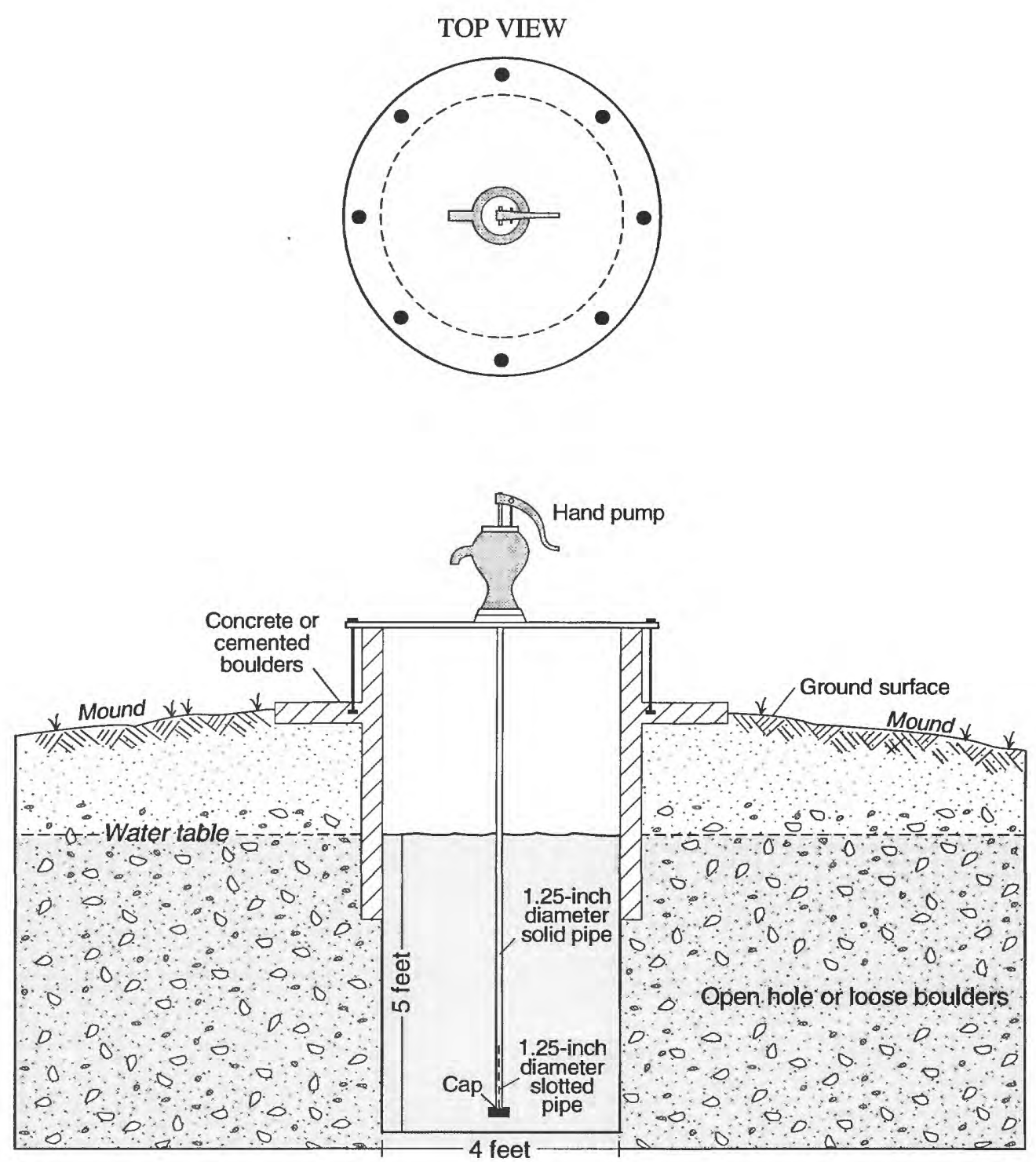

Figure 6. Example of a dug well designed to minimize contamination from surface (modified from Takasaki, 1989).

ground water with seawater yields intermediate compositions of ground water observed in the dug-well waters.

The primary constraint on the use of ground water for water supply in the islands is the chloride concentration. A salty taste becomes noticeable when the chloride concentration exceeds $250 \mathrm{mg} / \mathrm{L}$. As a result, the World Health Organization (WHO) aesthetic quality guideline for chloride is $250 \mathrm{mg} / \mathrm{L}$ (World Health Organization, 1984). Although chloride concentration was not determined for all of the well waters, it can be estimated from the specific conductance data.
The specific conductance of a solution is a measurement of its ability to conduct an electric current and is proportional to the concentration of total dissolved ionic species. As ion concentrations increase, conductance of the solution increases; therefore, the conductance measurement provides an indication of ion concentration (Hem, 1985). The relation between specific conductance and chloride concentration shown in figure 8 may be used to estimate chloride concentration in ground water. Chloride $(\mathrm{Cl})$ and specific conductance (SC) follow the general relation $\mathrm{Cl}=0.32 \mathrm{SC}-205$. 


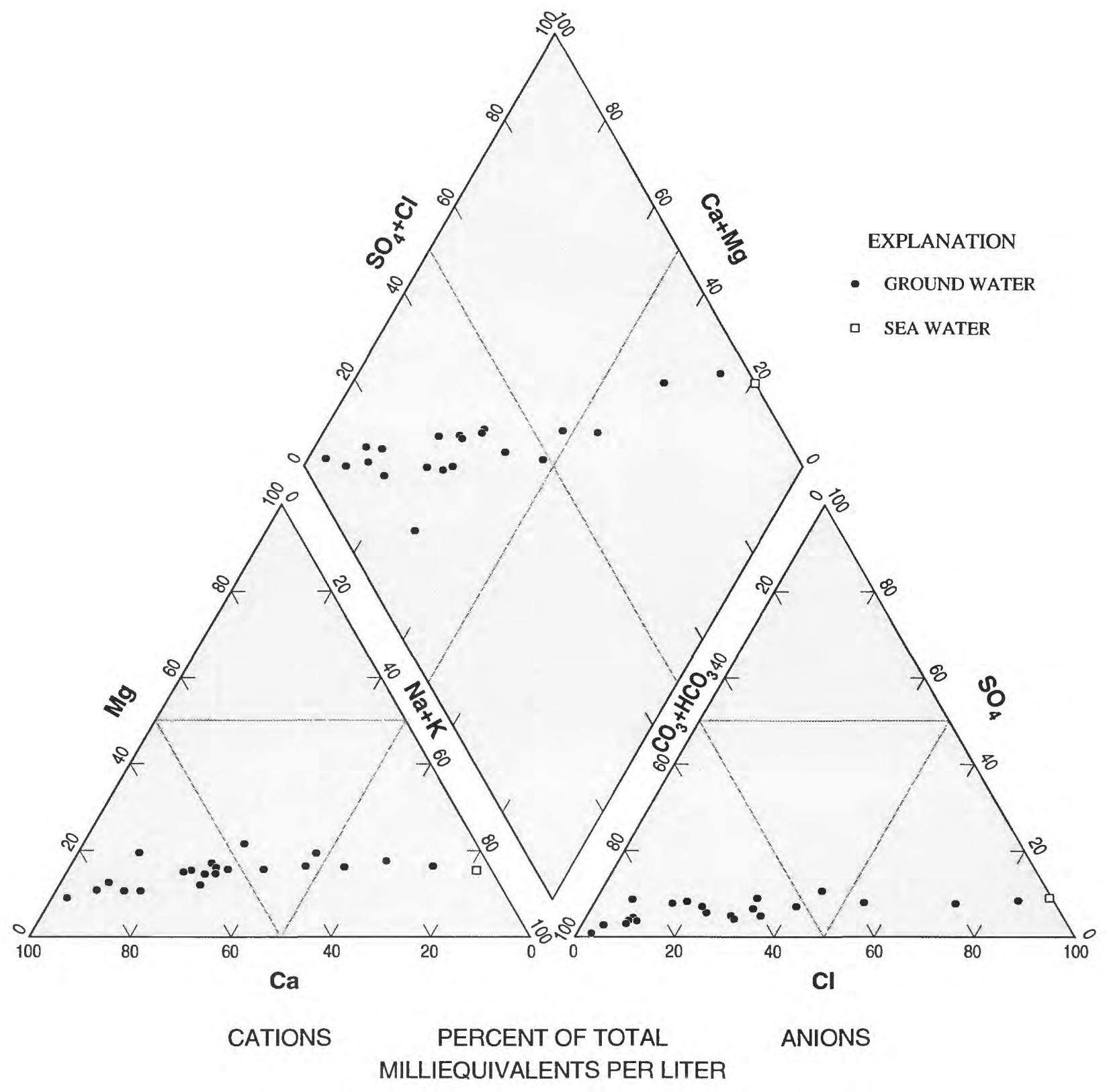

Figure 7. Trilinear diagram showing the range in composition of ground water from dug wells in the inhabited outer islands, Chuuk State, 1984-85. 


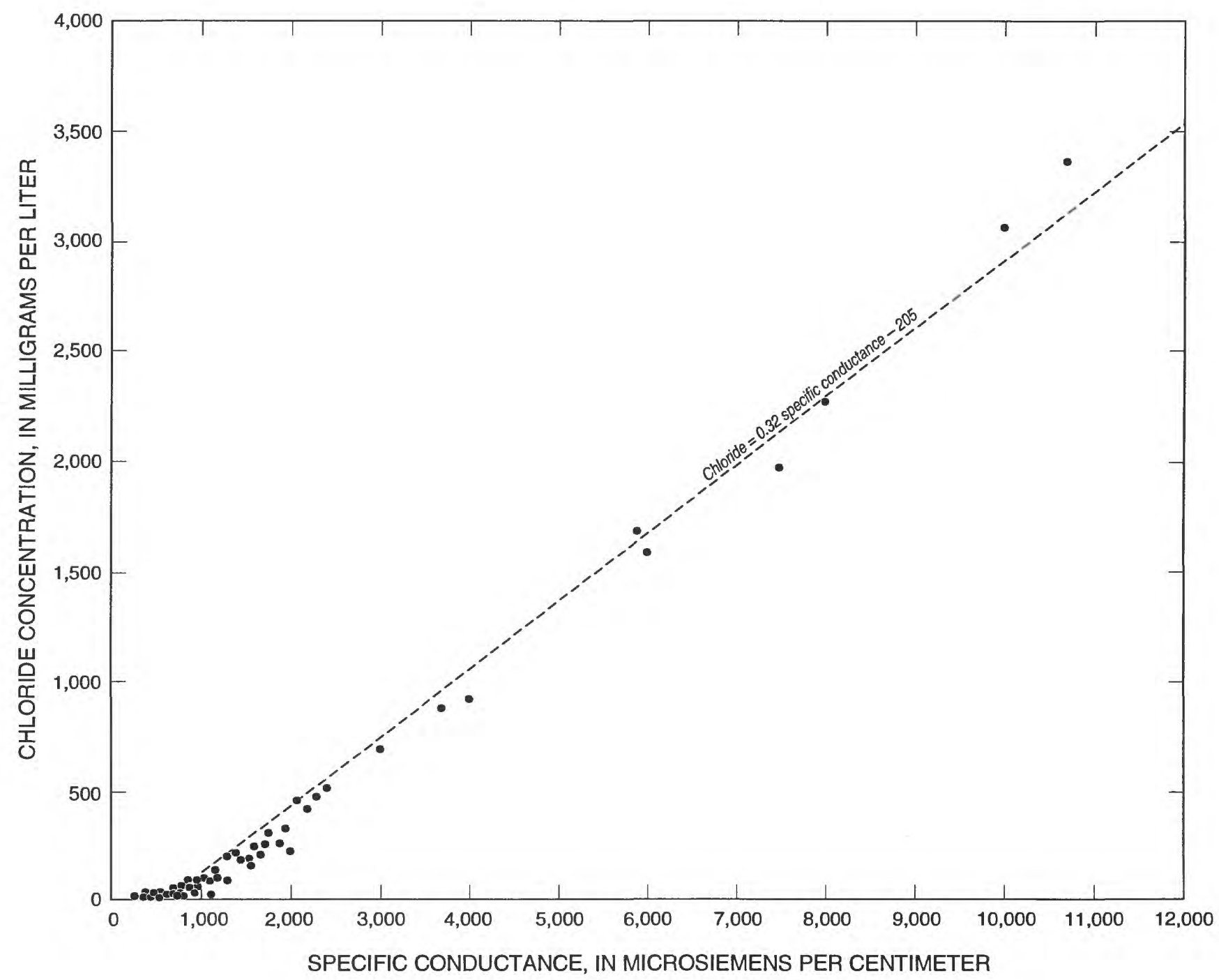

Figure 8. Relation between specific conductance and chloride concentration in ground water from the inhabited outer islands, Chuuk State, 1984-85. 
Values of specific conductance less than $1,500 \mu \mathrm{S} / \mathrm{cm}$ generally are within the guideline for chloride concentration $(250 \mathrm{mg} / \mathrm{L})$ recommended for drinking water by WHO. Water from many of the wells on outer islands in Chuuk State exceed the WHO (1984) guideline for chloride in drinking water. Seawater is the main source of chloride in ground water. Chloride is introduced to ground water from sea spray, wave washover, and seawater intrusion.

One of the most common contaminants in ground water is dissolved nitrogen $(\mathrm{N})$ in the form of nitrate. Excessive nitrate concentrations in drinking water may cause methemoglobinemia in infants. The WHO (1984) guideline for nitrate in drinking water is $10 \mathrm{mg} / \mathrm{L}$ (as N). High levels of nitrate in ground water may indicate contamination from fertilizers or human waste. The presence of nitrate may be associated with pathogenic bacteria from human or animal waste. Natural sources of nitrate in water include atmospheric nitrogen fixation by some plants, plant decay, and animal waste. Nitrate (as $\mathrm{N}$ ) data reported in this report may be converted to nitrate $\left(\right.$ as $\left.\mathrm{NO}_{3}\right)$ using a multiplication factor of 4.4. For example, the safe drinking-water limit of $10 \mathrm{mg} / \mathrm{L}$ (as $\mathrm{N}$ ) is equivalent to a nitrate concentration of $44 \mathrm{mg} / \mathrm{L}$ (as $\mathrm{NO}_{3}$ ).

Trace-element analyses of ground-water samples from selected wells for each island group are listed in tables 1, 6, 12, and 17. All of the measured concentrations of trace elements were within the WHO (1984) guideline for drinking water. Elevated concentrations of trace elements in some of the water samples are most likely related to the casing and to other foreign materials placed in the well. Many of the dug wells were cased with 55-gal oil drums made of metal. Some of the metals that were detected in well water may have been derived from corrosion or leaching of the 55-gal oil drum casing. 


\section{Western Island Group}

The inhabited islands of the Western Island Group are Pollap and Tamatam at Pollap Atoll, Polowat at Polowat Atoll, and Houk (fig. 9). These islands are about 185 mi west of the Chuuk Island Group. Mean values for specific conductance of ground water on each the islands ranged from $700 \mu \mathrm{S} / \mathrm{cm}$ on Houk to 1,510 $\mu \mathrm{S} / \mathrm{cm}$ on Tamatam. Field determinations for nitrate concentration of ground water ranged from less than 1 $\mathrm{mg} / \mathrm{L}$ (as $\mathrm{N}$ ) at selected sites on each of the islands, to a high of $10 \mathrm{mg} / \mathrm{L}$ (as $\mathrm{N}$ ) on Houk Island. Table 1 includes physical, major-ion and trace-element data for selected wells in the Western Island Group. Specific information for each of the islands is presented below.

Table 1. Physical, major ion, and trace element data for selected wells in the Western Island Group, Chuuk State $\left[{ }^{\circ} \mathrm{C}\right.$, degrees Celsius; $\mu \mathrm{S} / \mathrm{cm}$, microsiemens per centimeter at $25^{\circ} \mathrm{C} ; \mathrm{mg} / \mathrm{L}$, milligrams per liter, $\mu \mathrm{g} / \mathrm{L}$, micrograms per liter; $\mathrm{CaCO}_{3}$, calciumcarbonate; --, not measured; $<$, less than]

\begin{tabular}{|c|c|c|c|c|c|}
\hline Property or constituent & Units & $\begin{array}{c}\text { Pollap } \\
\text { well } 11 \\
02 / 04 / 84\end{array}$ & $\begin{array}{c}\text { Tamatam } \\
\text { well } 2 \\
02 / 03 / 84\end{array}$ & $\begin{array}{c}\text { Polowat } \\
\text { well } 32 \\
01 / 31 / 84\end{array}$ & $\begin{array}{c}\text { Houk } \\
\text { well } 7 \\
02 / 05 / 84\end{array}$ \\
\hline Specific conductance ............ & $\mu \mathrm{S} / \mathrm{cm}$ & 1,150 & 1,600 & 517 & 1,100 \\
\hline $\mathrm{pH} \ldots \ldots \ldots \ldots \ldots \ldots \ldots \ldots \ldots \ldots \ldots \ldots$ & units & 6.7 & 7.2 & 6.4 & 6.9 \\
\hline Temperature $\ldots \ldots \ldots \ldots \ldots \ldots \ldots$ & ${ }^{\circ} \mathrm{C}$ & 27.0 & 27.0 & 27.5 & 27.5 \\
\hline Hardness as $\mathrm{CaCO}_{3}$. . & $\mathrm{mg} / \mathrm{L}$ & 420 & 430 & 300 & 420 \\
\hline Calcium, total as Ca . . . . . . . . . & $\mathrm{mg} / \mathrm{L}$ & 130 & 110 & 110 & 130 \\
\hline Magnesium, total as $\mathrm{Mg} . \ldots \ldots \ldots \ldots$ & $\mathrm{mg} / \mathrm{L}$ & 23 & 38 & 6 & 23.5 \\
\hline Sodium, total as Na. . . . . . . . . . & $\mathrm{mg} / \mathrm{L}$ & 87 & 170 & 4 & 34.2 \\
\hline Potassium, total as $\mathrm{K} \ldots \ldots \ldots \ldots \ldots$ & $\mathrm{mg} / \mathrm{L}$ & 1.5 & 13 & $<0$ & 75.1 \\
\hline Alkalinity, total as $\mathrm{CaCO}_{3} \ldots$ & $\mathrm{mg} / \mathrm{L}$ & 383 & 371 & 313 & 491 \\
\hline Sulfate, dissolved as $\mathrm{SO}_{4} \ldots$ & $\mathrm{mg} / \mathrm{L}$ & 28 & 87 & 3 & 20.5 \\
\hline Chloride, dissolved as $\mathrm{Cl} \ldots \ldots \ldots \ldots$ & $\mathrm{mg} / \mathrm{L}$ & 150 & 250 & 5 & 39.3 \\
\hline Fluoride, total as F . . . . . . . . . . & $\mathrm{mg} / \mathrm{L}$ & 0.2 & 0.2 & 0.2 & 0.3 \\
\hline Silica, dissolved as $\mathrm{SO}_{2} \ldots$ & $\mathrm{mg} / \mathrm{L}$ & 0.4 & 1.6 & 0.25 & 2.5 \\
\hline $\begin{array}{l}\text { Dissolved solids, calculated, } \\
\text { sum of constituents ............. }\end{array}$ & $\mathrm{mg} / \mathrm{L}$ & 652 & - & -- & 677 \\
\hline Aluminum, total as $\mathrm{Al} \ldots \ldots \ldots \ldots$ & $\mathrm{mg} / \mathrm{L}$ & 10 & 30 & 10 & 20 \\
\hline Arsenic, total as As. . . . . . . . . . & $\mu g / L$ & $<1$ & $<1$ & $<1$ & $<1$ \\
\hline Barium, total as $\mathrm{Ba} \ldots \ldots \ldots \ldots \ldots$ & $\mu \mathrm{g} / \mathrm{L}$ & $<100$ & $<100$ & $<100$ & $<100$ \\
\hline Beryllium, total as Be........... & $\mu \mathrm{g} / \mathrm{L}$ & $<10$ & $<10$ & $<10$ & $<10$ \\
\hline Cadmium, total as Cd $\ldots \ldots \ldots \ldots \ldots$ & $\mu \mathrm{g} / \mathrm{L}$ & $<1$ & 3 & $<1$ & $<1$ \\
\hline Chromium, total as $\mathrm{Cr} \ldots \ldots \ldots \ldots$. & $\mu \mathrm{g} / \mathrm{L}$ & 20 & 10 & $<10$ & 10 \\
\hline Cobalt, total as Co ............... & $\mu \mathrm{g} / \mathrm{L}$ & $<1$ & $<1$ & $<1$ & $<1$ \\
\hline Copper, total as $\mathrm{Cu} \ldots \ldots \ldots \ldots \ldots$ & $\mu \mathrm{g} / \mathrm{L}$ & 2 & 5 & 6 & 3 \\
\hline Iron, total as Fe $\ldots \ldots \ldots \ldots \ldots \ldots$ & $\mu \mathrm{g} / \mathrm{L}$ & 530 & 46 & 300 & 26 \\
\hline Iron, dissolved as $\mathrm{Fe} \ldots \ldots \ldots \ldots \ldots$ & $\mu \mathrm{g} / \mathrm{L}$ & 170 & 46 & 14 & 26 \\
\hline Lead, total as $\mathrm{Pb} . \ldots \ldots \ldots \ldots \ldots \ldots$ & $\mu \mathrm{g} / \mathrm{L}$ & $<1$ & $<1$ & 9 & 2 \\
\hline Lithium, total as $\mathrm{Li} \ldots \ldots \ldots \ldots \ldots$ & $\mu \mathrm{g} / \mathrm{L}$ & $<10$ & $<10$ & $<10$ & $<10$ \\
\hline Manganese, total as $\mathrm{Mn} . \ldots \ldots \ldots$. & $\mu \mathrm{g} / \mathrm{L}$ & 21 & 16 & 10 & 27 \\
\hline Manganese, dissolved as $\mathrm{Mn} \ldots \ldots \ldots$ & $\mu \mathrm{g} / \mathrm{L}$ & 21 & 16 & 2 & 27 \\
\hline Mercury, total as $\mathrm{Hg} . \ldots \ldots \ldots \ldots \ldots$ & $\mu \mathrm{g} / \mathrm{L}$ & $<0.1$ & $<0.1$ & $<0.1$ & $<0.1$ \\
\hline Molybdenum, total as Mo. ........... & $\mu \mathrm{g} / \mathrm{L}$ & $<1$ & 1 & $<1$ & $<1$ \\
\hline Nickel, total as $\mathrm{Ni} \ldots \ldots \ldots \ldots \ldots$. & $\mu \mathrm{g} / \mathrm{L}$ & 3 & 7 & 4 & 5 \\
\hline Selenium, total as $\mathrm{Se} . . . \ldots \ldots \ldots$ & $\mu \mathrm{g} / \mathrm{L}$ & $<1$ & $<1$ & $<1$ & $<1$ \\
\hline Silver, total as $\mathrm{Ag} \quad \ldots \ldots \ldots \ldots \ldots \ldots$ & $\mu g / L$ & $<1$ & $<1$ & $<1$ & $<1$ \\
\hline 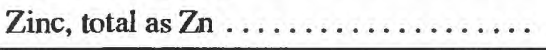 & $\mu \mathrm{g} / \mathrm{L}$ & 30 & 530 & 140 & 20 \\
\hline
\end{tabular}




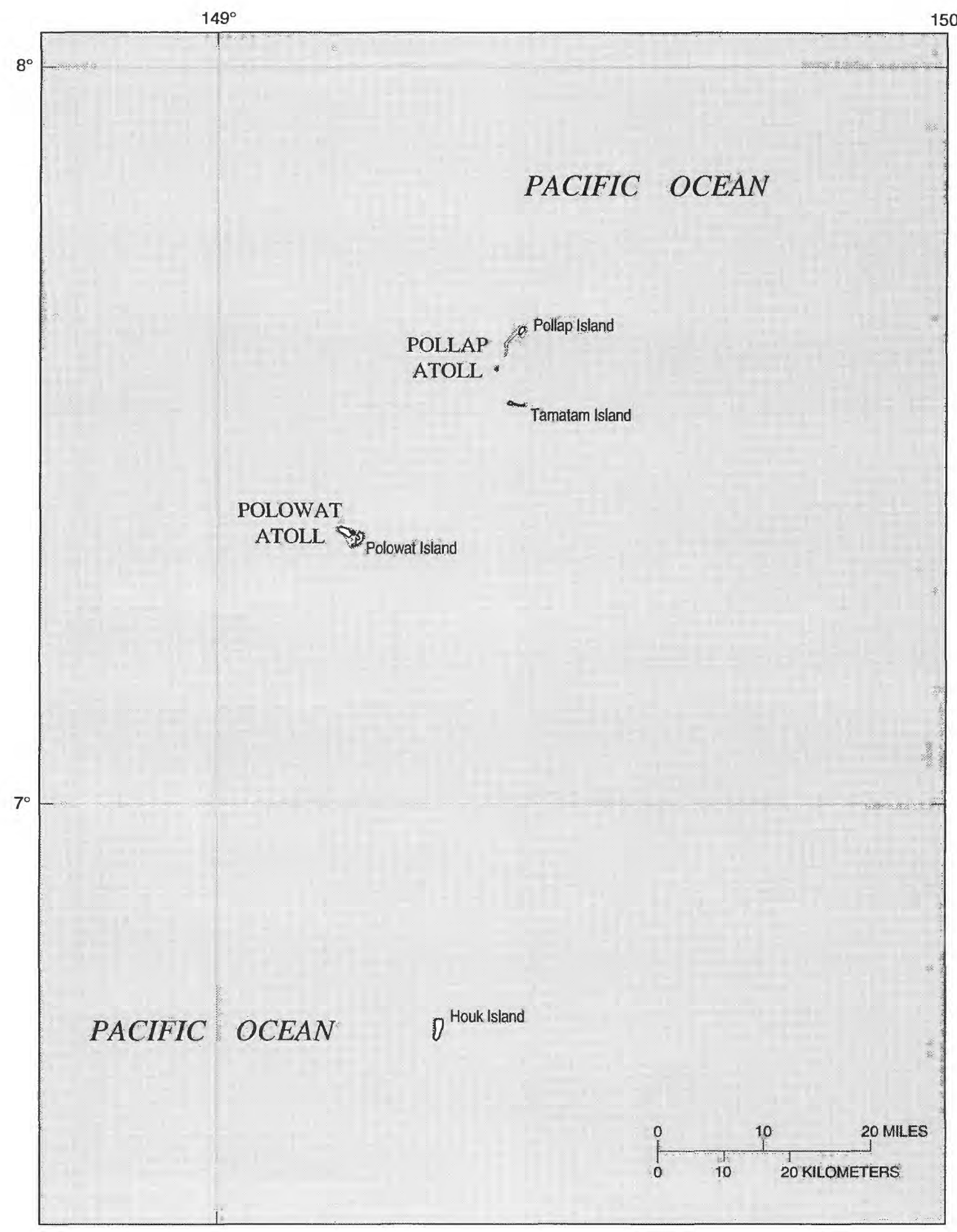

Figure 9. Western Island Group, Chuuk State, Federated States of Micronesia. 


\section{Pollap Island}

The population of Pollap Island at Pollap Atoll is about 432 (Truk State Office of Planning and Statistics, 1981). Well locations, specific conductance, and nitrate concentrations for Pollap Island are shown in figure 10. Selected physical and water-quality data for the $21 \mathrm{dug}$ wells sampled are given in table 2 . The mean specific conductance for water from 21 wells was $1,450 \mu \mathrm{S} / \mathrm{cm}$ and ranged from 370 to $10,000 \mu \mathrm{S} / \mathrm{cm}$. On the basis of the estimated chloride concentrations from specific conductance data, water from 3 of the 21 wells sampled (wells 2, 7, and 14) exceeded the WHO (1984) guideline for chloride. Seven of the 21 wells sampled were field tested for nitrate concentration. Water from 6 of the 7 wells tested had nitrate concentrations of less than $1 \mathrm{mg} / \mathrm{L}$ (as N). Water from well 13 had nitrate concentrations of $5 \mathrm{mg} / \mathrm{L}$ (as $\mathrm{N}$ ).

Table 2. Selected physical and water-quality data from dug wells, February 4 and 6, 1984, Pollap Island, Pollap Atoll, Western Island Group

$\left[{ }^{\circ} \mathrm{C}\right.$, degrees Celsius; $\mu \mathrm{S} / \mathrm{cm}$, microsiemens per centimeter at $25^{\circ} \mathrm{C} ; \mathrm{Cl}$, chloride; $\mathrm{NO}_{3}$, nitrate; $\mathrm{mg} / \mathrm{L}$, milligrams per liter, --, not measured; <, less than]

\begin{tabular}{|c|c|c|c|c|c|c|c|}
\hline $\begin{array}{l}\text { Well } \\
\text { no. }\end{array}$ & Owner & $\begin{array}{c}\text { Temperature } \\
\left({ }^{\circ} \mathrm{C}\right)\end{array}$ & $\begin{array}{c}\text { Specific } \\
\text { conductance } \\
(\mu S / \mathrm{cm})\end{array}$ & pH & $\underset{(\mathrm{mg} / \mathrm{L})}{\mathrm{Cl}}$ & $\begin{array}{c}\mathrm{NO}_{3}, \\
\text { as N } \\
(\mathrm{mg} / \mathrm{L})\end{array}$ & Remarks \\
\hline 1 & Simon & 26.5 & 890 & 6.8 & - & $<1$ & \\
\hline 2 & Kuto & 26.5 & 2,300 & - & - & - & \\
\hline 3 & Seles & 26 & 1,350 & 7.1 & -- & -- & \\
\hline 4 & Marasel & 27 & 1,050 & 6.5 & - & $<1$ & \\
\hline 5 & Roman & 26.5 & 800 & 6.7 & - & $<1$ & \\
\hline 6 & Francisca & 27 & 1,000 & 6.6 & - & -- & \\
\hline 7 & Titus & -- & 10,000 & -- & 3,100 & -- & \\
\hline 8 & Bernard & 26.5 & 370 & 6.9 & 14 & -- & \\
\hline 9 & Oscar & 27 & 650 & 6.7 & - & $<1$ & \\
\hline 10 & Fapian & 28 & 610 & 6.8 & - & -- & \\
\hline 11 & Fermin & 27 & 1,150 & 6.7 & 150 & $<1$ & Major ion and trace element sample. \\
\hline 12 & Sio & 27.5 & 520 & 6.9 & - & -- & \\
\hline 13 & Sixto & 27.5 & 890 & 6.8 & - & 5 & \\
\hline 14 & Erman & 28 & 1,900 & 6.7 & -- & -- & \\
\hline 15 & Bius & 27.5 & 1,500 & 6.8 & -- & $<1$ & \\
\hline 16 & Asisi & 27 & 1,300 & 6.9 & - & - & \\
\hline 17 & Akape & 27.5 & 900 & 6.7 & -- & - & \\
\hline 18 & Sibas & 27.5 & 950 & -- & - & -- & \\
\hline 19 & Lucio & 27 & 850 & -- & - & -- & \\
\hline 20 & Akin & 29 & 550 & - & - & -- & \\
\hline 21 & Marasel & 26.5 & 925 & - & - & -- & \\
\hline
\end{tabular}



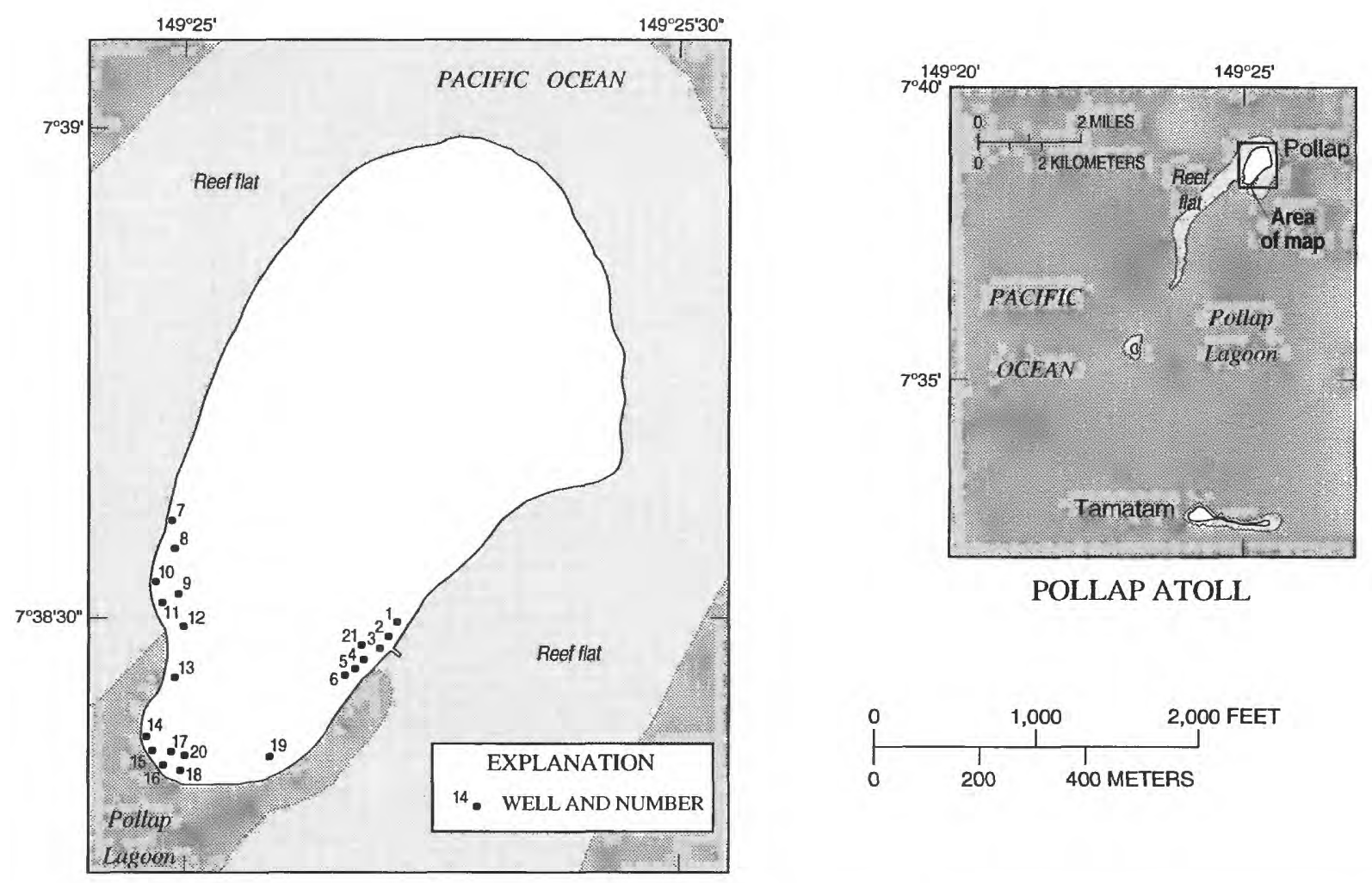

POLLAP ATOLL
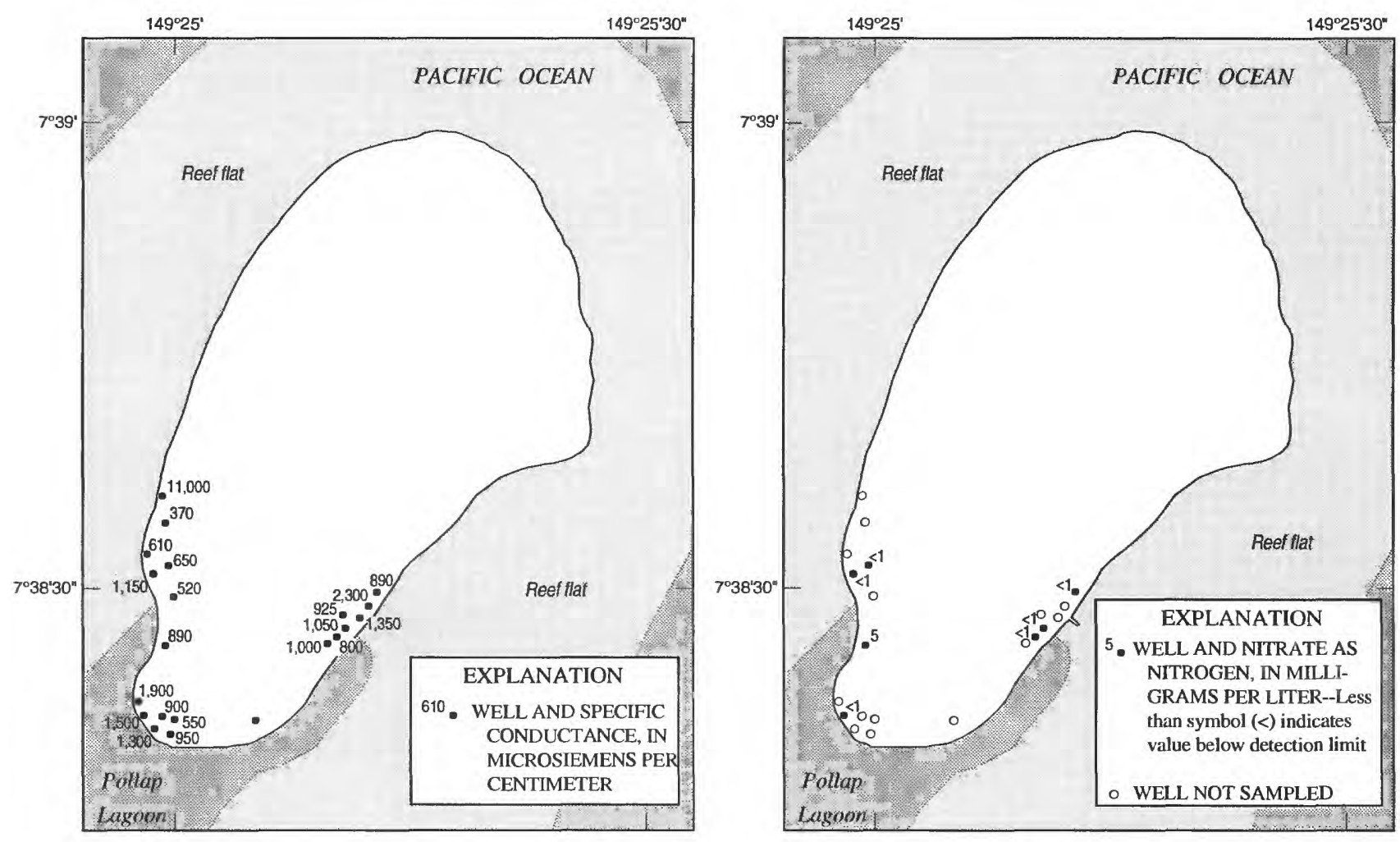

Figure 10. Well locations, specific conductance, and nitrate concentrations for Pollap Island, Pollap Atoll, Western Island Group, Chuuk State, 1984. 


\section{Tamatam Island}

The population of Tamatam Island, Pollap Atoll is about 188 (Truk State Office of Planning and Statistics, 1981). Well locations, specific conductance, and nitrate concentrations for Tamatam Island are shown in figure 11. Selected physical and water-quality data for the 5 dug wells sampled are given in table 3 . The mean specific conductance for water from 5 wells was 1,510 $\mu \mathrm{S} / \mathrm{cm}$ and ranged from 700 to $2,400 \mu \mathrm{S} / \mathrm{cm}$. On the basis of the estimated chloride concentrations from specific conductance data, water from 3 of the 5 wells sampled (wells 2, 3, and 5) exceeded the WHO (1984) guideline for chloride. Four of the 5 wells sampled were field tested for nitrate concentration. Water from 2 of the 5 wells tested had nitrate concentrations of less than $1 \mathrm{mg} / \mathrm{L}$ (as $\mathrm{N}$ ); the other two had concentrations of 5 and $7 \mathrm{mg} / \mathrm{L}$ (as N).

Table 3. Selected physical and water-quality data from dug wells, February 3, 1984, Tamatam Island, Pollap Atoll, Western Island Group

$\left[{ }^{\circ} \mathrm{C}\right.$, degrees Celsius; $\mu \mathrm{S} / \mathrm{cm}$, microsiemens per centimeter at $25^{\circ} \mathrm{C} ; \mathrm{Cl}$, chloride; $\mathrm{NO}_{3}$, nitrate; $\mathrm{mg} / \mathrm{L}$, milligrams per liter; --, not measured; <, less than]

\begin{tabular}{|c|c|c|c|c|c|c|c|}
\hline $\begin{array}{l}\text { Well } \\
\text { no. }\end{array}$ & Owner & $\begin{array}{c}\text { Temperature } \\
\left.\text { ( }{ }^{\circ} \mathrm{C}\right)\end{array}$ & $\begin{array}{c}\text { Specific } \\
\text { conductance } \\
(\mu \mathrm{S} / \mathrm{cm})\end{array}$ & $\mathbf{p H}$ & $\underset{(\mathrm{mg} / \mathrm{L})}{\mathrm{Cl}}$ & $\begin{array}{c}\mathrm{NO}_{3}, \\
\text { as N } \\
\text { (mg/L) }\end{array}$ & Remarks \\
\hline 1 & Rosanto & 26.5 & 700 & 7.3 & 60 & $<1$ & \\
\hline 2 & Robert & 27 & 1,600 & 7.2 & 240 & 7 & Major ion and trace element sample. \\
\hline 3 & Kandito & 27 & 2,400 & 7.0 & 530 & $<1$ & \\
\hline 4 & not known & 27 & 755 & - & 470 & - & \\
\hline 5 & not known & 27.5 & 2,100 & 7.1 & -- & 5 & \\
\hline
\end{tabular}




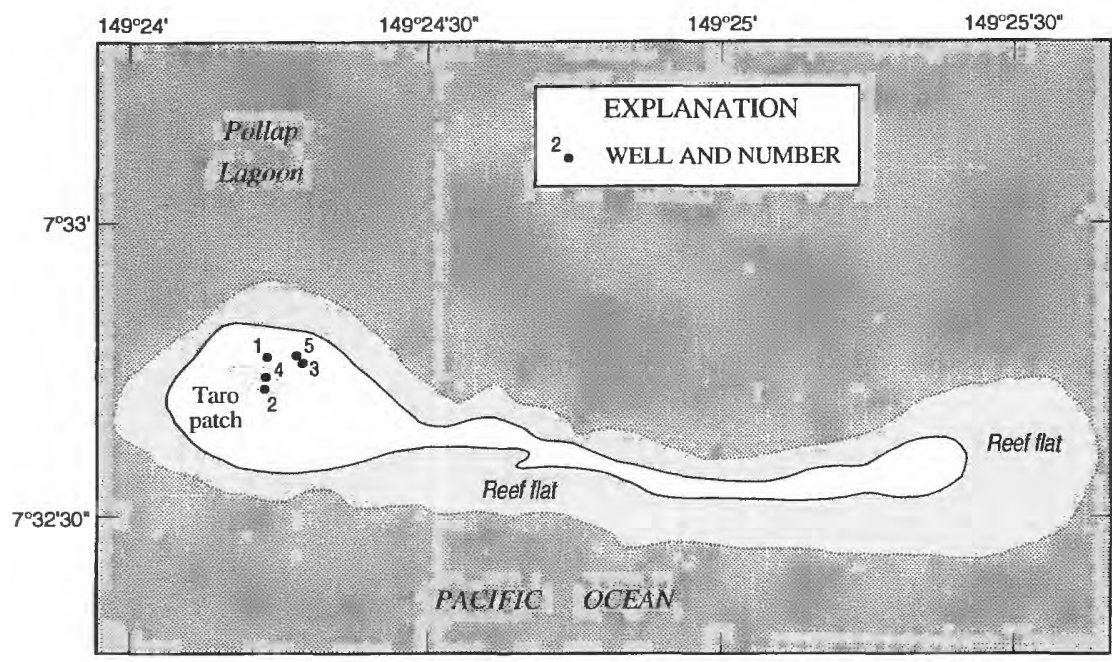

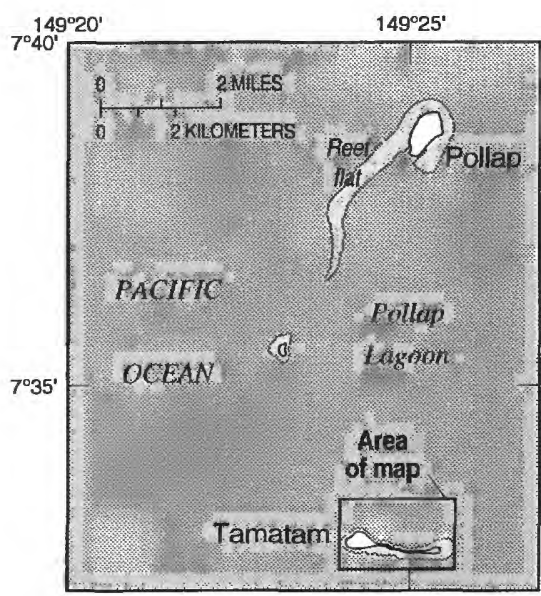

POLLAP ATOLL
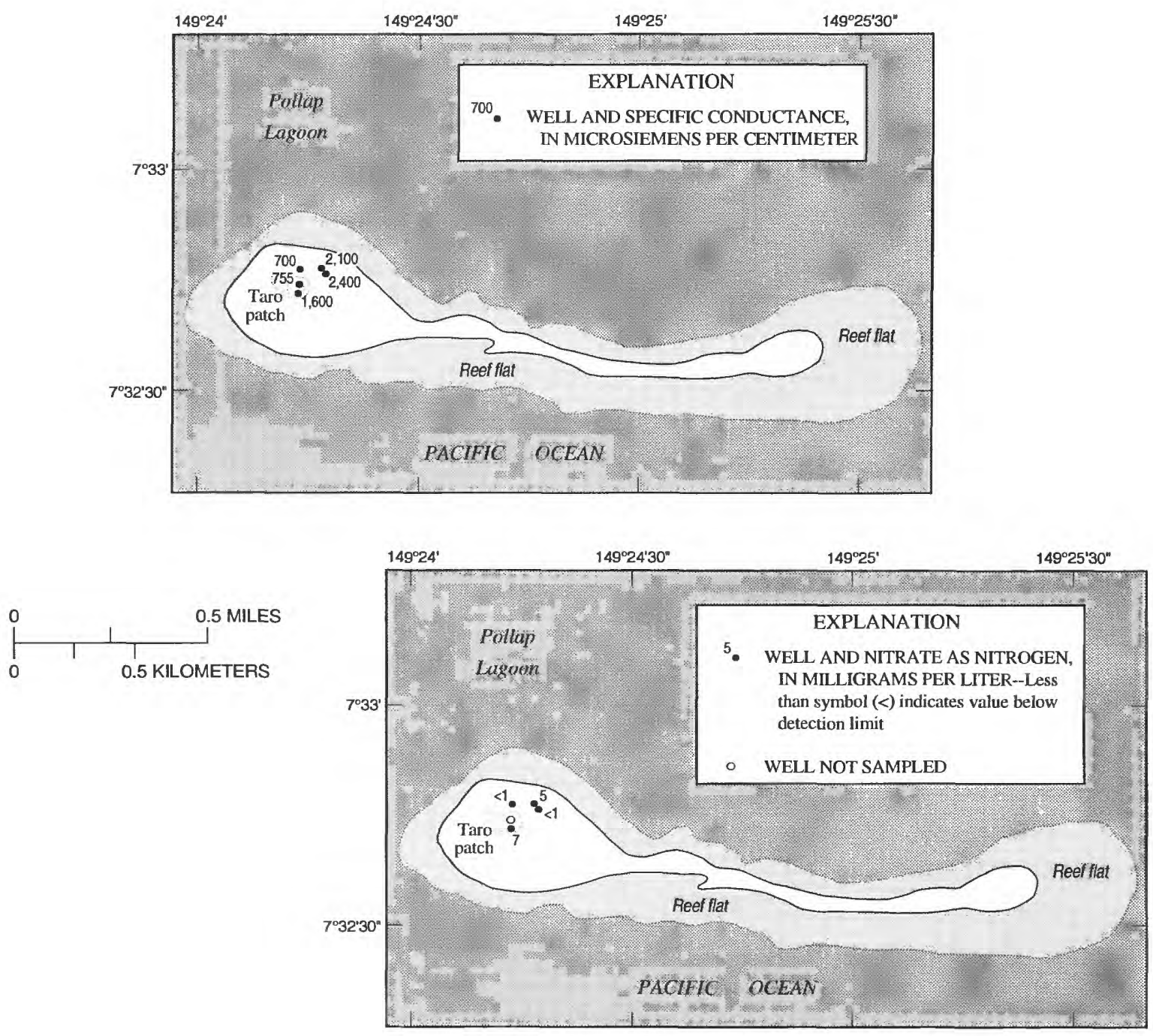

Figure 11. Well locations, specific conductance, and nitrate concentrations for Tamatam Island, Pollap Atoll, Western Island Group, Chuuk State, 1984. 


\section{Polowat Island}

The population of Polowat Island, Polowat Atoll is about 495 ('Truk State Office of Planning and Statistics, 1981). Well locations, specific conductance, and nitrate concentrations for Polowat Island are shown in figure 12. Selected physical and water-quality data for the 31 dug wells, 1 driven well, and 1 taro patch site sampled are given in table 4 . The mean specific conductance for water from 32 wells was $790 \mu \mathrm{S} / \mathrm{cm}$ and ranged from 270 to $1,500 \mu \mathrm{S} / \mathrm{cm}$. On the basis of the estimated chloride concentrations from specific conductance data, none of the well waters sampled exceeded the WHO (1984) guideline for chloride. Five of the 32 wells and the 1 taro patch site sampled were field tested for nitrate concentration. Water from all 5 wells and the 1 taro patch site had nitrate concentrations of less than $1 \mathrm{mg} / \mathrm{L}$ (as N).

Table 4. Selected physical and water-quality data from dug and driven wells and taro patch, January 30-31, 1984, Polowat Island, Polowat Atoll, Western Island Group

[ ${ }^{\circ} \mathrm{C}$, degrees Celsius; $\mu \mathrm{S} / \mathrm{cm}$, microsiemens per centimeter at $25^{\circ} \mathrm{C} ; \mathrm{Cl}$, chloride; $\mathrm{NO}_{3}$, nitrate; $\mathrm{mg} / \mathrm{L}$, milligrams per liter, -, not measured; <, less than]

\begin{tabular}{|c|c|c|c|c|c|c|c|}
\hline $\begin{array}{l}\text { Well } \\
\text { no. }\end{array}$ & Owner & $\begin{array}{c}\text { Temperature } \\
\left({ }^{\circ} \mathrm{C}\right)\end{array}$ & $\begin{array}{c}\text { Speclfic } \\
\text { conductance } \\
(\mu \mathrm{S} / \mathrm{cm})\end{array}$ & pH & $\underset{(\mathrm{mg} / \mathrm{L})}{\mathrm{Cl}}$ & $\begin{array}{c}\mathrm{NO}_{33} \\
\text { as N } \\
(\mathrm{mg} / \mathrm{L})\end{array}$ & Remarks \\
\hline 1 & Lodis-Masair & 26 & 700 & 7.2 & - & $<1$ & \\
\hline 2 & Tilmuar & 26 & 1,350 & 7.1 & - & - & \\
\hline 3 & Bisaln & 27 & 650 & 7.1 & - & - & \\
\hline 4 & Ango & 27 & 725 & 7.3 & - & -- & \\
\hline 5 & Aluka & 26 & 720 & 7.3 & -. & - & \\
\hline 6 & Aisou & 25 & 775 & 6.9 & - & - & \\
\hline 7 & Angkel & 25 & 950 & 7.2 & -- & - & \\
\hline 8 & Tidios & 26 & 520 & 7.3 & -- & - & \\
\hline 9 & Rabuy & 26 & 565 & - & - & - & \\
\hline 10 & Tiduis & 26.5 & 775 & - & - & - & \\
\hline 11 & Soim & 25.5 & 560 & -- & 16 & - & \\
\hline 12 & Rosin & 25 & 580 & -- & - & - & \\
\hline 13 & Ochoman & 26.5 & 620 & - & $\ldots$ & - & \\
\hline 14 & Yoichi & 26.5 & 860 & -- & - & - & \\
\hline 15 & Serepei & 26 & 270 & -- & 24 & $<1$ & \\
\hline 16 & Fapian & 26.5 & 1,500 & - & 200 & - & \\
\hline 17 & Lasin & 26 & 940 & -- & -. & - & \\
\hline 18 & Libokun & 26.5 & 900 & -- & -- & $<1$ & \\
\hline 19 & Iper & 25.5 & 820 & - & - & - & \\
\hline 20 & Urupak & 26 & 580 & - & - & $<1$ & \\
\hline 21 & Pitin & 26 & 850 & -- & - & - & \\
\hline 22 & Aitel & 26 & 1,000 & -- & - & - & \\
\hline 23 & Sour & 27 & 1,450 & -- & - & - & \\
\hline 24 & Yokichi & 27 & 675 & -- & -- & - & \\
\hline 25 & Masmino & 26.5 & 600 & -- & - & - & \\
\hline 26 & Pieiluk & 26.5 & 765 & -- & - & - & \\
\hline 27 & Anasy & 27 & 1,075 & - & - & $<1$ & \\
\hline 28 & Malipy & 28 & 700 & -- & - & - & \\
\hline 29 & Isaocy & 27 & 650 & -. & 19 & - & \\
\hline 30 & Engi & 27 & 1,100 & -- & - & - & \\
\hline 31 & not known & 27 & 500 & -- & - & - & \\
\hline 32 & School & 27.5 & 570 & 6.4 & 6 & -- & Major ion and trace element sample. \\
\hline \multicolumn{2}{|c|}{ Taro patch } & 27.7 & 3,500 & 7.3 & - & $<1$ & \\
\hline
\end{tabular}



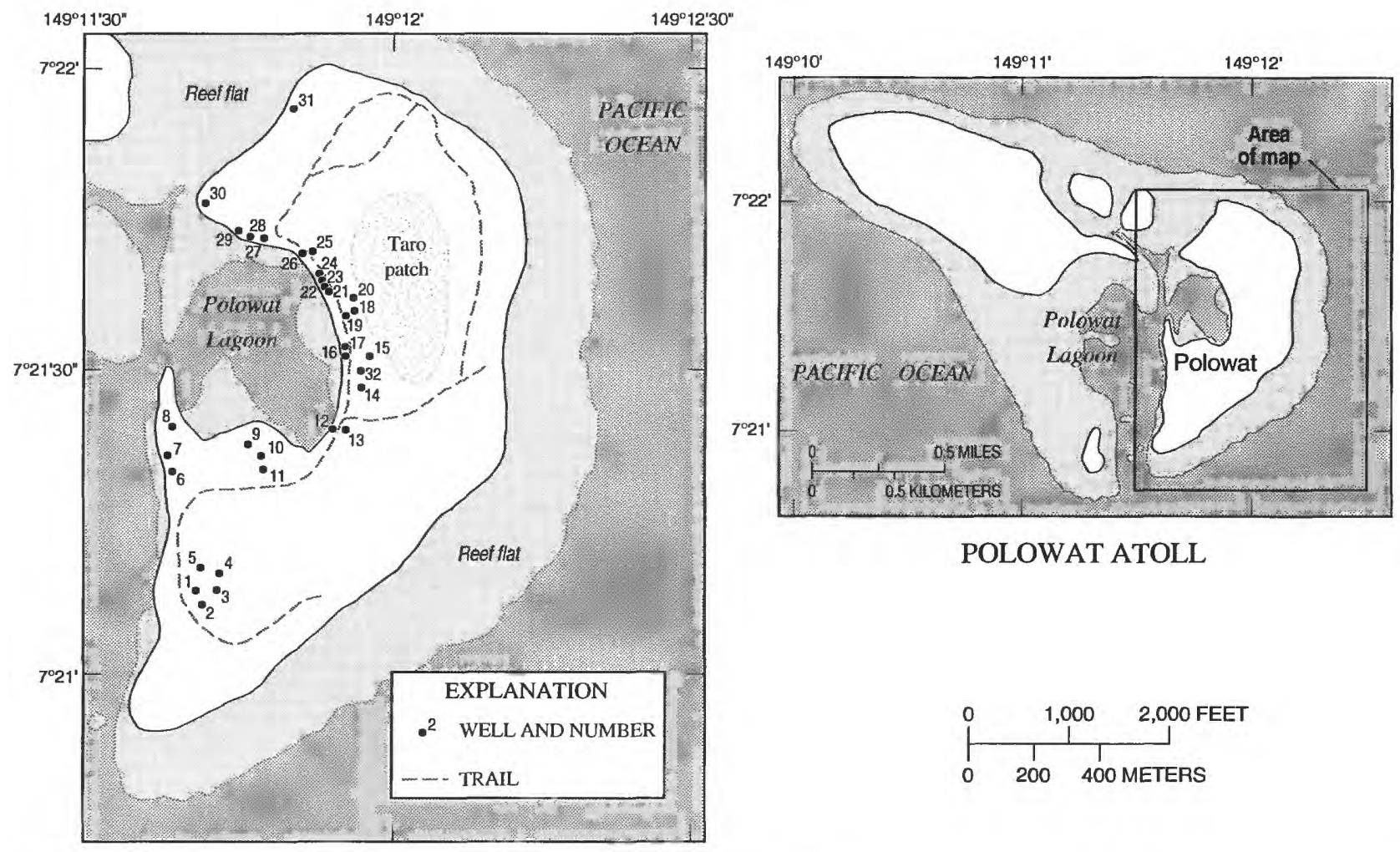

POLOWAT ATOLL
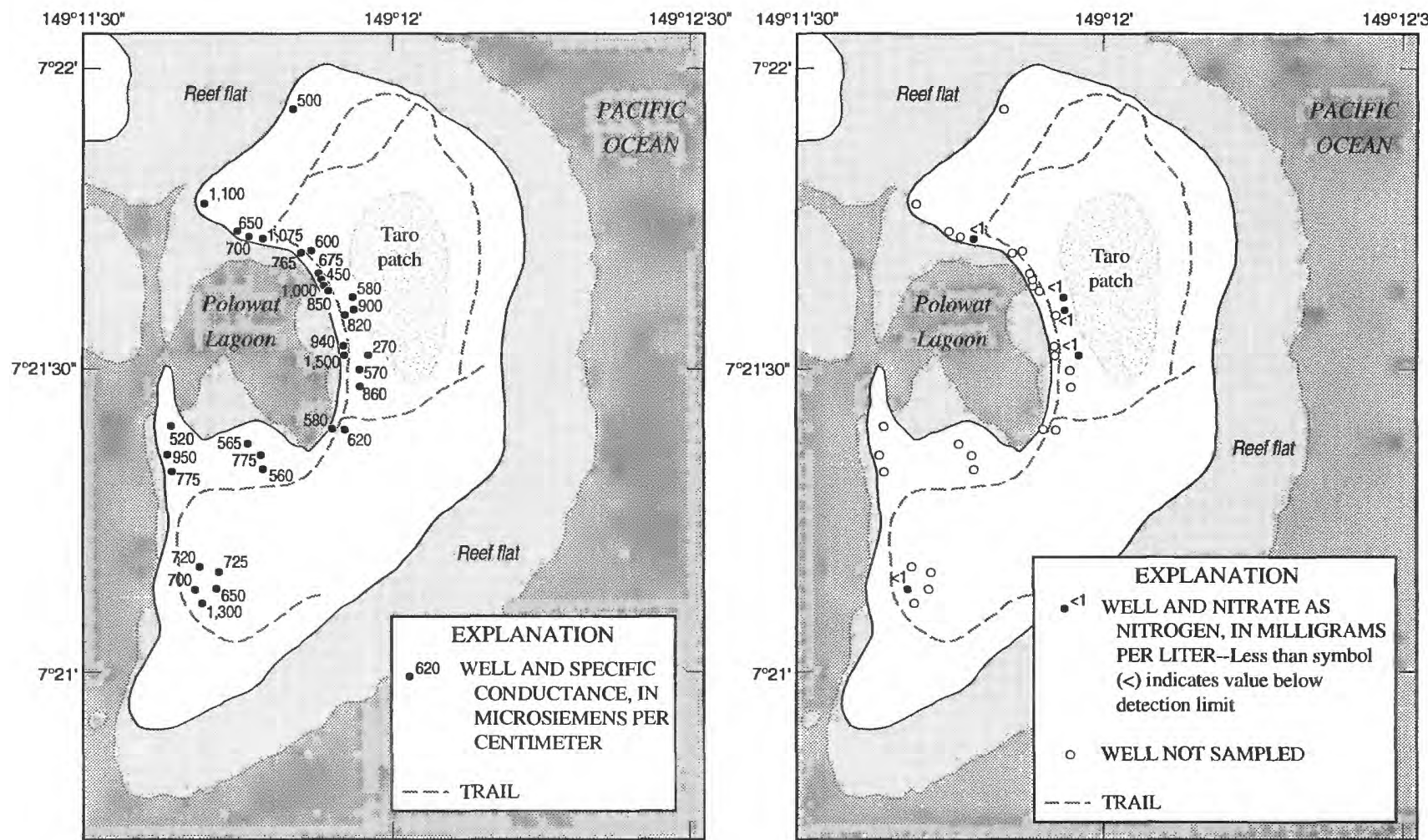

Figure 12. Well locations, specific conductance, and nitrate concentrations for Polowat Island, Polowat Atoll, Western Island Group, Chuuk State, 1984. 


\section{Houk Island}

The population of Houk Island is about 214 (Truk State Office of Planning and Statistics, 1981). Well locations, miscellaneous sampling sites, specific conductance, and nitrate concentrations for Houk Island are shown in figure 13 . Selected physical and water-quality data for the 7 dug wells and 2 miscellaneous sites sampled are given in table 5 . The mean specific conductance for water from 7 wells was $700 \mu \mathrm{S} / \mathrm{cm}$ and ranged from 390 to $1,100 \mu \mathrm{S} / \mathrm{cm}$. On the basis of the estimated chloride concentrations from specific conductance data, none of the well waters sampled exceeded the WHO (1984) guideline for chloride. The highest measured value of specific conductance was for a water sample collected from Lenom Lagoon $(10,700 \mu \mathrm{S} / \mathrm{cm})$. Five of the 7 wells sampled were field tested for nitrate concentration. Water from 2 of the wells tested had nitrate concentrations of less than $1 \mathrm{mg} / \mathrm{L}$ (as $\mathrm{N}$ ); the others had concentrations of 6,7 , and $10 \mathrm{mg} / \mathrm{L}$ (as N). A laboratory analysis of water from well 7 resulted in a nitrate plus nitrite concentration of $13 \mathrm{mg} / \mathrm{L}$ (as $\mathrm{N}$ ) (table 1), which exceeds the WHO (1984) guideline for nitrate.

Table 5. Selected physical and water-quality data from dug wells and miscellaneous sampling sites, February 5, 1984, Houk Island, Polowat Atoll, Western Island Group

$\left[{ }^{\circ} \mathrm{C}\right.$, degrees Celsius; $\mu \mathrm{S} / \mathrm{cm}$, microsiemens per centimeter at $25^{\circ} \mathrm{C} ; \mathrm{Cl}$, chloride; $\mathrm{NO}_{3}$, nitrate; $\mathrm{mg} / \mathrm{L}$, milligrams per liter, $\cdots$, not measured; $<$, less than]

\begin{tabular}{|c|c|c|c|c|c|c|c|}
\hline $\begin{array}{l}\text { Well no. } \\
\text { or sampling } \\
\text { site }\end{array}$ & Owner & $\begin{array}{c}\text { Temperature } \\
\left({ }^{\circ} \mathrm{C}\right)\end{array}$ & $\begin{array}{c}\text { Specific } \\
\text { conductance } \\
(\mu \mathrm{S} / \mathrm{cm})\end{array}$ & pH & $\underset{(\mathrm{mg} / \mathrm{L})}{\mathrm{Cl}}$ & $\begin{array}{c}\mathrm{NO}_{3}, \\
\text { as } \mathrm{N} \\
(\mathrm{mg} / \mathrm{L})\end{array}$ & Remarks \\
\hline 1 & Lekin & 27 & 1,000 & 7.3 & - & - & \\
\hline 3 & Sitow & 27.5 & 690 & 7.0 & -- & 7 & \\
\hline 4 & Leop & 26.5 & 390 & 7.2 & -- & $<1$ & \\
\hline 7 & Chief Pei-ong & 27.5 & 1,100 & 6.9 & 39 & 10 & Major ion and trace element sample. \\
\hline \multicolumn{2}{|c|}{ Miscellaneous site 1} & 26.5 & 500 & - & - & - & \\
\hline \multicolumn{2}{|c|}{ Miscellaneous site 2} & 28.5 & $10,700^{1}$ & - & 3,400 & - & \\
\hline
\end{tabular}

${ }^{1}$ Laboratory determination 

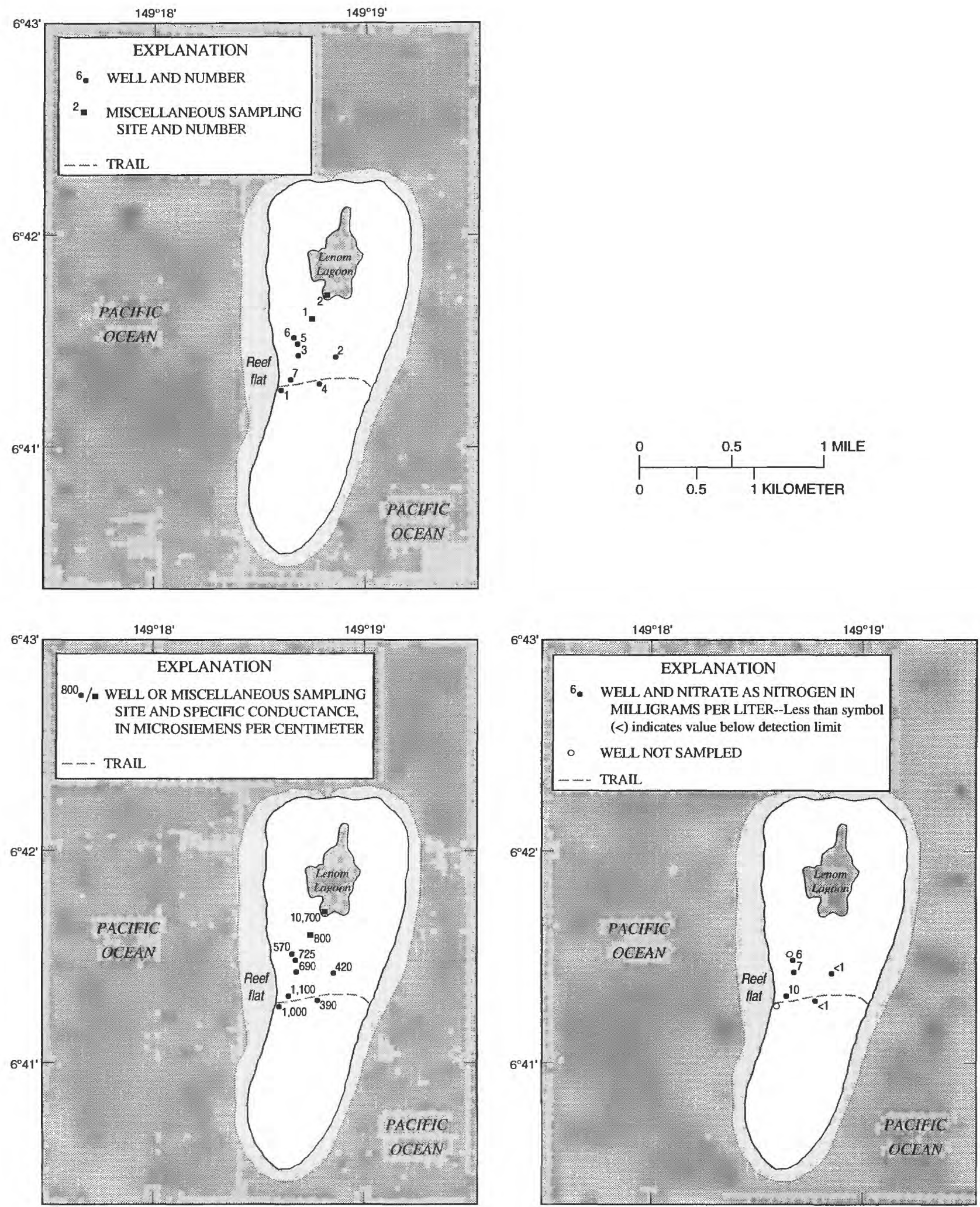

Figure 13. Well locations, specific conductance, and nitrate concentrations for Houk Island, Western Island Group, Chuuk State, 1985. 


\section{Namonweito Island Group}

The inhabited islands of the Namonweito Island Group are Onoun, Makur, Onou, Unanu, and Piherarh Islands at Namonweito Atoll (fig. 14). These islands are about 160 mi northwest of the Chuuk Island Group. Mean values for specific conductance of ground water on each of the islands ranged from $1,070 \mu \mathrm{S} / \mathrm{cm}$ on
Onoun Island to $6,750 \mu \mathrm{S} / \mathrm{cm}$ on Onou Island. Field determinations for nitrate concentration of ground water ranged from less than $1 \mathrm{mg} / \mathrm{L}$ on Onoun and Unanu Islands to $15 \mathrm{mg} / \mathrm{L}$ on Onoun Island. Table 6 includes physical, major-ion, and trace-element data for selected wells in the Namonweito Island Group. Specific information for each of the islands is presented below.

Table 6. Physical, major ion, and trace element data for selected wells in the Piherarh and Onoun Islands, Namonweito Atoll Group, Chuuk State

$\left[{ }^{\circ} \mathrm{C}\right.$, degrees Celsius; $\mu \mathrm{S} / \mathrm{cm}$, microsiemens per centimeter at $25^{\circ} \mathrm{C} ; \mathrm{mg} / \mathrm{L}$, milligrams per liter, $\mu \mathrm{g} / \mathrm{L}$, micrograms per liter, $\mathrm{CaCO}_{3}, \mathrm{Calcium}$-carbonate; $<$, less than]

\begin{tabular}{|c|c|c|c|}
\hline Property or constituent & Units & $\begin{array}{c}\text { Piherarh } \\
\text { well } 5 \\
03 / 21 / 84\end{array}$ & $\begin{array}{c}\text { Onoun } \\
\text { well } 1 \\
03 / 23 / 84\end{array}$ \\
\hline Specific conductance $\ldots \ldots \ldots \ldots \ldots \ldots \ldots \ldots$ & $\mu \mathrm{S} / \mathrm{cm}$ & 950 & 1,750 \\
\hline $\mathrm{pH}, \ldots \ldots \ldots \ldots \ldots \ldots \ldots \ldots \ldots \ldots \ldots \ldots$ & units & 6 & 7.8 \\
\hline Temperature.$\ldots \ldots \ldots \ldots \ldots \ldots \ldots \ldots \ldots$ & ${ }^{\circ} \mathrm{C}$ & 27.5 & 28.5 \\
\hline Hardness as $\mathrm{CaCO}_{3}$ & $\mathrm{mg} / \mathrm{L}$ & 390 & 390 \\
\hline Calcium, total as $\mathrm{Ca} . \ldots \ldots \ldots \ldots \ldots \ldots \ldots$ & $\mathrm{mg} / \mathrm{L}$ & 130 & 100 \\
\hline Magnesium, total as $\mathrm{Mg} . \ldots \ldots \ldots \ldots \ldots \ldots$ & $\mathrm{mg} / \mathrm{L}$ & 15 & 34 \\
\hline Sodium, total as $\mathrm{Na} \ldots \ldots \ldots \ldots \ldots \ldots \ldots$ & $\mathrm{mg} / \mathrm{L}$ & 61 & 200 \\
\hline Potassium, total as $\mathrm{K} \ldots \ldots \ldots \ldots \ldots \ldots \ldots$ & $\mathrm{mg} / \mathrm{L}$ & 13 & 25 \\
\hline Alkalinity, total as $\mathrm{CaCO}_{3} \ldots \ldots \ldots \ldots \ldots$ & $\mathrm{mg} / \mathrm{L}$ & 335 & 330 \\
\hline Sulfate, dissolved as $\mathrm{SO}_{4} \ldots \ldots \ldots \ldots \ldots \ldots$ & $\mathrm{mg} / \mathrm{L}$ & 22 & 65 \\
\hline Chloride, dissolved as $\mathrm{Cl} \ldots \ldots \ldots \ldots \ldots \ldots$ & $\mathrm{mg} / \mathrm{L}$ & 100 & 320 \\
\hline Fluoride, total as $\mathrm{F} \ldots \ldots \ldots \ldots \ldots \ldots \ldots \ldots$ & $\mathrm{mg} / \mathrm{L}$ & 0.2 & 0.4 \\
\hline Silica, dissolved as $\mathrm{SO}_{2} \ldots \ldots$. & $\mathrm{mg} / \mathrm{L}$ & 1.4 & 7.6 \\
\hline $\begin{array}{l}\text { Dissolved solids, calculated, } \\
\text { sum of constituents } \ldots \ldots \ldots \ldots \ldots \ldots \ldots \ldots\end{array}$ & $\mathrm{mg} / \mathrm{L}$ & 597 & 1,020 \\
\hline Aluminum, total as $\mathrm{Al} \ldots \ldots \ldots \ldots \ldots \ldots \ldots$ & $\mathrm{mg} / \mathrm{L}$ & 30 & 30 \\
\hline Arsenic, total as As $\ldots \ldots \ldots \ldots \ldots \ldots \ldots$ & $\mu \mathrm{g} / \mathrm{L}$ & $<1$ & 2 \\
\hline Barium, total as $\mathrm{Ba} . \ldots \ldots \ldots \ldots \ldots \ldots \ldots \ldots$ & $\mu \mathrm{g} / \mathrm{L}$ & $<100$ & $<100$ \\
\hline Beryllium, total as $\mathrm{Be} \ldots \ldots \ldots \ldots \ldots \ldots \ldots$ & $\mu \mathrm{g} / \mathrm{L}$ & $<10$ & $<10$ \\
\hline Cadmium, total as $\mathrm{Cd} . \ldots \ldots \ldots \ldots \ldots \ldots$ & $\mu \mathrm{g} / \mathrm{L}$ & $<1$ & $<1$ \\
\hline Chromium, total as $\mathrm{Cr} \ldots \ldots \ldots \ldots \ldots \ldots \ldots$ & $\mu \mathrm{g} / \mathrm{L}$ & 20 & 20 \\
\hline Cobalt, total as Co $\ldots \ldots \ldots \ldots \ldots \ldots \ldots \ldots$. & $\mu \mathrm{g} / \mathrm{L}$ & $<1$ & $<1$ \\
\hline Copper, total as $\mathrm{Cu} . \ldots \ldots \ldots \ldots \ldots \ldots \ldots$. & $\mu \mathrm{g} / \mathrm{L}$ & 2 & 6 \\
\hline Iron, total as $\mathrm{Fe} \ldots \ldots \ldots \ldots \ldots \ldots \ldots \ldots$ & $\mu \mathrm{g} / \mathrm{L}$ & 19 & 50 \\
\hline Iron, dissolved as $\mathrm{Fe} \ldots \ldots \ldots \ldots \ldots \ldots \ldots$ & $\mu \mathrm{g} / \mathrm{L}$ & 19 & 23 \\
\hline Lead, total as $\mathrm{Pb} . \ldots \ldots \ldots \ldots \ldots \ldots \ldots \ldots$ & $\mu \mathrm{g} / \mathrm{L}$ & 4 & 3 \\
\hline Lithium, total as Li. . . . . . . . . . . . . . . & $\mu \mathrm{g} / \mathrm{L}$ & $<10$ & 10 \\
\hline Manganese, total as Mn ................. & $\mu \mathrm{g} / \mathrm{L}$ & 62 & 65 \\
\hline Manganese, dissolved as $\mathrm{Mn} \ldots \ldots \ldots \ldots \ldots \ldots$ & $\mu \mathrm{g} / \mathrm{L}$ & 62 & 65 \\
\hline Mercury, total as $\mathrm{Hg} \ldots \ldots \ldots \ldots \ldots \ldots \ldots$ & $\mu \mathrm{g} / \mathrm{L}$ & 0.1 & $<0.1$ \\
\hline Molybdenum, total as Mo $\ldots \ldots \ldots \ldots \ldots \ldots \ldots$ & $\mu \mathrm{g} / \mathrm{L}$ & $<1$ & 2 \\
\hline Nickel, total as $\mathrm{Ni} \ldots \ldots \ldots \ldots \ldots \ldots \ldots \ldots$ & $\mu \mathrm{g} / \mathrm{L}$ & $<1$ & 8 \\
\hline Selenium, total as Se $\ldots \ldots \ldots \ldots \ldots \ldots \ldots \ldots$ & $\mu \mathrm{g} / \mathrm{L}$ & $<1$ & $<1$ \\
\hline Silver, total as Ag. . . . . . . . . . . . . . . & $\mu \mathrm{g} / \mathrm{L}$ & $<1$ & $<1$ \\
\hline Zinc, total as $\mathrm{Zn} . \ldots \ldots \ldots \ldots \ldots \ldots \ldots \ldots$ & $\mu \mathrm{g} / \mathrm{L}$ & 10 & 140 \\
\hline
\end{tabular}




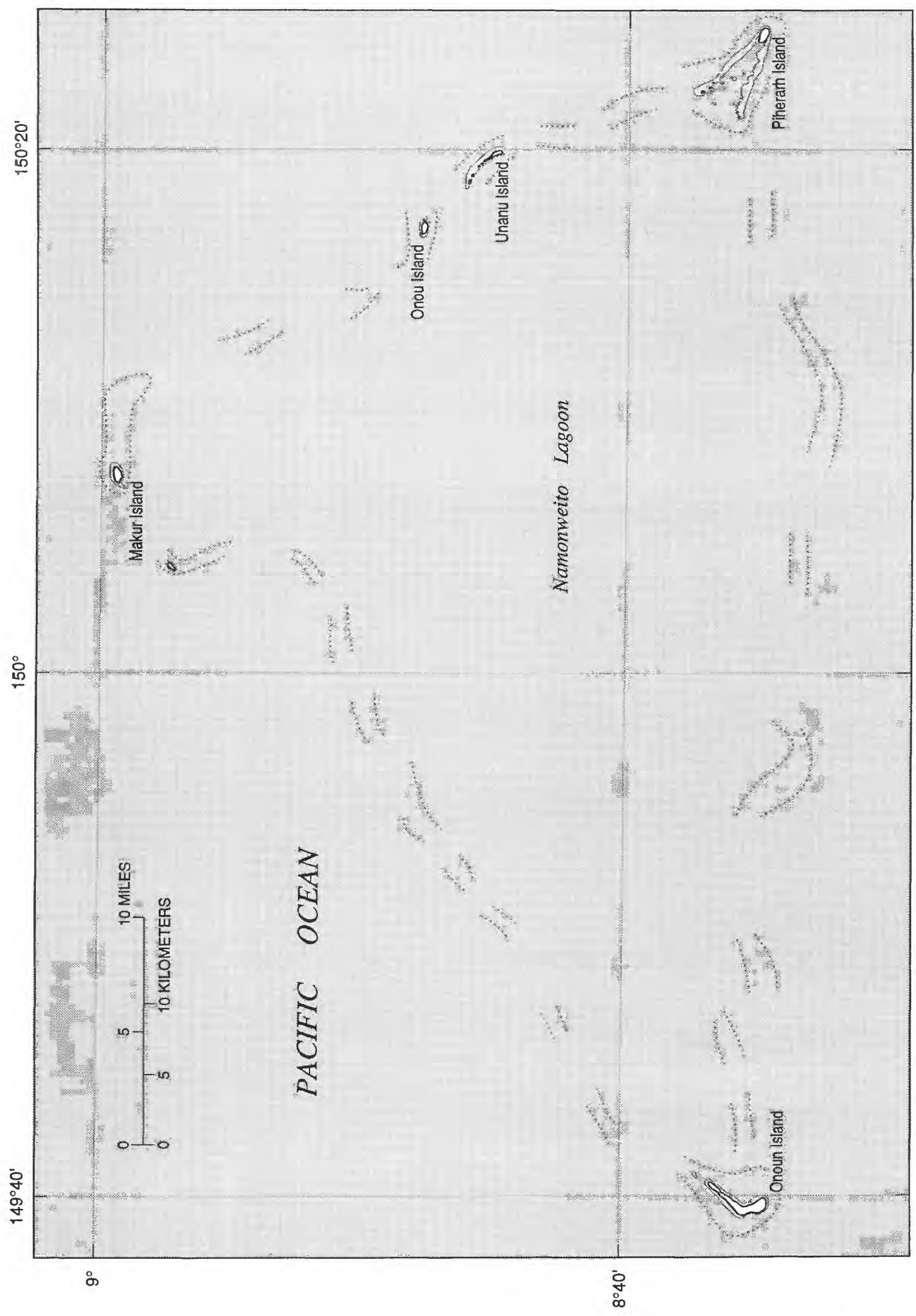

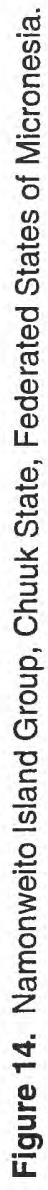




\section{Onoun Island}

The population of Onoun Island, Namonweito Atoll is about 446 (Truk State Office of Planning and Statistics, 1981). Well locations, specific conductance, and nitrate concentrations for Onoun Island are shown in figure 15 . Selected physical and water-quality data for the 41 dug wells and 1 taro patch site sampled are given in table 7 . The mean specific conductance for water from 41 wells was $1,070 \mu \mathrm{S} / \mathrm{cm}$ and ranged from 335 to $1,750 \mu \mathrm{S} / \mathrm{cm}$. On the basis of the estimated chlo- ride concentrations from specific conductance data, water from 6 of the 41 wells sampled (wells $1,3,15,17$, 19 , and 26) exceeded the WHO (1984) guideline for chloride. All 41 of the wells sampled were field tested for nitrate concentration. Water from about half of the wells tested had nitrate concentrations of less than 1 $\mathrm{mg} / \mathrm{L}$ (as N); the remaining well waters had concentrations between 1 and $15 \mathrm{mg} / \mathrm{L}$ (as N). Two of these water samples (wells 1 and 3) exceeded the WHO (1984) guideline for nitrate.

Table 7. Selected physical and water-quality data from dug wells and taro patch site, March 23, 1984, Onoun Island, Namonweito Atoll, Namonweito Island Group

$\left[{ }^{\circ} \mathrm{C}\right.$, degrees Celsius; $\mu \mathrm{S} / \mathrm{cm}$, microsiemens per centimeter at $25^{\circ} \mathrm{C} ; \mathrm{Cl}$, chloride; $\mathrm{NO}_{3}$, nitrate; $\mathrm{mg} / \mathrm{L}$, milligrams per liter; --, not measured; <, less than]

\begin{tabular}{|c|c|c|c|c|c|c|c|}
\hline $\begin{array}{l}\text { Well } \\
\text { no. }\end{array}$ & Owner & $\begin{array}{c}\text { Temperature } \\
\text { ( } \mathrm{C} \text { C) }\end{array}$ & $\begin{array}{c}\text { Specific } \\
\text { conductance } \\
(\mu \mathrm{S} / \mathrm{cm})\end{array}$ & pH & $\begin{array}{c}\mathrm{Cl} \\
(\mathrm{mg} / \mathrm{L})\end{array}$ & $\begin{array}{l}\mathrm{NO}_{3}, \\
\text { as N } \\
\text { (mg/L) }\end{array}$ & Remarks \\
\hline 1 & High School (1) & 27.5 & 1,700 & - & 320 & 11 & Major ion and trace element sample \\
\hline 2 & Ermes Smith & 28 & 1,280 & -- & - & 8 & \\
\hline 3 & High School (2) & 26.5 & 1,600 & -- & -- & 15 & \\
\hline 4 & High School (3) & 28 & 1,300 & - & - & 4 & \\
\hline 5 & High School (4) & 28 & 1,000 & -- & -- & 3 & \\
\hline 6 & Kulas (1) & 27.5 & 700 & - & -- & $<1$ & \\
\hline 7 & Kulas (2) & 27.5 & 680 & - & -- & 2 & \\
\hline 8 & Menas & 27.5 & 800 & - & -- & 7 & \\
\hline 9 & Felix & 27 & 800 & - & - & $<1$ & \\
\hline 10 & Fotomai & 27 & 875 & - & -- & $<1$ & \\
\hline 11 & Alphanso & 27.5 & 1,050 & - & -- & 2 & \\
\hline 12 & Magar & 28 & 1,050 & -- & -- & 4 & \\
\hline 13 & Keledo & 27.5 & 1,030 & - & - & 4 & \\
\hline 14 & Passano & 27.5 & 1,150 & - & - & $<1$ & \\
\hline 15 & Kipier (1) & 28 & 1,550 & -- & -- & 2 & \\
\hline 16 & Kipier (2) & 27.5 & 900 & - & -- & $<1$ & \\
\hline 17 & Wan & 27.5 & 1,750 & - & - & $<1$ & \\
\hline 18 & Ersin & 27.5 & 1,450 & -- & -- & 3 & \\
\hline 19 & Mateus & 26.5 & 1,600 & -- & - & 1 & \\
\hline 20 & Cox & 27.5 & 1,300 & - & 100 & 10 & \\
\hline 21 & Philip & 27 & - & -- & - & $<1$ & \\
\hline 22 & Konny & 27.5 & 825 & -- & -- & $<1$ & \\
\hline 23 & Anita & 27.5 & 1,250 & -- & -- & 3 & \\
\hline 24 & Thomas & 28 & 1,000 & -- & -- & 2 & \\
\hline 25 & Namichy & 27 & 1,000 & -- & -. & 1 & \\
\hline 26 & Changy & 27.5 & 1,600 & - & -- & 7 & \\
\hline 27 & Caspar & 27.5 & 1,000 & -- & - & 1 & \\
\hline 28 & Jacky (1) & 28 & 650 & - & -- & $<1$ & \\
\hline 29 & Jacky (2) & 27.5 & 975 & -- & - & 1 & \\
\hline 30 & Choring Rafial & 28.5 & 700 & -- & - & $<1$ & \\
\hline 31 & Ogi Rafial & 27.5 & 950 & - & - & $<1$ & \\
\hline 32 & Simeon & 28 & 760 & - & -- & $<1$ & \\
\hline 33 & Litong & 27.5 & 335 & -- & 21 & $<1$ & \\
\hline 34 & Sixto & 26.5 & 1,010 & -- & -- & $<1$ & \\
\hline 35 & Robert & 28 & 1,050 & -- & -- & $<1$ & \\
\hline 36 & Tanaka & 27.5 & 950 & -- & - & $<1$ & \\
\hline 37 & Solomon & 27 & 1,020 & - & - & $<1$ & \\
\hline 38 & Leon (1) & 28 & 1,080 & - & -- & $<1$ & \\
\hline 39 & Leon (2) & 28 & 780 & -- & -- & $<1$ & \\
\hline 40 & Rubano (1) & 28 & 1,280 & - & -- & 2 & \\
\hline 41 & Rubano (2) & 27 & 1,100 & -- & - & 1 & \\
\hline \multicolumn{2}{|c|}{ Taro patch } & 28 & 800 & - & - & -- & \\
\hline
\end{tabular}



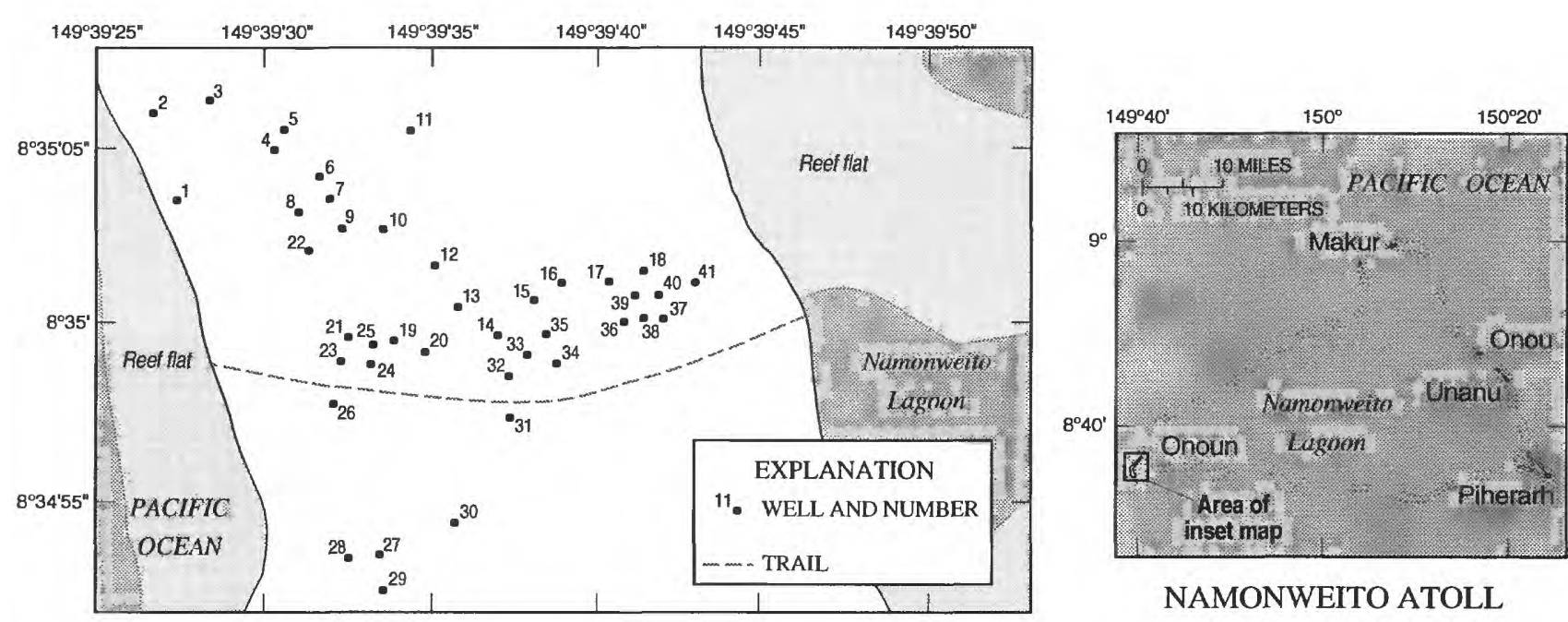

NAMONWEITO ATOLL
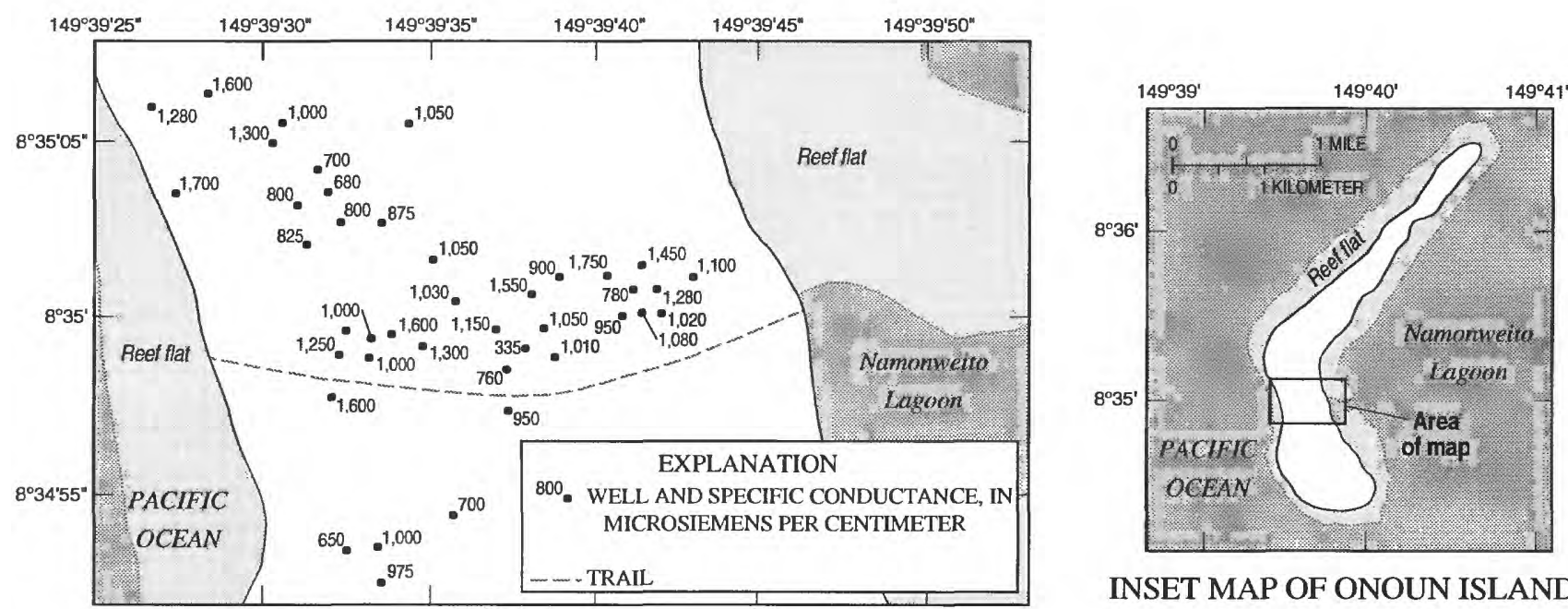

INSET MAP OF ONOUN ISLAND

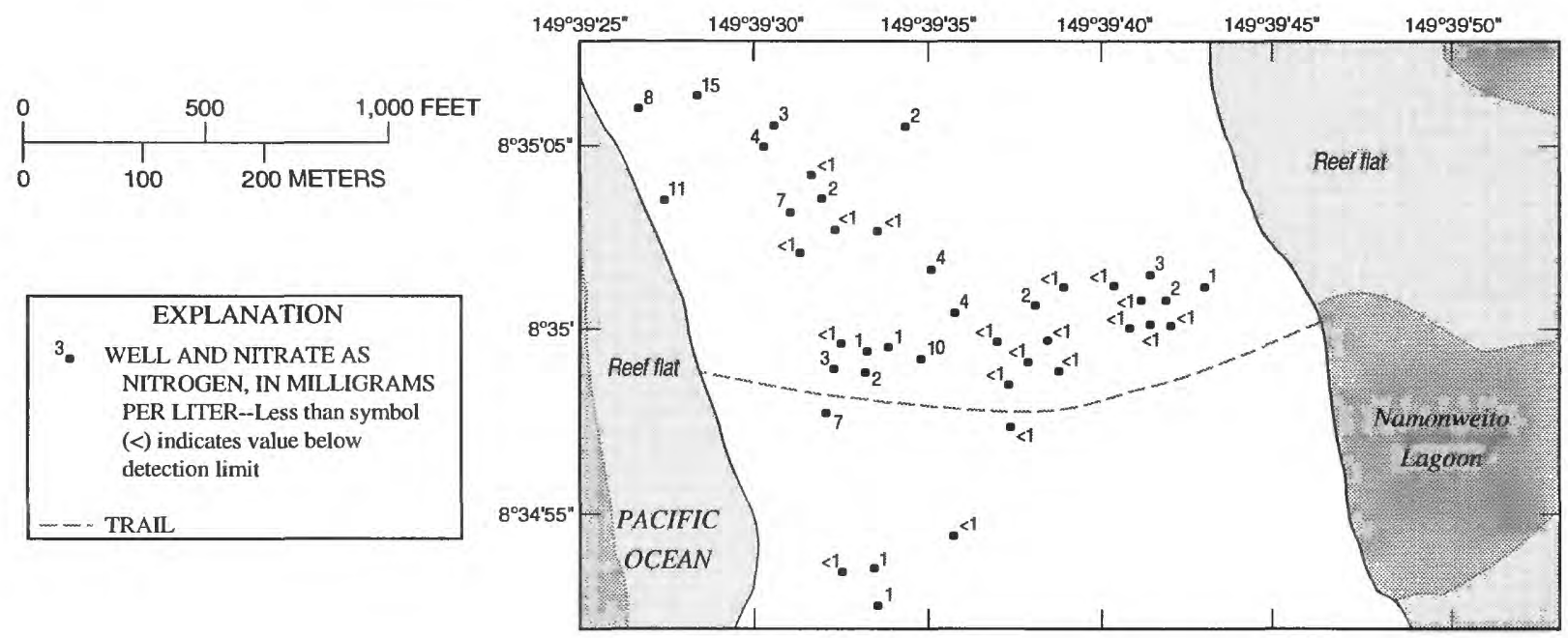

Figure 15. Well locations, specific conductance, and nitrate concentrations for Onoun Island, Namonweito Atoll, Namonweito Island Group, Chuuk State, 1984. 


\section{Makur Island}

The population of Makur Island, Namonweito Atoll is about 97 (Truk State Office of Planning and Statistics, 1981). Well locations, specific conductance, and nitrate concentrations for Makur Island are shown in figure 16. Selected physical and water-quality data for the 6 dug wells sampled are given in table 8 . The mean specific conductance for water from 6 wells was $1,220 \mu \mathrm{S} / \mathrm{cm}$ and ranged from 710 to $3,000 \mu \mathrm{S} / \mathrm{cm}$. On the basis of the estimated chloride concentration from specific conductance data, water from 1 of the 6 wells sampled (well number 6) exceeded the WHO (1984) guideline for chloride. All 6 of the wells sampled were field tested for nitrate concentration and yielded values ranging from 2 to $6 \mathrm{mg} / \mathrm{L}$ (as $\mathrm{N}$ ).

Table 8. Selected physical and water-quality data from dug wells, March 18, 1984, Makur Island, Namonweito Atoll, Namonweito Island Group

$\left[{ }^{\circ} \mathrm{C}\right.$, degrees Celsius; $\mu \mathrm{S} / \mathrm{cm}$, microsiemens per centimeter at $25^{\circ} \mathrm{C} ; \mathrm{Cl}$, chloride; $\mathrm{NO}_{3}$, nitrate; $\mathrm{mg} / \mathrm{L}$, milligrams per liter; --, not measured]

\begin{tabular}{|c|c|c|c|c|c|c|}
\hline Well no. & Owner & $\begin{array}{c}\text { Temperature } \\
\left({ }^{\circ} \mathrm{C}\right)\end{array}$ & $\begin{array}{c}\text { Specific } \\
\text { conductance } \\
(\mu S / \mathrm{cm})\end{array}$ & pH & $\begin{array}{c}\mathrm{Cl} \\
(\mathrm{mg} / \mathrm{L})\end{array}$ & $\begin{array}{c}\mathrm{NO}_{3}, \\
\text { as N } \\
(\mathrm{mg} / \mathrm{L})\end{array}$ \\
\hline 1 & Anton & 27.5 & 800 & -- & - & 6 \\
\hline 2 & Yasuo Smith & 28.5 & 710 & -- & - & 2 \\
\hline 3 & Public well & 27.5 & 1,050 & - & - & 3 \\
\hline 4 & Ananias & 28 & 750 & -- & - & 5 \\
\hline 5 & Teresa & 28.5 & 1,040 & - & - & 2 \\
\hline 6 & Osey Tom & 27 & 3,000 & -- & -- & 3 \\
\hline
\end{tabular}




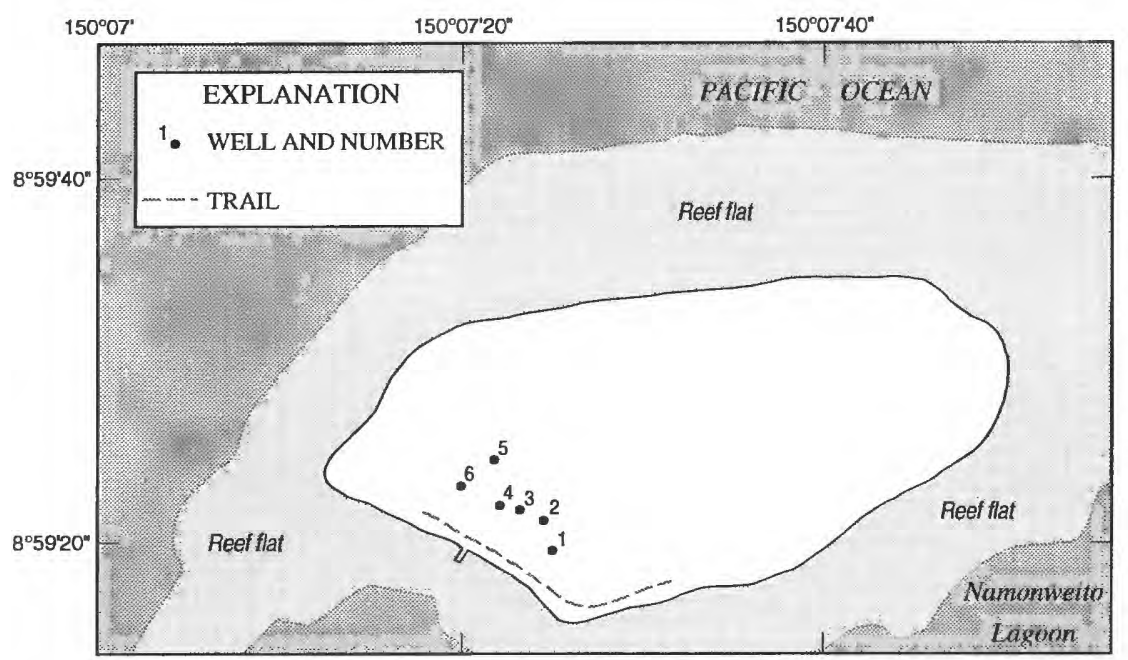

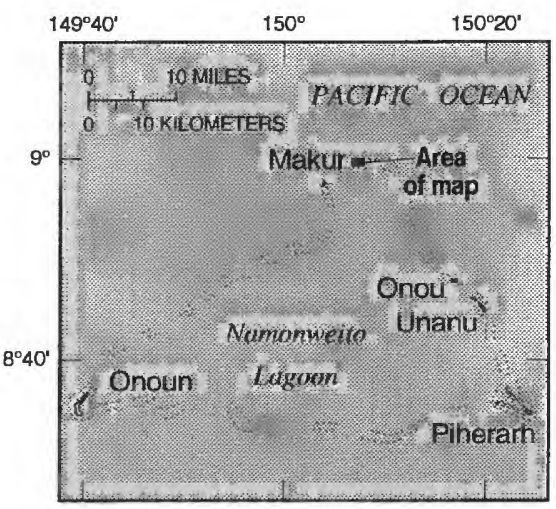

NAMONWEITO ATOLL
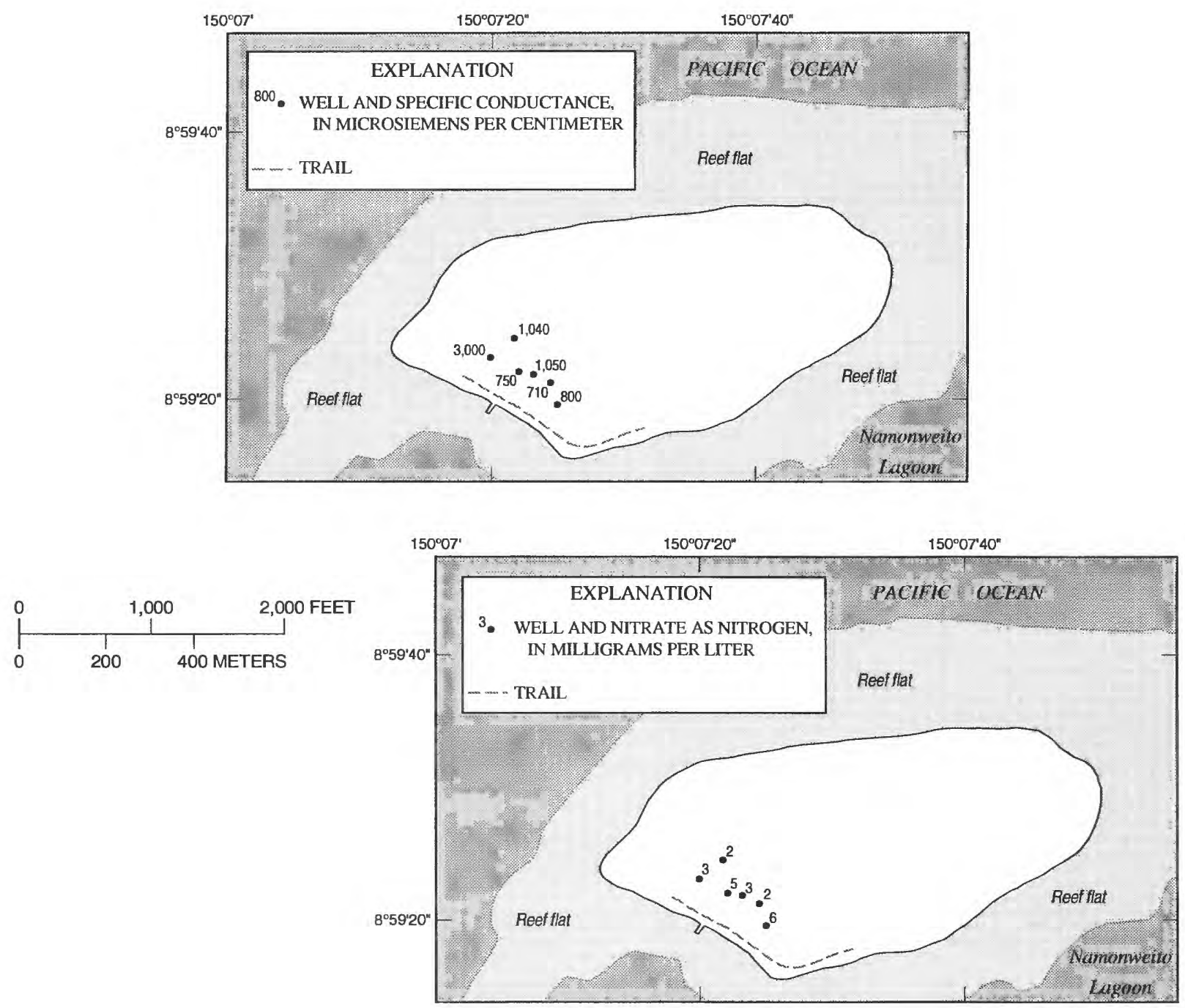

Figure 16. Well locations, specific conductance, and nitrate concentrations for Makur Island, Namonweito Atoll, Namonweito Island Group, Chuuk State, 1984. 


\section{Onou Island}

The population of Onou Island, Namonweito Atoll is about 59 (Truk State Office of Planning and Statistics, 1981). Well locations, sampling sites, specific conductance, and nitrate concentrations for Onou Island are shown in figure 17 . Selected physical and water-quality data for the 2 dug wells and 2 open pit sites sampled are given in table 9. The mean specific conductance for water from 2 wells was $6,750 \mu \mathrm{S} / \mathrm{cm}$ and ranged from 6,000 to $7,500 \mu \mathrm{S} / \mathrm{cm}$. On the basis of the estimated chloride concentrations from specific conductance data, water from both wells sampled exceeded the WHO (1984) guideline for chloride. Both of the wells sampled were field tested for nitrate concentration and yielded values of 1 and $2 \mathrm{mg} / \mathrm{L}$ (as $\mathrm{N}$ ).

Table 9. Selected physical and water-quality data from dug wells and miscellaneous sampling sites, March 19, 1984, Onou Island, Namonweito Atoll Group

$\left[{ }^{\circ} \mathrm{C}\right.$, degrees Celsius; $\mu \mathrm{S} / \mathrm{cm}$, microsiemens per centimeter at $25^{\circ} \mathrm{C} ; \mathrm{Cl}$, chloride; $\mathrm{NO}_{3}$, nitrate; $\mathrm{mg} / \mathrm{L}$, milligrams per liter; --, not measured]

\begin{tabular}{|c|c|c|c|c|c|c|}
\hline $\begin{array}{c}\text { Well no. } \\
\text { or sampling site }\end{array}$ & Owner & $\begin{array}{l}\text { Temperature } \\
\left.\text { ( }{ }^{\circ} \mathrm{C}\right)\end{array}$ & $\begin{array}{c}\text { Specific } \\
\text { conductance } \\
(\mu \mathrm{S} / \mathrm{cm})\end{array}$ & pH & $\begin{array}{c}\mathrm{Cl} \\
(\mathrm{mg} / \mathrm{L})\end{array}$ & $\begin{array}{c}\mathrm{NO}_{3} \\
\text { as N } \\
\text { (mg/L) }\end{array}$ \\
\hline 1 & Public well & 27.5 & 7,500 & -- & 2,000 & 2 \\
\hline 2 & Public well & 27.5 & 6,000 & -- & -- & 1 \\
\hline Open pit 1 & & 29 & 4,000 & -- & -- & -- \\
\hline Open pit 2 & & 27.5 & 6,000 & -- & -- & -- \\
\hline
\end{tabular}



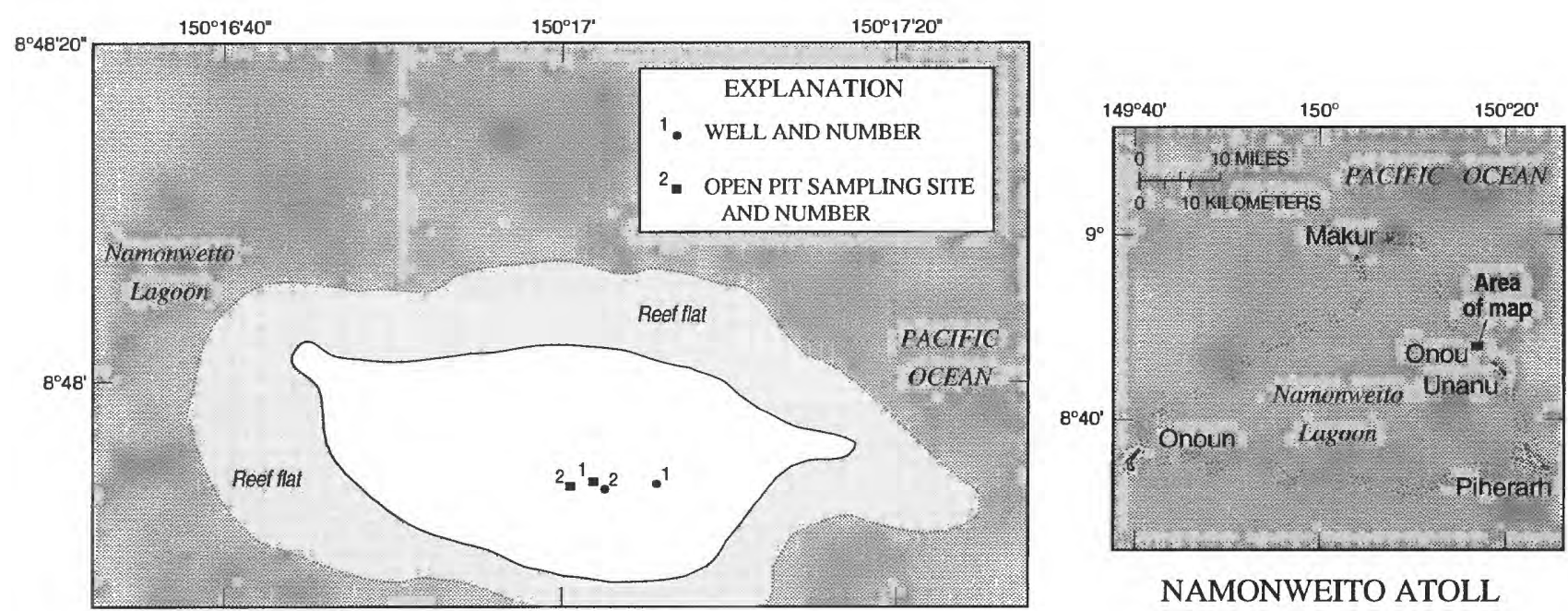

NAMONWEITO ATOLL
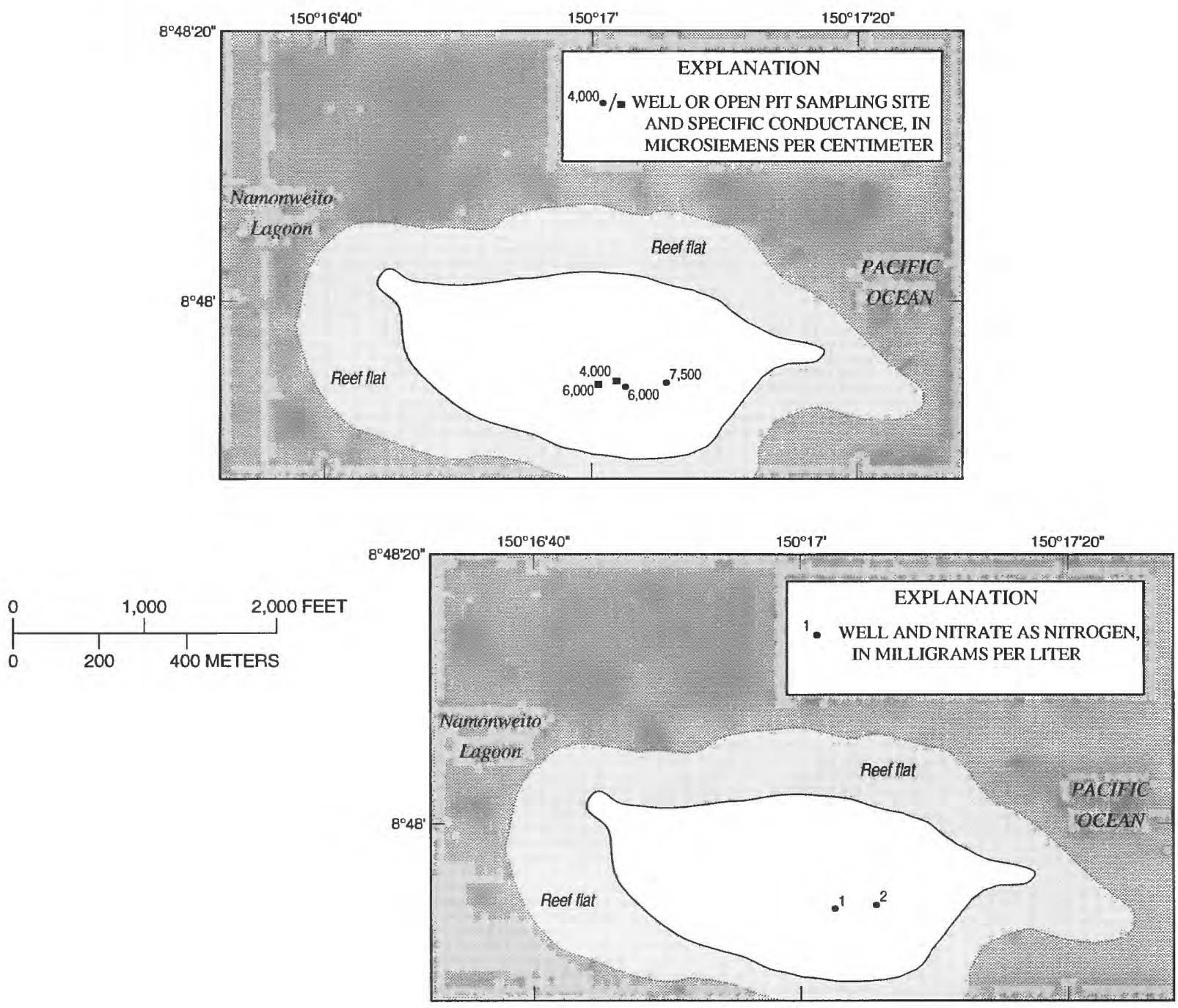

Figure 17. Well locations, specific conductance, and nitrate concentrations for Onou Island, Namonweito Atoll, Namonweito Island Group, Chuuk State, 1984. 


\section{Unanu Island}

The population of Unanu Island, Namonweito Atoll is about 79 (Truk State Office of Planning and Statistics, 1981). Well locations, specific conductance, and nitrate concentrations for Unanu Island are shown in figure 18. Selected physical and water-quality data for the 7 dug wells and 1 driven well sampled are given in table 10. The mean specific conductance for water from
8 wells was $3,340 \mu \mathrm{S} / \mathrm{cm}$ and ranged from 2,000 to $6,000 \mu \mathrm{S} / \mathrm{cm}$. On the basis of the estimated chloride concentrations from specific conductance data, all of these samples exceed the WHO (1984) guideline for chloride. Six of the 8 wells sampled were field tested for nitrate concentration. Water from 2 of the wells tested had nitrate concentrations of less than $1 \mathrm{mg} / \mathrm{L}$ (as $\mathrm{N}$ ). Nitrate concentrations for water from the other 4 wells tested ranged from 1 to $5 \mathrm{mg} / \mathrm{L}$ (as $\mathrm{N}$ ).

Table 10. Selected physical and water-quality data from dug and driven wells, March 20, 1984, Unanu Island, Namonweito Atoll, Namonweito Island Group

$\left[{ }^{\circ} \mathrm{C}\right.$, degrees Celsius; $\mu \mathrm{S} / \mathrm{cm}$, microsiemens per centimeter at $25^{\circ} \mathrm{C}$; $\mathrm{Cl}$, chloride; $\mathrm{NO}_{3}$, nitrate; $\mathrm{mg} / \mathrm{L}$, milligrams per liter, -, not measured; <, less than]

\begin{tabular}{|c|c|c|c|c|c|c|c|}
\hline $\begin{array}{l}\text { Well } \\
\text { no. }\end{array}$ & Owner & $\begin{array}{c}\text { Temperature } \\
\text { ('C) }\end{array}$ & $\begin{array}{c}\text { Specific } \\
\text { conductance } \\
(\mu \mathrm{S} / \mathrm{cm})\end{array}$ & pH & $\begin{array}{c}\mathrm{Cl} \\
(\mathrm{mg} / \mathrm{L})\end{array}$ & $\begin{array}{c}\mathrm{NO}_{3}, \\
\text { as N } \\
(\mathrm{mg} / \mathrm{L})\end{array}$ & Remarks \\
\hline 1 & Tara & 28 & 6,000 & - & - & 1 & \\
\hline 2 & Wan & 27.5 & 3,750 & -- & - & $<1$ & \\
\hline 3 & Osei & 28 & 3,500 & - & -- & - & \\
\hline 4 & Lucas & 27 & 3,000 & - & - & 5 & \\
\hline 5 & Public well & 27.5 & 2,000 & -- & - & 1 & \\
\hline 6 & Raimore & 27 & 2,100 & -- & - & - & \\
\hline 7 & Ananias & 27 & 2,900 & - & - & 4 & \\
\hline 8 & Chief & 26 & 3,500 & -- & - & $<1$ & Installed $11 / 4$ " well point with hand pump. \\
\hline
\end{tabular}



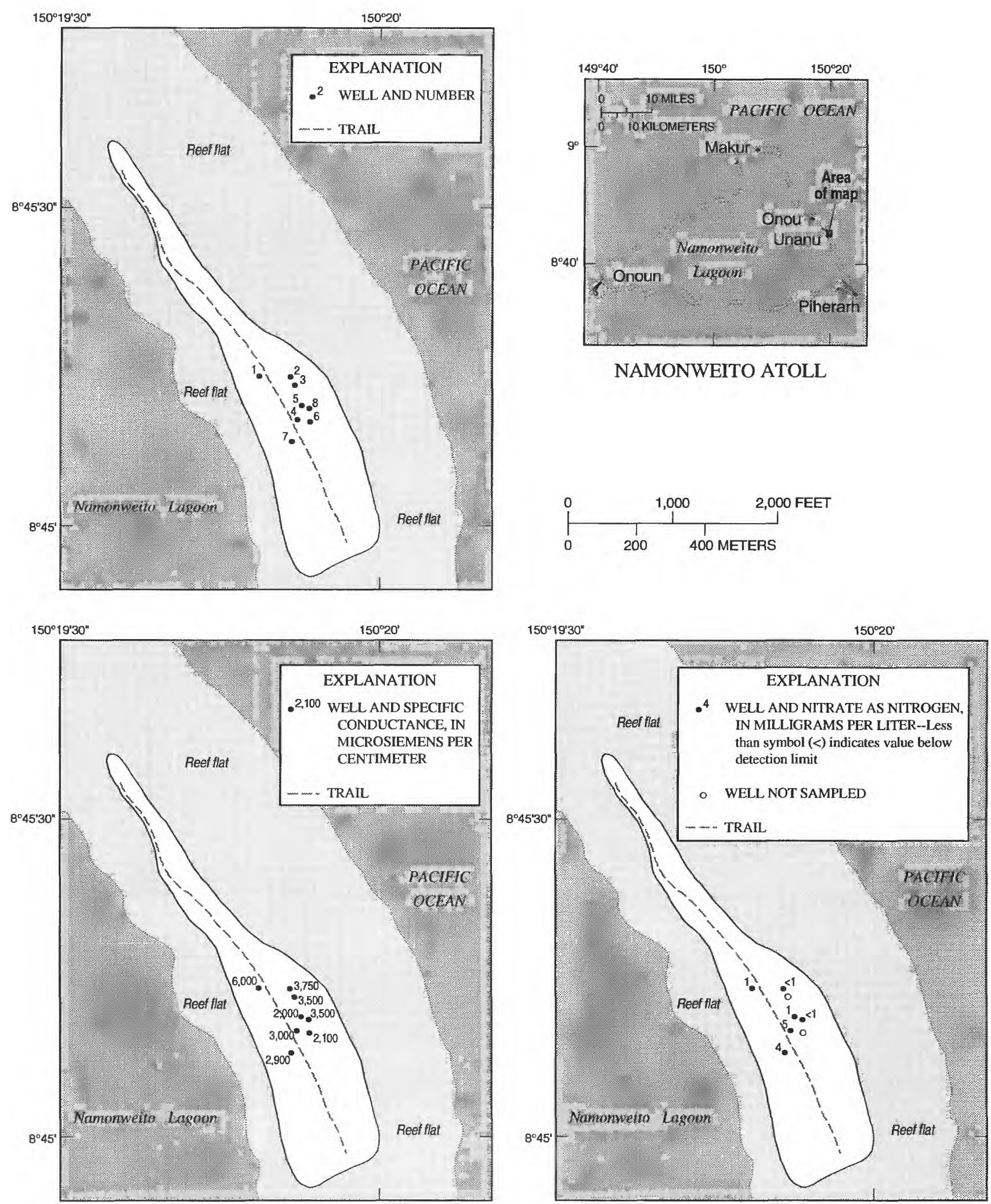

Figure 18. Well locations, specific conductance, and nitrate concentrations for Unanu Island, Namonweito Atoll, Namonweito Island Group, Chuuk State, 1984. 


\section{Piherarh Island}

The population of Piherarh Island, Namonweito Atoll is about 118 (Truk State Office of Planning and Statistics, 1981). Well locations, sampling sites, specific conductance, and nitrate concentrations for Piherarh Island are shown in figure 19. Selected physical and water-quality data for the 17 dug wells and 1 taro patch site sampled are given in table 11. The mean specific conductance for water from 17 wells was $1,790 \mu \mathrm{S} / \mathrm{cm}$ and ranged from 775 to $6,000 \mu \mathrm{S} / \mathrm{cm}$. On the basis of the estimated chloride concentrations from specific conductance data, water from 5 of the 17 wells (wells 2, 3, 12,16 , and 17) exceeded the WHO (1984) guideline for chloride. All 17 of the wells sampled were field tested for nitrate concentration, and yielded values ranging from 1 to $11 \mathrm{mg} / \mathrm{L}$ (as $\mathrm{N}$ ). Water from well 5 exceeded the WHO (1984) guideline for nitrate.

Table 11. Selected physical and water-quality data from dug wells and taro patch site, March 21, 1984, Piherarh Island, Namonweito Atoll, Namonweito Island Group

$\left[{ }^{\circ} \mathrm{C}\right.$, degrees Celsius; $\mu \mathrm{S} / \mathrm{cm}$, microsiemens per centimeter at $25^{\circ} \mathrm{C} ; \mathrm{Cl}$, chloride; $\mathrm{NO}_{3}$, nitrate; $\mathrm{mg} / \mathrm{L}$, milligrams per liter, --, not measured]

\begin{tabular}{|c|c|c|c|c|c|c|c|}
\hline $\begin{array}{c}\text { Well no. } \\
\text { or sampling } \\
\text { site }\end{array}$ & Owner & $\begin{array}{c}\text { Temperature } \\
\left({ }^{\circ} \mathrm{C}\right)\end{array}$ & $\begin{array}{c}\text { Specific } \\
\text { conductance } \\
(\mu \mathrm{S} / \mathrm{cm})\end{array}$ & pH & $\begin{array}{c}\mathrm{Cl} \\
\text { (mg/L) }\end{array}$ & $\begin{array}{c}\mathrm{NO}_{3}, \\
\text { as } \mathrm{N} \\
(\mathrm{mg} / \mathrm{L})\end{array}$ & Remarks \\
\hline 1 & Bius & 27.5 & 1,100 & - & - & 2 & \\
\hline 2 & Samuel & 27 & 1,600 & - & - & 2 & \\
\hline 3 & Maximino (1) & 27 & 6,000 & - & - & 4 & \\
\hline 4 & Maximino (2) & 27.5 & 800 & -- & - & 1 & \\
\hline 5 & Richy & 27.5 & 950 & 6.8 & 100 & 11 & Major ion and trace element sample \\
\hline 6 & Sikan & 27 & 1,020 & - & - & 1 & \\
\hline 7 & Nori & 27.5 & 1,300 & -- & - & 3 & \\
\hline 8 & Puruno & 27.5 & 775 & - & - & 3 & \\
\hline 9 & Poki & 27.5 & 1,100 & - & -- & 2 & \\
\hline 10 & Mateus & 27 & 925 & - & - & 5 & \\
\hline 11 & Luciano & 27.5 & 825 & -- & -- & 2 & \\
\hline 12 & Eramus & 27 & 1,600 & - & -- & 8 & \\
\hline 13 & Yowanis & 27 & 960 & -- & -- & 5 & \\
\hline 14 & Emere & 27 & 960 & - & - & 2 & \\
\hline 15 & Teruo (1) & 27 & 785 & - & -- & 4 & \\
\hline 16 & Teruo (2) & 28 & 3,700 & - & - & 3 & \\
\hline 17 & Pisente & 27.5 & 6,000 & - & - & 1 & \\
\hline Taro patch & & 28 & 850 & - & - & 1 & \\
\hline
\end{tabular}



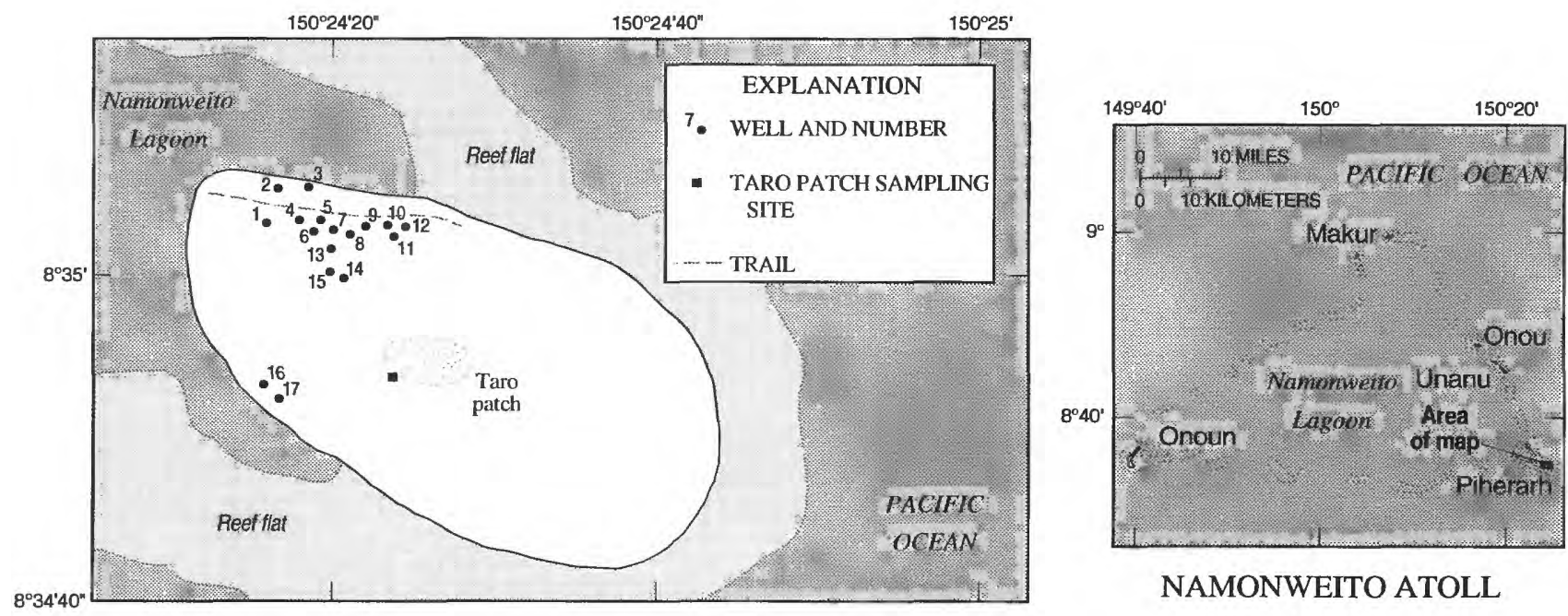

NAMONWEITO ATOLL
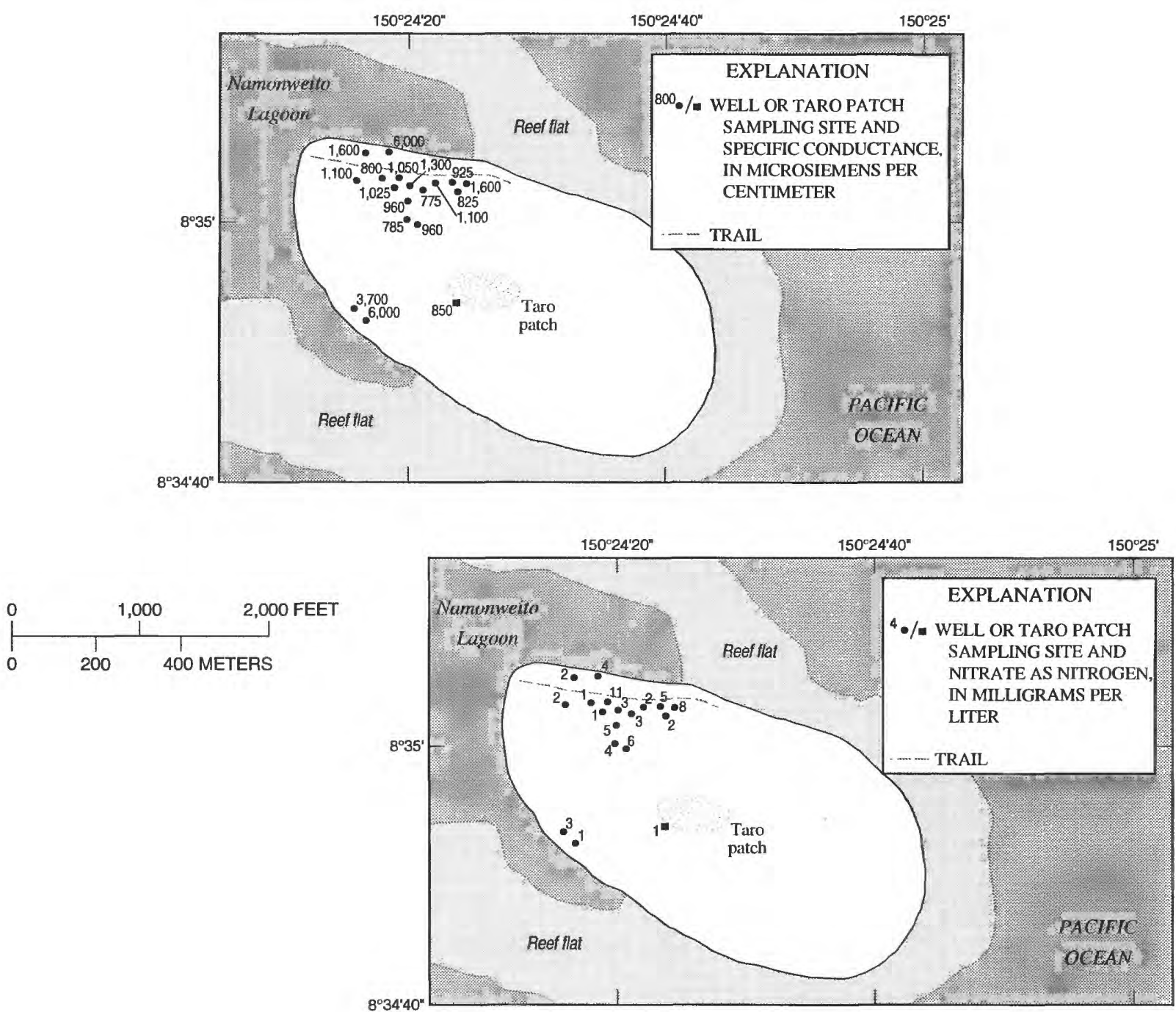

Figure 19. Well locations, specific conductance, and nitrate concentrations for Piherarh Island, Namonweito Atoll, Namonweito Island Group, Chuuk State, 1984. 


\section{Hall Island Group}

The inhabited islands of the Hall Island Group are Nomwin and Fananu Islands at Nomwin Atoll, and Murilo and Ruo Islands at Murilo Atoll (fig. 20). These islands are about $80 \mathrm{mi}$ north of the Chuuk Island Group. Mean values for specific conductance of ground water on each of the islands range from $1,120 \mu \mathrm{S} / \mathrm{cm}$ on
Murilo Island to $2,060 \mu \mathrm{S} / \mathrm{cm}$ on Fananu Island. Field determinations for nitrate concentration of ground water ranged from less than $1 \mathrm{mg} / \mathrm{L}$ (as $\mathrm{N}$ ) at selected sites on all islands to $18 \mathrm{mg} / \mathrm{L}$ (as N) on Nomwin Island. Table 12 includes physical, major-ion, and trace-element data for selected wells in the Hall Island Group. Specific information for each of the islands is presented below.

Table 12. Physical, major ion, and trace element data for selected wells in the Hall Island Group, Chuuk State $\left[{ }^{\circ} \mathrm{C}\right.$, degrees Celsius; $\mu \mathrm{S} / \mathrm{cm}$, microsiemens per centimeter at $25^{\circ} \mathrm{C} ; \mathrm{mg} / \mathrm{L}$, milligrams per liter; $\mu \mathrm{g} / \mathrm{L}$, micrograms per liter, $\mathrm{CaCO}_{3}, \mathrm{Calcium}$-carbonate; --, not measured; $<$, less than]

\begin{tabular}{|c|c|c|c|c|c|}
\hline Property or constituent & Units & $\begin{array}{c}\text { Nomwin } \\
\text { well } 2 \\
03 / 16 / 84\end{array}$ & $\begin{array}{c}\text { Fananu } \\
\text { well } 6 \\
03 / 16 / 84\end{array}$ & $\begin{array}{c}\text { Murilo } \\
\text { well 14 } \\
03 / 04 / 84\end{array}$ & $\begin{array}{c}\text { Ruo } \\
\text { well } 4 \\
03 / 04 / 84\end{array}$ \\
\hline Specific conductance $\ldots \ldots \ldots \ldots$ & $\mu \mathrm{S} / \mathrm{cm}$ & 1,900 & 4,000 & 975 & 1,550 \\
\hline Temperature............... & ${ }^{\circ} \mathrm{C}$ & 28.0 & 27.5 & 27.0 & 27.0 \\
\hline Hardness as $\mathrm{CaCO}_{3}$ & $\mathrm{mg} / \mathrm{L}$ & 500 & 660 & 370 & 500 \\
\hline Sodium, total as $\mathrm{Na} . . . \ldots \ldots \ldots$ & $\mathrm{mg} / \mathrm{L}$ & 160 & 490 & 63 & 140 \\
\hline Potassium, total as $\mathrm{K} \ldots \ldots \ldots \ldots$ & $\mathrm{mg} / \mathrm{L}$ & 71 & 20 & 2.7 & 7.8 \\
\hline Alkalinity, total as $\mathrm{CaCO}_{3} \ldots$ & $\mathrm{mg} / \mathrm{L}$ & 485 & 366 & 358 & 484 \\
\hline Sulfate, dissolved as $\mathrm{SO}_{4}$ & $\mathrm{mg} / \mathrm{L}$ & 63 & 150 & 20 & 71 \\
\hline Aluminum, total as $\mathrm{Al} \ldots \ldots \ldots \ldots$ & $\mathrm{mg} / \mathrm{L}$ & 40 & 30 & 30 & 20 \\
\hline Arsenic, total as As . . . . . . . . & $\mu \mathrm{g} / \mathrm{L}$ & 2 & 1 & $<1$ & 4 \\
\hline Barium, total as $\mathrm{Ba} \ldots \ldots \ldots \ldots$ & $\mu \mathrm{g} / \mathrm{L}$ & $<100$ & $<100$ & $<100$ & $<100$ \\
\hline Beryllium, total as $\mathrm{Be} . \ldots \ldots \ldots$. & $\mu \mathrm{g} / \mathrm{L}$ & $<10$ & $<10$ & $<10$ & $<10$ \\
\hline Cadmium, total as $\mathrm{Cd} \ldots \ldots \ldots \ldots$ & $\mu \mathrm{g} / \mathrm{L}$ & $<1$ & $<1$ & $<1$ & 3 \\
\hline Chromium, total as $\mathrm{Cr} \ldots \ldots \ldots \ldots$ & $\mu \mathrm{g} / \mathrm{L}$ & 20 & 20 & $<10$ & 10 \\
\hline Cobalt, total as Co............ & $\mu \mathrm{g} / \mathrm{L}$ & $<1$ & $<1$ & 1 & $<1$ \\
\hline Copper, total as $\mathrm{Cu} \ldots \ldots \ldots \ldots \ldots$ & $\mu \mathrm{g} / \mathrm{L}$ & 6 & 2 & 2 & 5 \\
\hline Mercury, total as $\mathrm{Hg} \ldots \ldots \ldots \ldots$ & $\mu \mathrm{g} / \mathrm{L}$ & 0.1 & 0.1 & $<0.1$ & $<0.1$ \\
\hline Molybdenum, total as Mo ........ & $\mathrm{m} / \mathrm{L}$ & 1 & $<1$ & $<1$ & $<1$ \\
\hline Nickel, total as N............ & $\mu \mathrm{g} / \mathrm{L}$ & 2 & $<1$ & 3 & 4 \\
\hline Selenium, total as $\mathrm{Se} . \ldots \ldots \ldots \ldots$ & $\mu \mathrm{g} / \mathrm{L}$ & $<1$ & $<1$ & $<1$ & $<1$ \\
\hline Silver, total as $\mathrm{Ag} \ldots \ldots \ldots \ldots$. & $\mu \mathrm{g} / \mathrm{L}$ & $<1$ & $<1$ & $<1$ & $<1$ \\
\hline Zinc, total as Zn............. & $\mu \mathrm{g} / \mathrm{L}$ & 20 & 30 & 20 & 40 \\
\hline
\end{tabular}




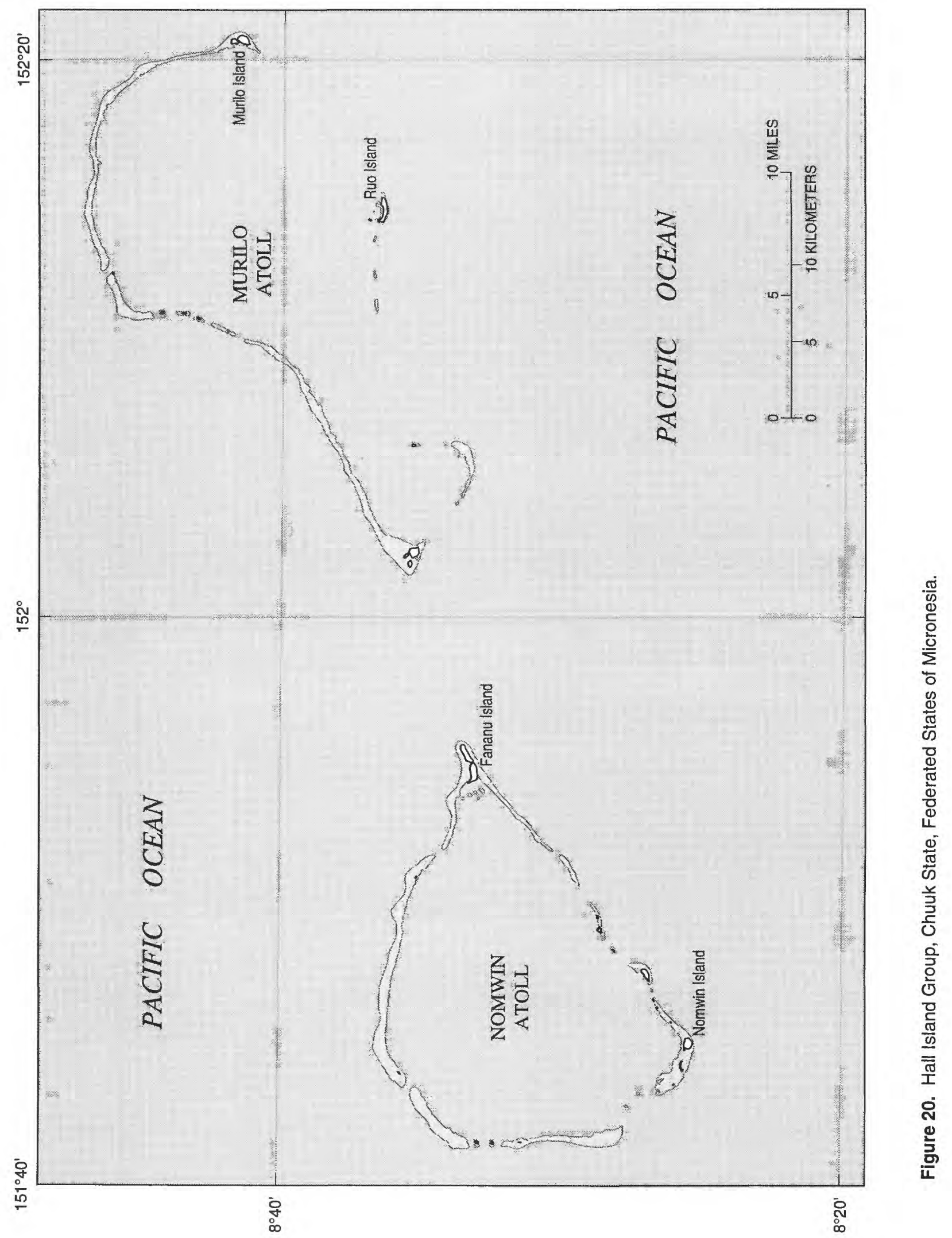




\section{Nomwin Island}

The population of Nomwin Island at Nomwin Atoll is about 324 (Truk State Office of Planning and Statistics, 1981). Well locations, specific conductance, and nitrate concentrations for Nomwin Island are shown in figure 21 . Selected physical and water-quality data for the 24 dug wells sampled are given in table 13 . The mean specific conductance for water from 24 wells was $1,210 \mu \mathrm{S} / \mathrm{cm}$ and ranged from 580 to $2,700 \mu \mathrm{S} / \mathrm{cm}$. On the basis of the estimated chloride concentrations from specific conductance data, water from 4 of the 24 wells sampled (wells 2, 9, 21, and 22) exceeded the WHO (1984) guideline for chloride. Seventeen of the 24 wells sampled were field tested for nitrate concentration. Water from about half the wells tested had nitrate concentrations of less than $1 \mathrm{mg} / \mathrm{L}$ (as $\mathrm{N}$ ); the others ranged from 2 to $18 \mathrm{mg} / \mathrm{L}$ (as $\mathrm{N}$ ). Two of these water samples (wells 2 and 9) exceeded the WHO (1984) guideline for nitrate.

Table 13. Selected physical and water-quality data from dug wells, March 16, 1984, Nomwin Island, Nomwin Atoll, Hall Island Group

$\left[{ }^{\circ} \mathrm{C}\right.$, degrees Celsius; $\mu \mathrm{S} / \mathrm{cm}$, microsiemens per centimeter at $25^{\circ} \mathrm{C} ; \mathrm{Cl}$, chloride; $\mathrm{NO}_{3}$, nitrate; $\mathrm{mg} / \mathrm{L}$, milligrams per liter; --, not measured; <, less than]

\begin{tabular}{|c|c|c|c|c|c|c|c|}
\hline $\begin{array}{l}\text { Well } \\
\text { no. }\end{array}$ & Owner & $\begin{array}{c}\text { Temperature } \\
\left({ }^{\circ} \mathrm{C}\right)\end{array}$ & $\begin{array}{c}\text { Specific } \\
\text { conductance } \\
(\mu \mathrm{S} / \mathrm{cm})\end{array}$ & pH & $\begin{array}{c}\mathrm{Cl} \\
\text { (mg/L) }\end{array}$ & $\begin{array}{c}\mathrm{NO}_{3}, \\
\text { as } \mathrm{N} \\
(\mathrm{mg} / \mathrm{L})\end{array}$ & Remarks \\
\hline 1 & Sokichy & 27.5 & 1,050 & - & - & $<1$ & \\
\hline 2 & Kotlip & 28 & 1,900 & 6.9 & 260 & 16 & Major ion and trace element sample \\
\hline 3 & Pungopung & 28 & 950 & - & - & $<1$ & \\
\hline 4 & Nisuo & 28 & 910 & - & - & -- & \\
\hline 5 & Faniap & 27 & 1,000 & -- & -- & $<1$ & \\
\hline 6 & Snyder & 28 & 1,200 & -- & -- & $<1$ & \\
\hline 7 & Robert & 27 & 780 & -- & -- & $<1$ & \\
\hline 8 & Sadao & 27 & 850 & - & - & $<1$ & \\
\hline 9 & James & 28 & 2,000 & -- & 240 & 18 & \\
\hline 10 & Ruber & 28 & 1,200 & - & -- & $<1$ & \\
\hline 11 & Lifang & 27 & 1,050 & -- & -- & 2 & \\
\hline 12 & Secy & 30 & 700 & - & -- & 3 & \\
\hline 13 & Kisano & 28.5 & 580 & - & - & 3 & \\
\hline 14 & Menchor & 27.5 & 800 & - & -- & -- & \\
\hline 15 & Helmut & 27 & 950 & -- & - & $<1$ & \\
\hline 16 & Koma & 27.5 & 1,120 & -- & -- & 5 & \\
\hline 17 & Alita & 27.5 & 1,320 & - & - & - & \\
\hline 18 & Pweta & 27 & 1,400 & -- & - & 4 & \\
\hline 19 & Fiman & 27.5 & 1,100 & - & -- & -- & \\
\hline 20 & Kimuo & 27 & 1,100 & -- & - & 2 & \\
\hline 21 & Sopian & 28 & 2,300 & - & 490 & - & \\
\hline 22 & Otto & 27 & 2,700 & -- & -- & -- & \\
\hline 23 & Malul & 27.5 & 1,350 & -- & -- & 2 & \\
\hline 24 & Yoichy & 29 & 700 & - & - & -- & \\
\hline
\end{tabular}



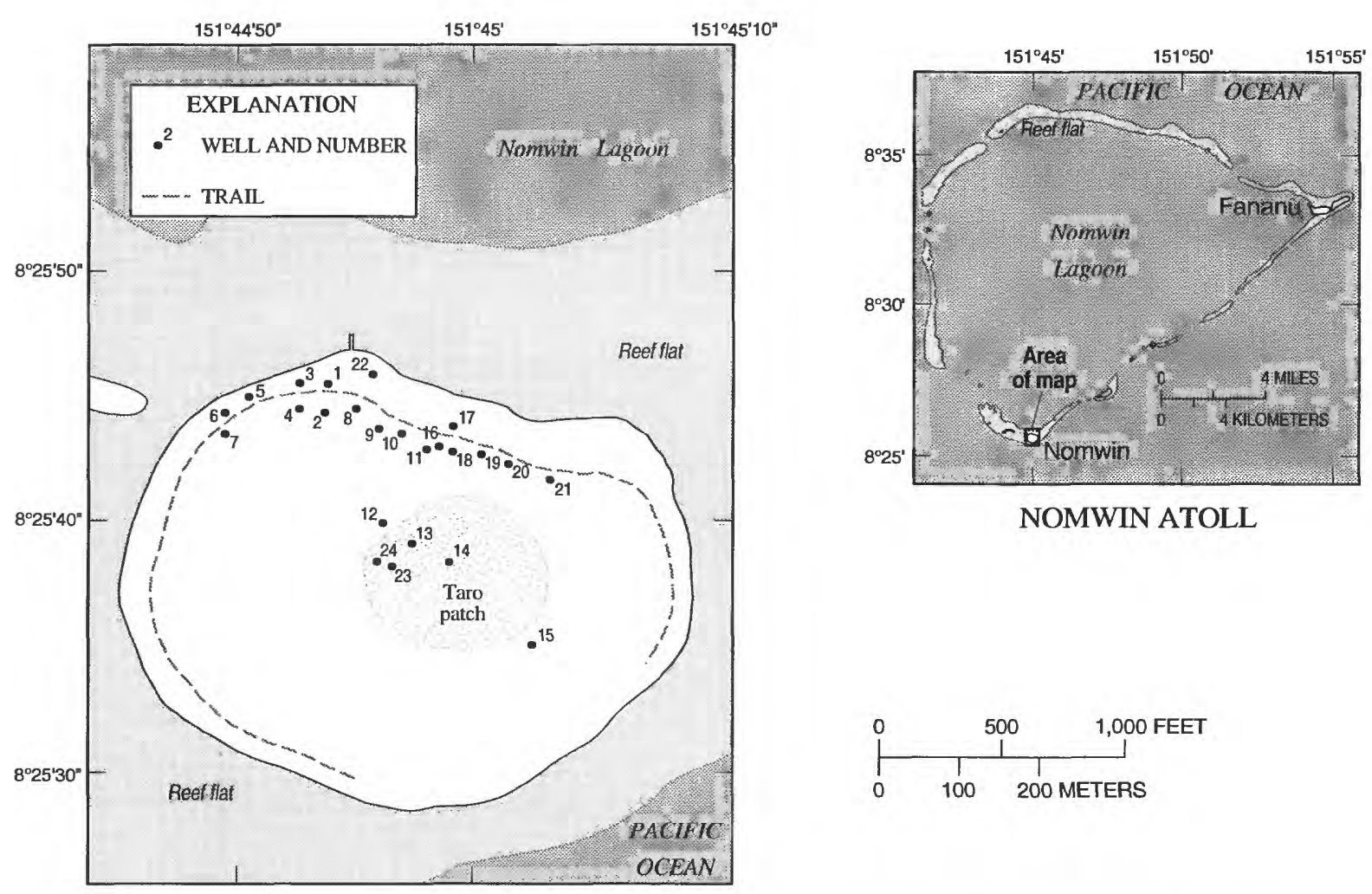

NOMWIN ATOLL
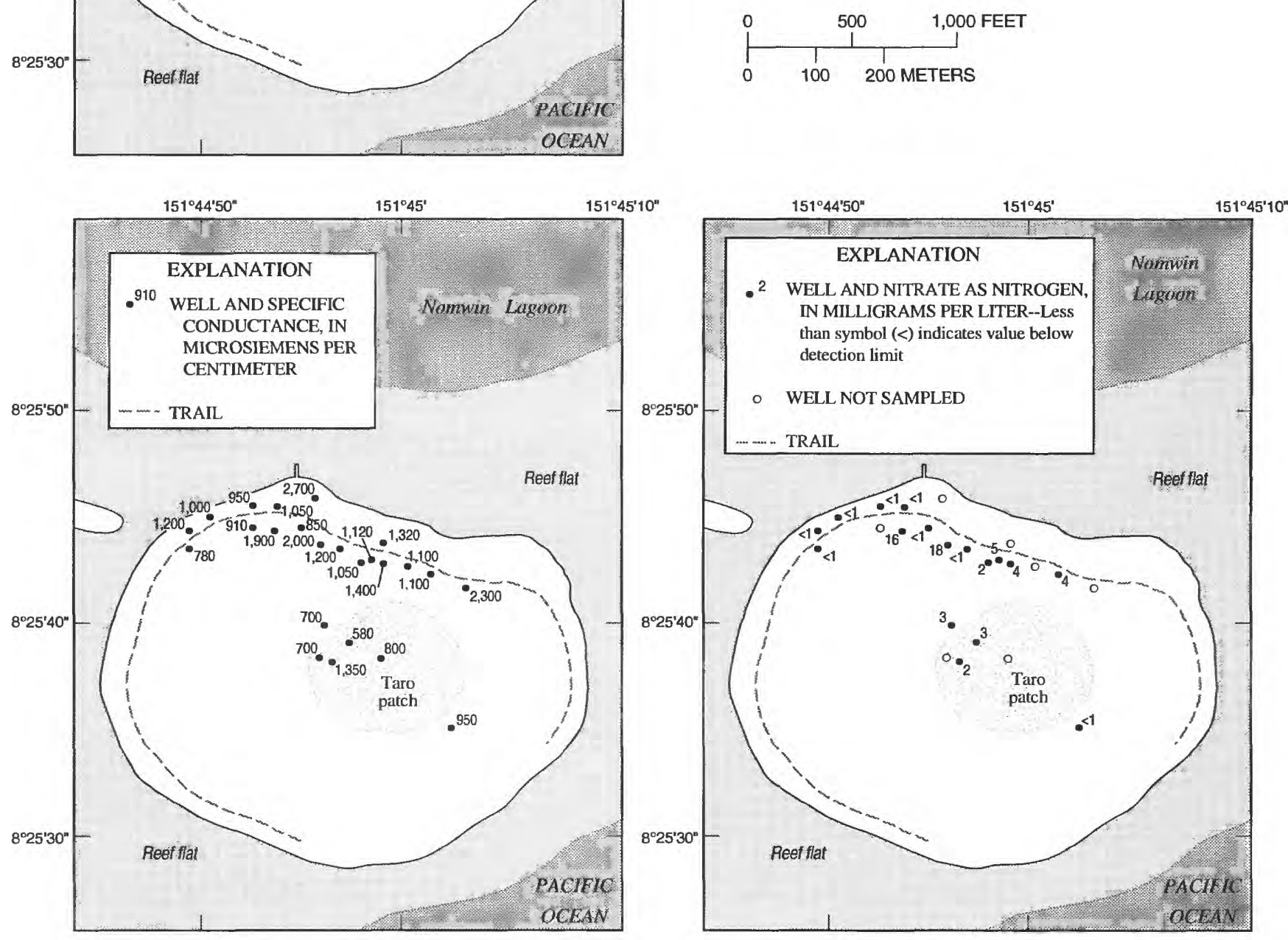

Figure 21. Well locations, specific conductance, and nitrate concentrations for Nomwin Island, Nomwin Atoll, Hall Island Group, Chuuk State, 1984. 


\section{Fananu Island}

The population of Fananu Island at Nomwin Atoll is about 238 (Truk State Office of Planning and Statistics, 1981). Well locations, sampling sites, specific conductance, and nitrate concentrations for Fananu Island are shown in figure 22 . Selected physical and waterquality data for the 10 dug wells and 4 taro patch sites sampled are given in table 14 . The mean specific conductance for water from 10 wells was $2,060 \mu \mathrm{S} / \mathrm{cm}$ and ranged from 600 to $4,000 \mu \mathrm{S} / \mathrm{cm}$. On the basis of the estimated chloride concentrations from specific conductance data, water from 8 of the 10 wells sampled (wells 1 through 8) exceeded the WHO (1984) guideline for chloride. The highest measured value of specific conductance $(>8,000 \mu \mathrm{S} / \mathrm{cm})$ was for water collected from a small taro patch near the north shore of the island. Nine of the 10 wells sampled were field tested for nitrate concentration, and about half the water samples had values less than $1 \mathrm{mg} / \mathrm{L}$ (as $\mathrm{N}$ ); the others ranged from 2 to $4 \mathrm{mg} / \mathrm{L}$ (as $\mathrm{N}$ ).

Table 14. Selected physical and water-quality data from dug wells and taro patch sites, March 16, 1984, Fananu Island, Nomwin Atoll, Hall Island Group

$\left[{ }^{\circ} \mathrm{C}\right.$, degrees Celsius; $\mu \mathrm{S} / \mathrm{cm}$, microsiemens per centimeter at $25^{\circ} \mathrm{C} ; \mathrm{Cl}$, chloride; $\mathrm{NO}_{3}$, nitrate; $\mathrm{mg} / \mathrm{L}$, milligrams per liter; --, not measured; <, less than; $>$, greater than]

\begin{tabular}{|c|c|c|c|c|c|c|c|}
\hline $\begin{array}{l}\text { Well no. } \\
\text { or sam- } \\
\text { pling site }\end{array}$ & Owner & $\begin{array}{c}\text { Temperature } \\
\left({ }^{\circ} \mathrm{C}\right)\end{array}$ & $\begin{array}{c}\text { Specific } \\
\text { conductance } \\
(\mu \mathrm{S} / \mathrm{cm})\end{array}$ & pH & $\begin{array}{c}\mathrm{Cl} \\
(\mathrm{mg} / \mathrm{L})\end{array}$ & $\begin{array}{c}\mathrm{NO}_{3}, \\
\text { as N } \\
\text { (mg/L) }\end{array}$ & Remarks \\
\hline 1 & Abel & 26.5 & 1,850 & - & - & 4 & \\
\hline 2 & Naruo & 26.5 & 2,000 & - & -- & $<1$ & \\
\hline 3 & Sipura & 26 & 2,200 & -- & - & $<1$ & \\
\hline 4 & Sona & 27 & 1,700 & - & -- & $<1$ & \\
\hline 5 & Sopi & 27.5 & 2,150 & - & -- & 3 & \\
\hline 6 & Akkin & 27.5 & 4,000 & 6.9 & 930 & 3 & Major ion and trace element sample \\
\hline 7 & Aky & 27 & 2,700 & - & -- & 2 & \\
\hline 8 & Aitel & 27 & 2,100 & - & - & $<1$ & \\
\hline 9 & Matou & 27 & 1,350 & - & -- & -. & \\
\hline 10 & Sobi & 27 & 600 & - & - & 2 & \\
\hline \multicolumn{2}{|c|}{ Taro patch 1} & 26.5 & 2,100 & - & - & -- & \\
\hline \multirow{4}{*}{\multicolumn{2}{|c|}{ Taro patch 2}} & 29 & 420 & - & - & - & \\
\hline & & & 540 & -- & - & - & \\
\hline & & & 390 & -. & -- & - & \\
\hline & & & 660 & - & -- & -- & \\
\hline \multicolumn{2}{|c|}{ Taro patch 3} & 27 & 740 & - & - & - & \\
\hline \multicolumn{2}{|c|}{ Taro patch 4} & 29.5 & $>8,000$ & - & - & -- & \\
\hline
\end{tabular}



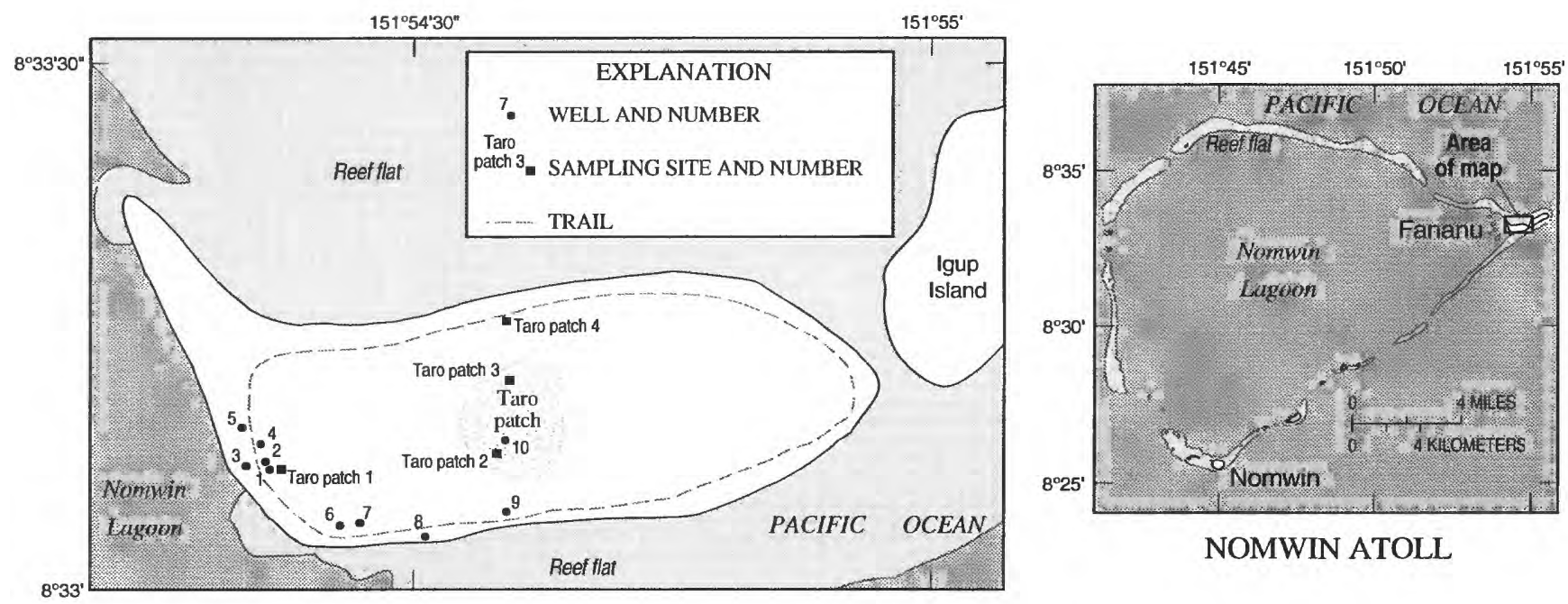

NOMWIN ATOLL
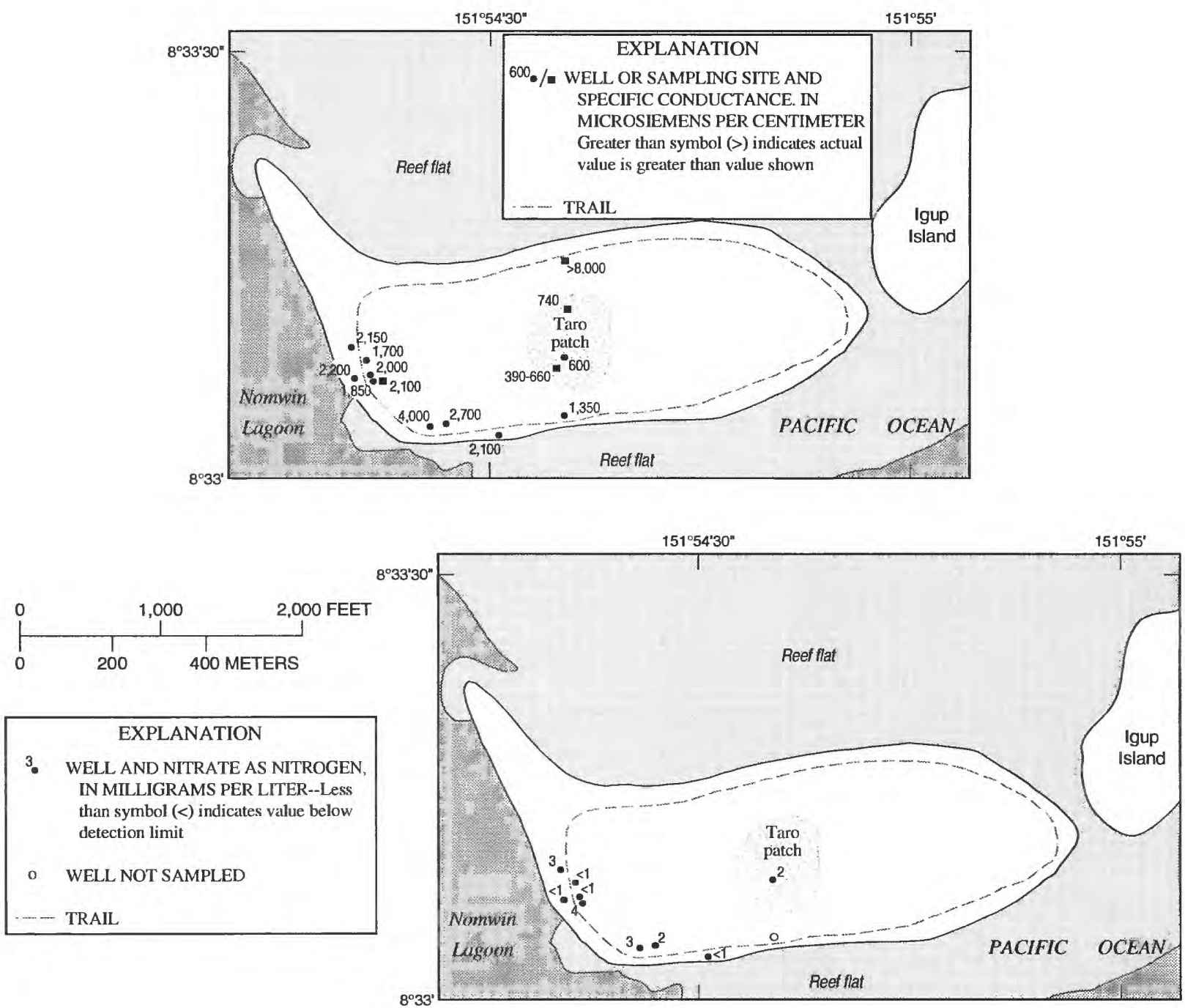

Figure 22. Well locations, specific conductance, and nitrate concentrations for Fananu Island, Nomwim Atoll, Hall Island Group, Chuuk State, 1984. 


\section{Murilo Island}

The population of Murilo Island at Murilo Atoll is about 330 (Truk State Office of Planning and Statistics, 1981). Well locations, specific conductance, and nitrate concentrations for Murilo Island are shown in figure 23. Selected physical and water-quality data for the $15 \mathrm{dug}$ wells sampled are given in table 15 . The mean specific conductance for water from 15 wells was $1,120 \mu \mathrm{S} / \mathrm{cm}$ and ranged from 650 to $1,950 \mu \mathrm{S} / \mathrm{cm}$. On the basis of the estimated chloride concentrations from specific conductance data, water from 2 of the 15 wells sampled (wells 2 and 7) exceeded the WHO (1984) guideline for chloride. Seven of the 10 wells sampled were field tested for nitrate concentration. Water from 3 of 7 the wells tested had nitrate concentrations of less than $1 \mathrm{mg} / \mathrm{L}$ (as $\mathrm{N}$ ); the others had concentrations ranging from 2 to 10 $\mathrm{mg} / \mathrm{L}$ (as N).

Table 15. Selected physical and water-quality data from dug wells, March 4, 1984, Murilo Island, Murilo Atoll, Hall Island Group $\left[{ }^{\circ} \mathrm{C}\right.$, degrees Celsius; $\mu \mathrm{S} / \mathrm{cm}$, microsiemens per centimeter at $25^{\circ} \mathrm{C} ; \mathrm{Cl}$, chloride; $\mathrm{NO}_{3}$, nitrate; $\mathrm{mg} / \mathrm{L}$, milligrams per liter, --, not measured; <, less than]

\begin{tabular}{|c|c|c|c|c|c|c|c|}
\hline $\begin{array}{l}\text { Well } \\
\text { no. }\end{array}$ & Owner & $\begin{array}{c}\text { Temperature } \\
\left({ }^{\circ} \mathrm{C}\right)\end{array}$ & $\begin{array}{c}\text { Specific } \\
\text { conductance } \\
(\mu \mathrm{S} / \mathrm{cm})\end{array}$ & pH & $\underset{(\mathrm{mg} / L)}{\mathrm{Cl}}$ & $\begin{array}{l}\mathrm{NO}_{3}, \\
\text { as N } \\
(\mathrm{mg} / \mathrm{L})\end{array}$ & Remarks \\
\hline 1 & Itet & 27.0 & 1,400 & 6.9 & 220 & -- & \\
\hline 2 & Micky & 26.5 & 1,750 & 6.9 & -- & -- & \\
\hline 3 & Rotcnis & 26.5 & 925 & 7.0 & -- & 3 & \\
\hline 4 & Kawa & 26.5 & 900 & 6.9 & 75 & -- & \\
\hline 5 & Benjamin & 26.5 & 1,100 & 6.9 & -- & -- & \\
\hline 6 & Marcos & 26.5 & 850 & 7.0 & -- & 2 & \\
\hline 7 & Jonas & 27 & 1,950 & -- & 340 & -- & \\
\hline 8 & Ellas & 27 & 1,400 & 6.6 & -- & 10 & \\
\hline 9 & Dennis & 27 & 875 & 6.8 & -- & -- & \\
\hline 10 & Thomas & 27 & 780 & 7.2 & -- & 3 & \\
\hline 11 & Amita & 27 & 650 & 7.2 & - & -- & \\
\hline 12 & Tereky & 27 & 1,050 & 6.9 & -- & $<1$ & \\
\hline 13 & Take & 26.5 & 1,400 & 7.3 & -- & -- & \\
\hline 14 & Near & 27 & 975 & 7.1 & -- & $<1$ & Major ion and trace element sample \\
\hline 15 & Aunu & 27 & 750 & 7.1 & -- & $<1$ & \\
\hline
\end{tabular}



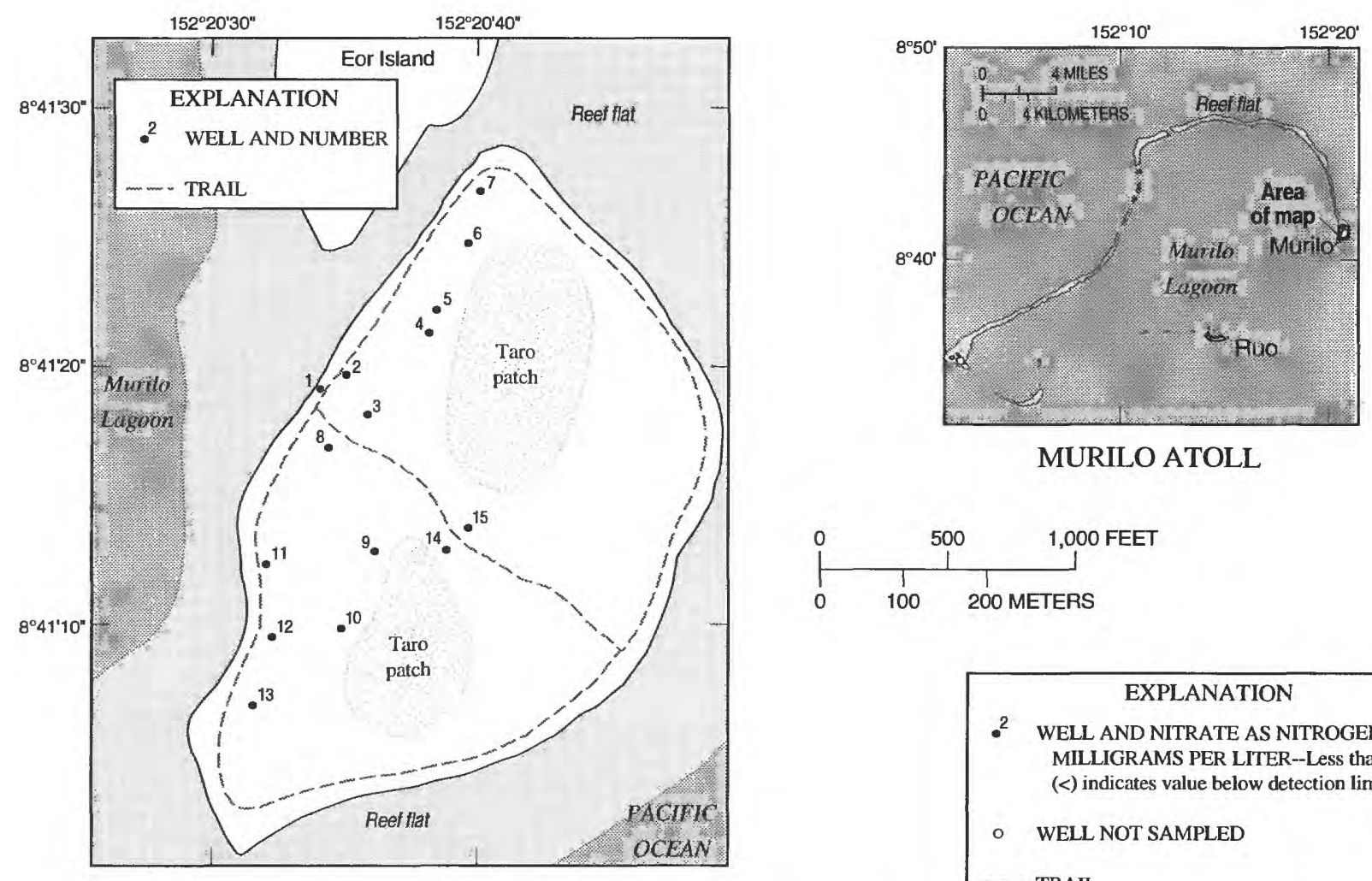

MURILO ATOLL
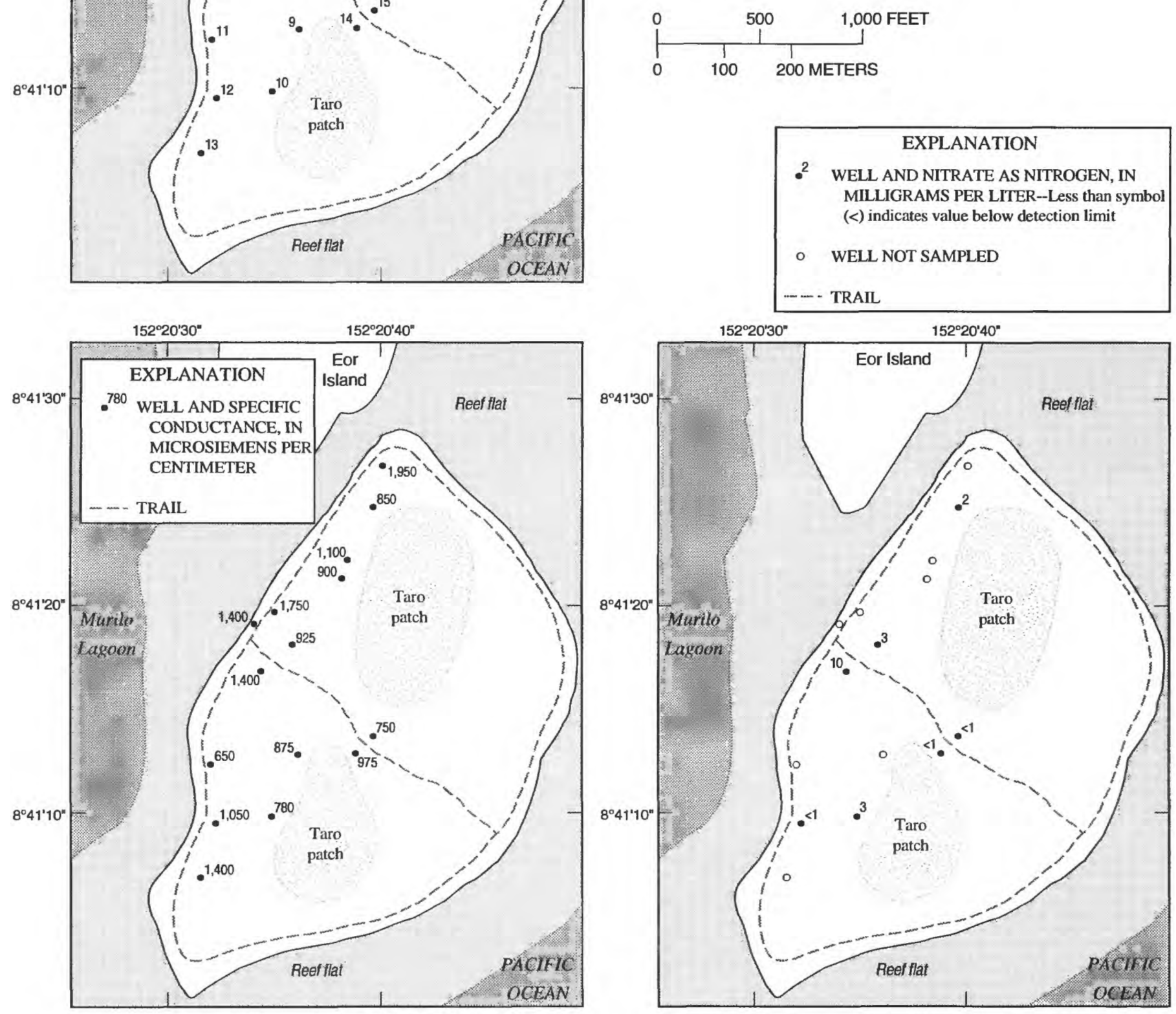

Figure 23. Well locations, specific conductance and nitrate concentrations for Murilo Island, Murilo Atoll, Hall Island Group, Chuuk State, 1984. 


\section{Ruo Island}

The population of Ruo Island at Murilo Atoll is about 298 (Truk State Office of Planning and Statistics, 1981). Well locations, specific conductance, and nitrate concentrations for Ruo Island are shown in figure 24. Selected physical and water-quality data for the 10 dug wells sampled are given in table 16 . The mean specific conductance for water from 10 wells was $1,860 \mu \mathrm{S} / \mathrm{cm}$ and ranged from 250 to $6,000 \mu \mathrm{S} / \mathrm{cm}$. On the basis of the estimated chloride concentrations from specific conductance data, water from 4 of the 10 wells sampled (wells 4, 5, 8, and 9) exceeded the WHO (1984) guideline for chloride. Four of the 10 wells sampled were field tested for nitrate concentration. Water from 2 of the 4 wells tested had nitrate concentrations of less than $1 \mathrm{mg} / \mathrm{L}$ (as $\mathrm{N}$ ); the others had concentrations of 2 and 7 $\mathrm{mg} / \mathrm{L}$ (as N).

Table 16. Selected physical and water-quality data from dug wells, March 4, 1984, Ruo Island, Murilo Atoll, Hall Island Group $\left[{ }^{\circ} \mathrm{C}\right.$, degrees Celsius; $\mu \mathrm{S} / \mathrm{cm}$, microsiemens per centimeter at $25^{\circ} \mathrm{C} ; \mathrm{Cl}$, chloride; $\mathrm{NO}_{3}$, nitrate; $\mathrm{mg} / \mathrm{L}$, milligrams per liter; --, not measured; <, less than]

\begin{tabular}{|c|c|c|c|c|c|c|c|}
\hline $\begin{array}{l}\text { Well } \\
\text { no. }\end{array}$ & Owner & $\begin{array}{c}\text { Temperature } \\
\left({ }^{\circ} \mathrm{C}\right)\end{array}$ & $\begin{array}{c}\text { Speclfic } \\
\text { conductance } \\
(\mu \mathrm{S} / \mathrm{cm})\end{array}$ & pH & $\underset{(\mathrm{mg} / \mathrm{L})}{\mathrm{Cl}}$ & $\begin{array}{l}\mathrm{NO}_{3}, \\
\text { as N } \\
\text { (mg/L) }\end{array}$ & Remarks \\
\hline 1 & Koichi & 27.5 & 1,100 & 7.4 & -- & -- & \\
\hline 2 & Tito & 27.5 & 1,150 & -- & -- & 7 & \\
\hline 3 & Ritok & 27.5 & 1,500 & -- & - & - & \\
\hline 4 & Pio & 27 & 1,550 & 6.7 & 180 & 7 & Major ion and trace element sample \\
\hline 5 & Koichi & 27.5 & 2,050 & -- & -- & -- & \\
\hline 6 & Erman & 28 & 1,400 & -- & -- & $<1$ & \\
\hline 7 & Ichiro & 27 & 1,500 & -- & -- & -- & \\
\hline 8 & David & 28.5 & 6,000 & -- & -- & -- & \\
\hline 9 & Nafer & 27 & 2,150 & -- & -- & $<1$ & \\
\hline 10 & Yoichi & 27 & 250 & -- & -- & -- & \\
\hline
\end{tabular}



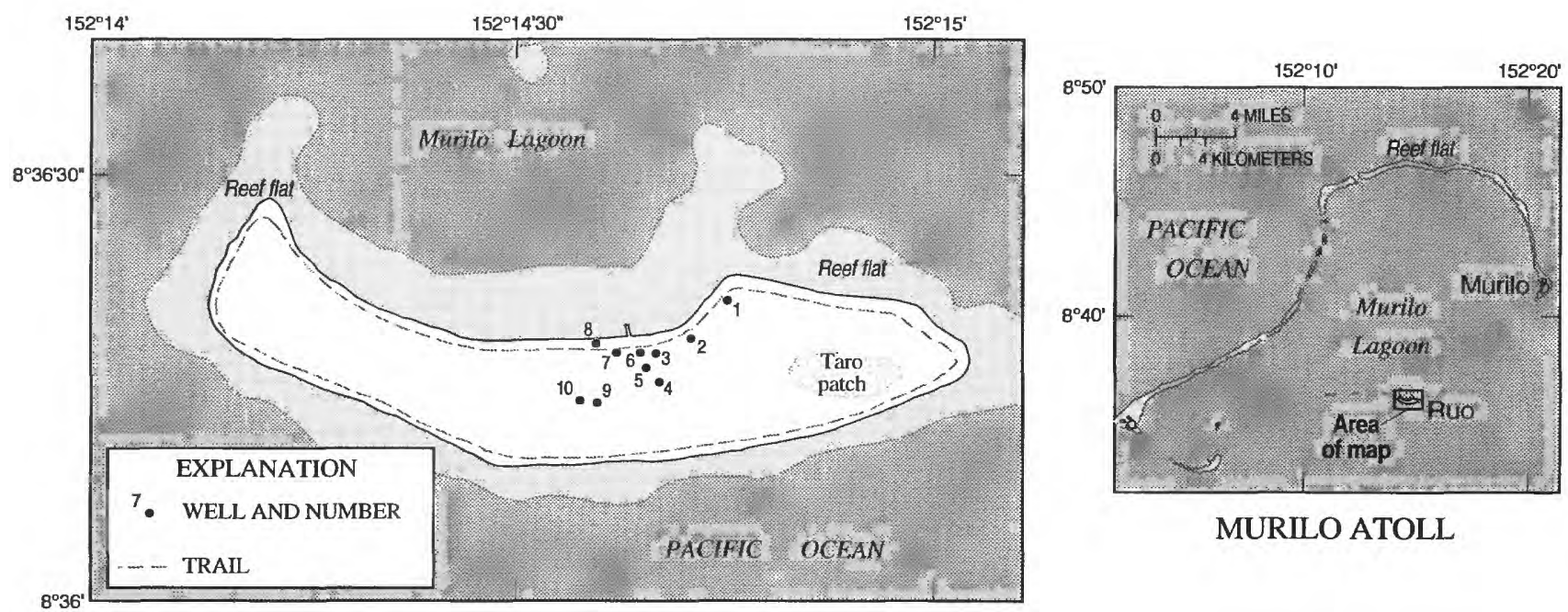

MURILO ATOLL
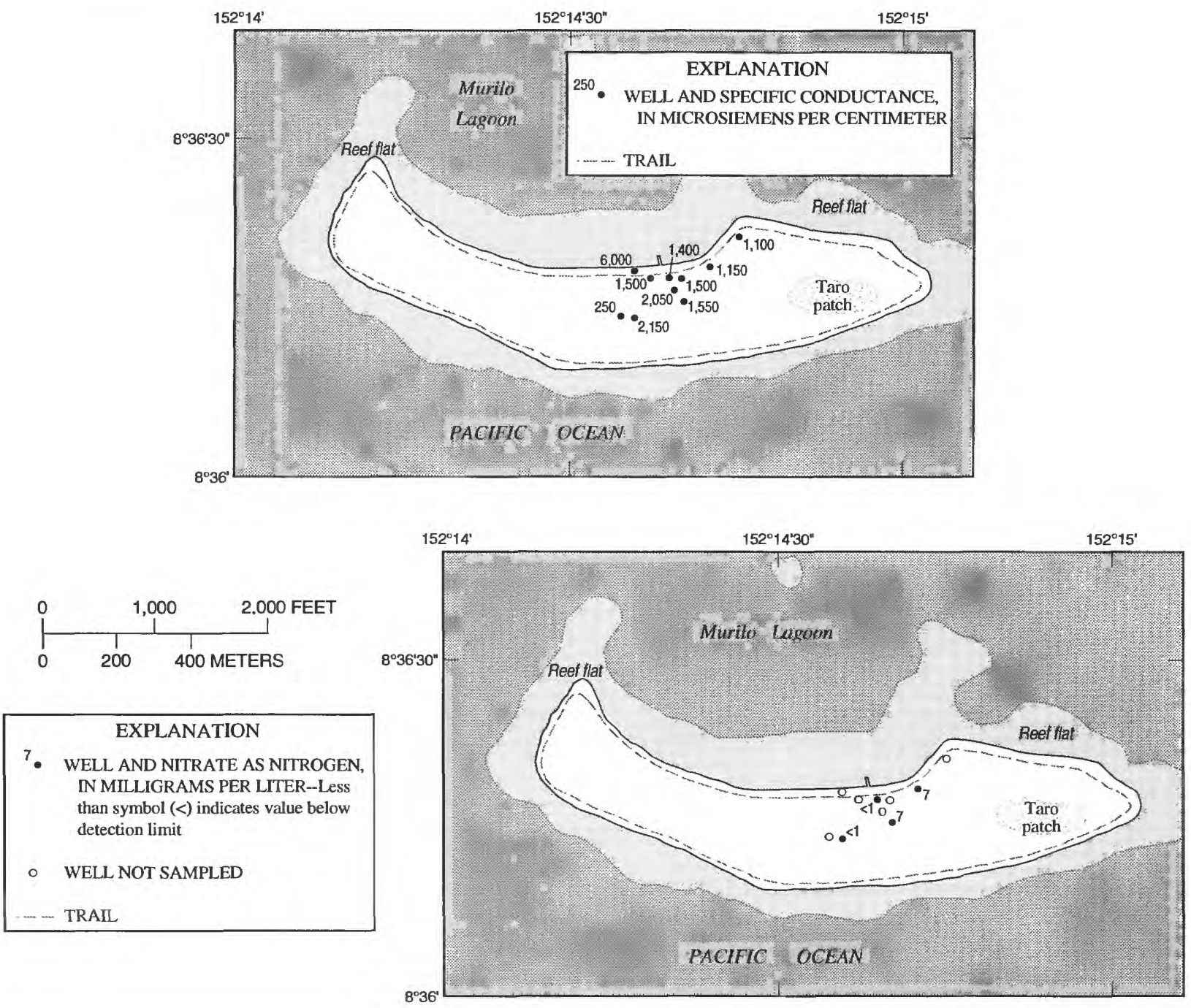

Figure 24. Well locations, specific conductance, and nitrate concentrations for Ruo Island, Murilo Atoll, Hall Island Group, Chuuk State, 1984. 


\section{Mortlock Island Group}

The inhabited islands of the Mortlock Island Group are Nema Island, Losap and Piis-Emwar Islands at Losap Atoll, Namoluk Island at Namoluk Atoll, Ettal Island at Ettal Atoll, Lukunoch and Oneop Islands at Lukunoch Atoll, and Satowan, Ta, Kuttu, and Moch Islands at Satowan Atoll (fig. 25). These islands are between 50 and $200 \mathrm{mi}$ southwest of the Chuuk Island Group. Mean values for specific conductance of ground water on each of the islands ranged between $660 \mu \mathrm{S} / \mathrm{cm}$ on Namoluk Island to 2,650 $\mathrm{S} / \mathrm{cm}$ on Nema Island. Field determinations for nitrate concentration of ground water ranged from less than $1 \mathrm{mg} / \mathrm{L}$ (as N) at selected sites on all islands to $14 \mathrm{mg} / \mathrm{L}$ (as N) on Kuttu Island. Table 17 includes physical, major-ion, and trace-element data for selected wells in the Mortlock Island Group. Specific information for each of the islands is presented below.

Table 17. Physical, major ion, and trace element data for selected wells in the Mortlock Island Group, Chuuk State $\left[{ }^{\circ} \mathrm{C}\right.$, degrees Celsius; $\mu \mathrm{S} / \mathrm{cm}$, microsiemens per centimeter at $25^{\circ} \mathrm{C}$; mg/L, milligrams per liter, $\mu \mathrm{g} / \mathrm{L}$, micrograms per liter, $\mathrm{CaCO}_{3}$, calciumcarbonate; --, not measured; <, less than]

\begin{tabular}{|c|c|c|c|c|}
\hline Property or constituent & Units & $\begin{array}{c}\text { Nema } \\
\text { well } 3 \\
03 / 03 / 85\end{array}$ & $\begin{array}{c}\text { Losap } \\
\text { well } 6 \\
03 / 06 / 85\end{array}$ & $\begin{array}{c}\text { Pils-Emwar } \\
\text { well } 9 \\
03 / 04 / 85\end{array}$ \\
\hline Specific conductance $\ldots \ldots \ldots \ldots \ldots \ldots \ldots$ & $\mu \mathrm{S} / \mathrm{cm}$ & 8,000 & 1,090 & 630 \\
\hline$\ldots \ldots \ldots \ldots \ldots \ldots \ldots \ldots$ & units & 7.3 & 7.1 & 7.1 \\
\hline 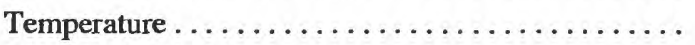 & ${ }^{\circ} \mathrm{C}$ & 27.0 & 27.0 & 27.0 \\
\hline Hardness as $\mathrm{CaCO}_{3}$ & $\mathrm{mg} / \mathrm{L}$ & - & -- & - \\
\hline Calcium, total as $\mathrm{Ca} . \ldots \ldots \ldots \ldots \ldots \ldots \ldots \ldots \ldots$ & $\mathrm{mg} / \mathrm{L}$ & 180 & 130 & 96 \\
\hline Magnesium, total as $\mathrm{Mg} \ldots \ldots \ldots \ldots \ldots \ldots$ & $\mathrm{mg} / \mathrm{L}$ & 160 & 20 & - \\
\hline Sodium, total as $\mathrm{Na} \ldots \ldots \ldots \ldots \ldots \ldots \ldots$ & $\mathrm{mg} / \mathrm{L}$ & 1,300 & 67 & 16 \\
\hline Potassium, total as $\mathrm{K} \ldots \ldots \ldots \ldots \ldots \ldots \ldots$ & $\mathrm{mg} / \mathrm{L}$ & 48 & 18 & 15 \\
\hline Alkalinity, total as $\mathrm{CaCO}_{3}$. & $\mathrm{mg} / \mathrm{L}$ & 304 & 396 & 257 \\
\hline Sulfate, dissolved as $\mathrm{SO}_{4}$ & $\mathrm{mg} / \mathrm{L}$ & 330 & 36 & 12 \\
\hline Chloride, dissolved as $\mathrm{Cl} \ldots \ldots \ldots \ldots \ldots \ldots$ & $\mathrm{mg} / \mathrm{L}$ & 2,300 & 83 & 19 \\
\hline Fluoride, total as F . . . . . . . . . . . . . & $\mathrm{mg} / \mathrm{L}$ & 0.4 & 0.3 & 0.2 \\
\hline Silica, dissolved as $\mathrm{SO}_{2} \ldots$ & $\mathrm{mg} / \mathrm{L}$ & 1.8 & 6.3 & 3.2 \\
\hline 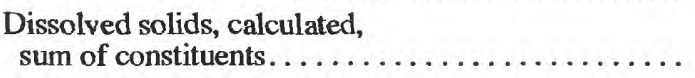 & $\mathrm{mg} / \mathrm{L}$ & 4,510 & 628 & 351 \\
\hline Aluminum, total as $\mathrm{Al} \ldots \ldots \ldots \ldots \ldots \ldots$ & $\mathrm{mg} / \mathrm{L}$ & 200 & 50 & 90 \\
\hline 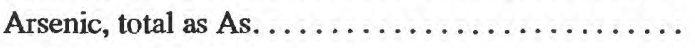 & $\mu \mathrm{g} / \mathrm{L}$ & $<1$ & 2 & $<1$ \\
\hline Barium, total as $\mathrm{Ba} \ldots \ldots \ldots \ldots \ldots \ldots \ldots$ & $\mu \mathrm{g} / \mathrm{L}$ & 200 & 200 & 200 \\
\hline Beryllium, total as Be. . . . . . . . . . . . & $\mu \mathrm{g} / \mathrm{L}$ & $<10$ & $<10$ & $<10$ \\
\hline Cadmium, total as $\mathrm{Cd} . \ldots \ldots \ldots \ldots \ldots \ldots \ldots$ & $\mu \mathrm{g} / \mathrm{L}$ & 2 & $<1$ & $<1$ \\
\hline Chromium, total as $\mathbf{C r} \ldots \ldots \ldots \ldots \ldots \ldots$ & $\mu \mathrm{g} / \mathrm{L}$ & 20 & 20 & 10 \\
\hline Cobalt, total as Co $\ldots \ldots \ldots \ldots \ldots \ldots \ldots \ldots$ & $\mu \mathrm{g} / \mathrm{L}$ & $<1$ & $<1$ & 1 \\
\hline Copper, total as $\mathrm{Cu} \quad \ldots \ldots \ldots \ldots \ldots \ldots \ldots$ & $\mu \mathrm{g} / \mathrm{L}$ & 2 & 7 & 6 \\
\hline Iron, total as $\mathrm{Fe} \ldots \ldots \ldots \ldots \ldots \ldots \ldots \ldots$ & $\mu \mathrm{g} / \mathrm{L}$ & 690 & 110 & 190 \\
\hline Iron, dissolved as Fe. . . . . . . . . . . . . & $\mu \mathrm{g} / \mathrm{L}$ & 70 & 70 & 35 \\
\hline Lead, total as $\mathrm{Pb} \ldots \ldots \ldots \ldots \ldots \ldots \ldots \ldots \ldots$ & $\mu \mathrm{g} / \mathrm{L}$ & $<1$ & $<1$ & $<1$ \\
\hline Lithium, total as Li. . . . . . . . . . . . . & $\mu \mathrm{g} / \mathrm{L}$ & 290 & 110 & 80 \\
\hline 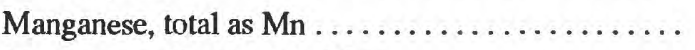 & $\mu \mathrm{g} / \mathrm{L}$ & 30 & 20 & 60 \\
\hline Manganese, dissolved as Mn . . . . . . . . . . . & $\mu \mathrm{g} / \mathrm{L}$ & 20 & 1 & 8.2 \\
\hline Mercury, total as $\mathrm{Hg} . \ldots \ldots \ldots \ldots \ldots \ldots \ldots$ & $\mu \mathrm{g} / \mathrm{L}$ & - & -- & - \\
\hline Molybdenum, total as Mo $\ldots \ldots \ldots \ldots \ldots \ldots \ldots$ & $\mu \mathrm{g} / \mathrm{L}$ & 1 & 1 & $<1$ \\
\hline Nickel, total as $\mathrm{Ni} \ldots \ldots \ldots \ldots \ldots \ldots \ldots \ldots$ & $\mu \mathrm{g} / \mathrm{L}$ & 7 & 6 & 4 \\
\hline Selenium, total as $\mathrm{Se} \ldots \ldots \ldots \ldots \ldots \ldots \ldots$ & $\mu \mathrm{g} / \mathrm{L}$ & $<1$ & $<1$ & $<1$ \\
\hline Silver, total as $\mathrm{Ag} \ldots \ldots \ldots \ldots \ldots \ldots \ldots$. & $\mu \mathrm{g} / \mathrm{L}$ & $<1$ & $<1$ & $<1$ \\
\hline Zinc, total as Zn .... & $\mu \mathrm{g} / \mathrm{L}$ & 20 & 30 & 20 \\
\hline
\end{tabular}


Table 17. Physical, major ion, and trace element data for selected wells in the Mortlock Island Group, Chuuk State --Continued

\begin{tabular}{|c|c|c|c|c|c|}
\hline Property or constituent & Units & $\begin{array}{c}\text { Namoluk } \\
\text { well } 28 \\
02 / 21 / 84\end{array}$ & $\begin{array}{c}\text { Ettal } \\
\text { well } 20 \\
02 / 19 / 84\end{array}$ & $\begin{array}{c}\text { Lukunoch } \\
\text { well } 35 \\
10 / 25 / 85\end{array}$ & $\begin{array}{c}\text { Oneop } \\
\text { well } 15 \\
10 / 24 / 85\end{array}$ \\
\hline Specific conductance................. & $\mu \mathrm{S} / \mathrm{cm}$ & 600 & 650 & 900 & 670 \\
\hline $\mathrm{pH} \ldots \ldots \ldots \ldots \ldots \ldots$ & units & 7.1 & 7.1 & 7.0 & 6.9 \\
\hline Temperature $\ldots \ldots \ldots \ldots \ldots \ldots \ldots \ldots$ & ${ }^{\circ} \mathrm{C}$ & 28.5 & 26.5 & 27.5 & 29.5 \\
\hline Hardness as $\mathrm{CaCO}_{3} \ldots$ & $\mathrm{mg} / \mathrm{L}$ & 310 & 310 & 370 & 350 \\
\hline Calcium, total as $\mathrm{Ca} \ldots \ldots \ldots \ldots \ldots \ldots$ & $\mathrm{mg} / \mathrm{L}$ & 110 & 96 & 120 & - \\
\hline Magnesium, total as $\mathrm{Mg} . \ldots \ldots \ldots \ldots \ldots$ & $\mathrm{mg} / \mathrm{L}$ & 8.6 & 16 & 17 & 11 \\
\hline Sodium, total as $\mathrm{Na} . . . \ldots$. & $\mathrm{mg} / \mathrm{L}$ & 11 & 17 & 47 & 16 \\
\hline Potassium, total as $\mathrm{K} \ldots \ldots \ldots \ldots \ldots \ldots \ldots$ & $\mathrm{mg} / \mathrm{L}$ & 1.7 & 2.9 & 6.4 & 1.1 \\
\hline Alkalinity, total as $\mathrm{CaCO}_{3}$. & $\mathrm{mg} / \mathrm{L}$ & 300 & 310 & 325 & 326 \\
\hline Sulfate, dissolved as $\mathrm{SO}_{4}$ & $\mathrm{mg} / \mathrm{L}$ & 7.9 & 11 & 25 & - \\
\hline Chloride, dissolved as $\mathrm{Cl} \ldots \ldots \ldots \ldots \ldots$ & $\mathrm{mg} / \mathrm{L}$ & 8.3 & 20 & 73 & 23 \\
\hline Fluoride, total as $\mathrm{F} . . .$. . & $\mathrm{mg} / \mathrm{L}$ & 0.3 & 1.6 & 0.3 & 0.7 \\
\hline Silica, dissolved as $\mathrm{SO}_{2} \ldots \ldots \ldots \ldots \ldots \ldots \ldots \ldots$ & $\mathrm{mg} / \mathrm{L}$ & 3.7 & 1.2 & 3.9 & 2 \\
\hline $\begin{array}{l}\text { Dissolved solids, calculated, } \\
\text { sum of constituents ................ }\end{array}$ & $\mathrm{mg} / \mathrm{L}$ & 344 & 360 & 489 & 385 \\
\hline Aluminum, total as $\mathrm{Al} \ldots \ldots \ldots \ldots \ldots \ldots$ & $\mathrm{mg} / \mathrm{L}$ & 20 & 20 & $<10$ & $<10$ \\
\hline Arsenic, total as As . . . . . . . . . . . . . & $\mu \mathrm{g} / \mathrm{L}$ & 43 & $<1$ & 1 & $<1$ \\
\hline Barium, total as $\mathrm{Ba} \ldots \ldots \ldots \ldots \ldots \ldots$ & $\mu \mathrm{g} / \mathrm{L}$ & $<100$ & $<100$ & $<100$ & $<100$ \\
\hline Beryllium, total as $\mathrm{Be} \ldots \ldots \ldots \ldots \ldots \ldots$ & $\mu \mathrm{g} / \mathrm{L}$ & $<10$ & $<10$ & $<10$ & $<10$ \\
\hline Cadmium, total as $\mathrm{Cd} \ldots \ldots \ldots \ldots \ldots \ldots$ & $\mu \mathrm{g} / \mathrm{L}$ & 5 & -- & $<1$ & $<1$ \\
\hline Chromium, total as $\mathrm{Cr} . .$. & $\mu \mathrm{g} / \mathrm{L}$ & 10 & $<10$ & $<10$ & $<10$ \\
\hline Cobalt, total as Co................. & $\mu \mathrm{g} / \mathrm{L}$ & $<1$ & $<1$ & $<1$ & $<1$ \\
\hline Copper, total as $\mathrm{Cu} \ldots \ldots \ldots \ldots \ldots \ldots \ldots$ & $\mu \mathrm{g} / \mathrm{L}$ & 6 & 6 & 1 & 5 \\
\hline Iron, total as $\mathrm{Fe} . \ldots \ldots \ldots \ldots \ldots$ & $\mu \mathrm{g} / \mathrm{L}$ & 50 & 180 & 190 & $<10$ \\
\hline Iron, dissolved as $\mathrm{Fe} \ldots \ldots \ldots \ldots \ldots \ldots \ldots$ & $\mu \mathrm{g} / \mathrm{L}$ & 21 & 34 & 23 & 6 \\
\hline Lead, total as $\mathrm{Pb} . \ldots \ldots \ldots \ldots \ldots \ldots \ldots$. & $\mu \mathrm{g} / \mathrm{L}$ & 4 & 4 & 3 & -- \\
\hline Lithium, total as $\mathrm{Li} \ldots \ldots \ldots \ldots \ldots \ldots \ldots$ & $\mu \mathrm{g} / \mathrm{L}$ & $<10$ & $<10$ & $<10$ & $<10$ \\
\hline Manganese, total as Mn. . . . . . . . . . . & $\mu \mathrm{g} / \mathrm{L}$ & 10 & 20 & 20 & 20 \\
\hline Manganese, dissolved as Mn.............. & $\mu \mathrm{g} / \mathrm{L}$ & 10 & - & 10 & 11 \\
\hline Mercury, total as $\mathrm{Hg} \ldots \ldots \ldots \ldots \ldots \ldots \ldots$ & $\mu \mathrm{g} / \mathrm{L}$ & $<0.1$ & $<0.1$ & - & - \\
\hline Molybdenum, total as Mo................ & $\mu \mathrm{g} / \mathrm{L}$ & 1 & 1 & $<1$ & 1 \\
\hline Nickel, total as $\mathrm{Ni} \ldots \ldots \ldots \ldots \ldots \ldots \ldots \ldots$ & $\mu \mathrm{g} / \mathrm{L}$ & 11 & 9 & 6 & 5 \\
\hline Selenium, total as Se . . . . . . . . . . . & $\mu \mathrm{g} / \mathrm{L}$ & $<1$ & $<1$ & $<1$ & $<1$ \\
\hline Silver, total as $\mathrm{Ag} \ldots$ & $\mu \mathrm{g} / \mathrm{L}$ & 2 & $<1$ & 1 & $<1$ \\
\hline Zinc, total as $\mathrm{Zn}$.. & $\mu \mathrm{g} / \mathrm{L}$ & 30 & 50 & 30 & 20 \\
\hline
\end{tabular}


Table 17. Physical, major ion, and trace element data for selected wells in the Mortlock Island Group, Chuuk State --Continued

\begin{tabular}{|c|c|c|c|c|c|}
\hline Property or constituent & Unlts & $\begin{array}{c}\text { Satowan } \\
\text { well } 38 \\
10 / 23 / 85\end{array}$ & $\begin{array}{c}\mathrm{Ta} \\
\text { well 18 } \\
10 / 27 / 85\end{array}$ & $\begin{array}{c}\text { Kuttu } \\
\text { well 28 } \\
02 / 17 / 84\end{array}$ & $\begin{array}{c}\text { Moch } \\
\text { well 18 } \\
02 / 18 / 84\end{array}$ \\
\hline Specific conductance......... & $\mu \mathrm{S} / \mathrm{cm}$ & -- & 860 & 750 & 800 \\
\hline $\mathrm{pH} \ldots \ldots \ldots \ldots$ & units & 7.1 & 7.1 & 7.4 & 7.3 \\
\hline Temperature $\ldots \ldots \ldots \ldots$ & ${ }^{\circ} \mathrm{C}$ & 27.5 & 27.5 & 27.0 & 27.0 \\
\hline Hardness as $\mathrm{CaCO}_{3}$. & $\mathrm{mg} / \mathrm{L}$ & 200 & 320 & 270 & 310 \\
\hline Calcium, total as $\mathrm{Ca} \ldots \ldots$. & $\mathrm{mg} / \mathrm{L}$ & 72 & 87 & 83 & 100 \\
\hline Magnesium, total as $\mathrm{Mg} \ldots \ldots \ldots \ldots \ldots \ldots$ & $\mathrm{mg} / \mathrm{L}$ & 5.8 & 24 & 15 & 15 \\
\hline Sodium, total as Na ..................... & $\mathrm{mg} / \mathrm{L}$ & 14 & 65 & 43 & 44 \\
\hline Potassium, total as $\mathrm{K} . \ldots \ldots \ldots \ldots \ldots \ldots \ldots \ldots$ & $\mathrm{mg} / \mathrm{L}$ & 0.7 & 2.7 & 7.5 & 4 \\
\hline Alkalinity, total as $\mathrm{CaCO}_{3}$. & $\mathrm{mg} / \mathrm{L}$ & 194 & 276 & 268 & 316 \\
\hline Sulfate, dissolved as $\mathrm{SO}_{4}$ & $\mathrm{mg} / \mathrm{L}$ & 19 & 28 & 29 & 32 \\
\hline Chloride, dissolved as $\mathrm{Cl} \ldots \ldots \ldots \ldots \ldots \ldots$ & $\mathrm{mg} / \mathrm{L}$ & 11 & 100 & 45 & 43 \\
\hline Fluoride, total as $\mathrm{F} \ldots \ldots \ldots \ldots \ldots \ldots \ldots \ldots$ & $\mathrm{mg} / \mathrm{L}$ & 0.3 & 0.4 & 0.4 & 0.2 \\
\hline Silica, dissolved as $\mathrm{SO}_{2}$. & $\mathrm{mg} / \mathrm{L}$ & 1.7 & 0.4 & 4.4 & 3.3 \\
\hline $\begin{array}{l}\text { Dissolved solids, calculated, } \\
\text { sum of constituents ....... }\end{array}$ & $\mathrm{mg} / \mathrm{L}$ & 243 & 473 & 421 & 458 \\
\hline Aluminum, total as Al...... & $\mathrm{mg} / \mathrm{L}$ & $<10$ & 10 & 30 & 20 \\
\hline Arsenic, total as As...... & $\mu \mathrm{g} / \mathrm{L}$ & $<1$ & $<1$ & 1 & 1 \\
\hline Barium, total as $\mathrm{Ba} \ldots \ldots \ldots \ldots \ldots \ldots \ldots \ldots$ & $\mu \mathrm{g} / \mathrm{L}$ & $<100$ & $<100$ & $<100$ & $<100$ \\
\hline Beryllium, total as $\mathrm{Be} \ldots \ldots \ldots \ldots \ldots \ldots \ldots$ & $\mu \mathrm{g} / \mathrm{L}$ & $<10$ & $<10$ & $<10$ & $<10$ \\
\hline Cadmium, total as $\mathrm{Cd} \ldots \ldots \ldots \ldots \ldots \ldots \ldots$ & $\mu \mathrm{g} / \mathrm{L}$ & 1 & 1 & 3 & 1 \\
\hline Chromium, total as $\mathrm{Cr} . \ldots \ldots \ldots \ldots \ldots \ldots$ & $\mu \mathrm{g} / \mathrm{L}$ & $<10$ & $<10$ & $<10$ & $<10$ \\
\hline Cobalt, total as Co.................... & $\mu \mathrm{g} / \mathrm{L}$ & 1 & $<1$ & $<1$ & 2 \\
\hline Copper, total as $\mathrm{Cu} . \ldots \ldots \ldots \ldots \ldots \ldots \ldots$. & $\mu \mathrm{g} / \mathrm{L}$ & -- & 13 & 3 & 5 \\
\hline Iron, total as $\mathrm{Fe} \ldots \ldots \ldots \ldots \ldots \ldots \ldots \ldots$ & $\mu \mathrm{g} / \mathrm{L}$ & 250 & 210 & 40 & 100 \\
\hline Iron, dissolved as $\mathrm{Fe} \ldots \ldots \ldots \ldots \ldots \ldots \ldots$ & $\mu \mathrm{g} / \mathrm{L}$ & 24 & 40 & 26 & 37 \\
\hline Lead, total as $\mathrm{Pb} \ldots \ldots \ldots \ldots \ldots \ldots$ & $\mu \mathrm{g} / \mathrm{L}$ & 3 & 7 & 3 & 3 \\
\hline Lithium, total as $\mathrm{Li} \ldots \ldots \ldots \ldots \ldots \ldots \ldots$ & $\mu g / L$ & $<10$ & $<10$ & $<10$ & $<10$ \\
\hline Manganese, total as Mn.................. & $\mu \mathrm{g} / \mathrm{L}$ & 20 & 20 & 57 & 46 \\
\hline Manganese, dissolved as Mn. .............. & $\mu \mathrm{g} / \mathrm{L}$ & 9 & 9 & 57 & 46 \\
\hline Mercury, total as $\mathrm{Hg} \ldots \ldots \ldots \ldots \ldots \ldots \ldots \ldots$ & $\mu \mathrm{g} / \mathrm{L}$ & -- & - & 0.1 & $<0.1$ \\
\hline Molybdenum, total as Mo................. & $\mu \mathrm{g} / \mathrm{L}$ & $<1$ & $<1$ & 1 & 1 \\
\hline Nickel, total as $\mathrm{Ni} \ldots \ldots \ldots \ldots \ldots \ldots \ldots \ldots$ & $\mu \mathrm{g} / \mathrm{L}$ & 6 & 3 & 8 & 13 \\
\hline Selenium, total as Se.$\ldots \ldots \ldots \ldots \ldots \ldots \ldots$ & $\mu \mathrm{g} / \mathrm{L}$ & $<1$ & $<1$ & $<1$ & $<1$ \\
\hline Silver, total as $\mathrm{Ag} \ldots \ldots \ldots \ldots \ldots \ldots \ldots \ldots$ & $\mu \mathrm{g} / \mathrm{L}$ & $<1$ & $<1$ & $<1$ & $<1$ \\
\hline Zinc, total as $\mathrm{Zn} \ldots \ldots \ldots \ldots \ldots \ldots \ldots \ldots$ & $\mu \mathrm{g} / \mathrm{L}$ & 1,000 & 400 & 170 & 70 \\
\hline
\end{tabular}




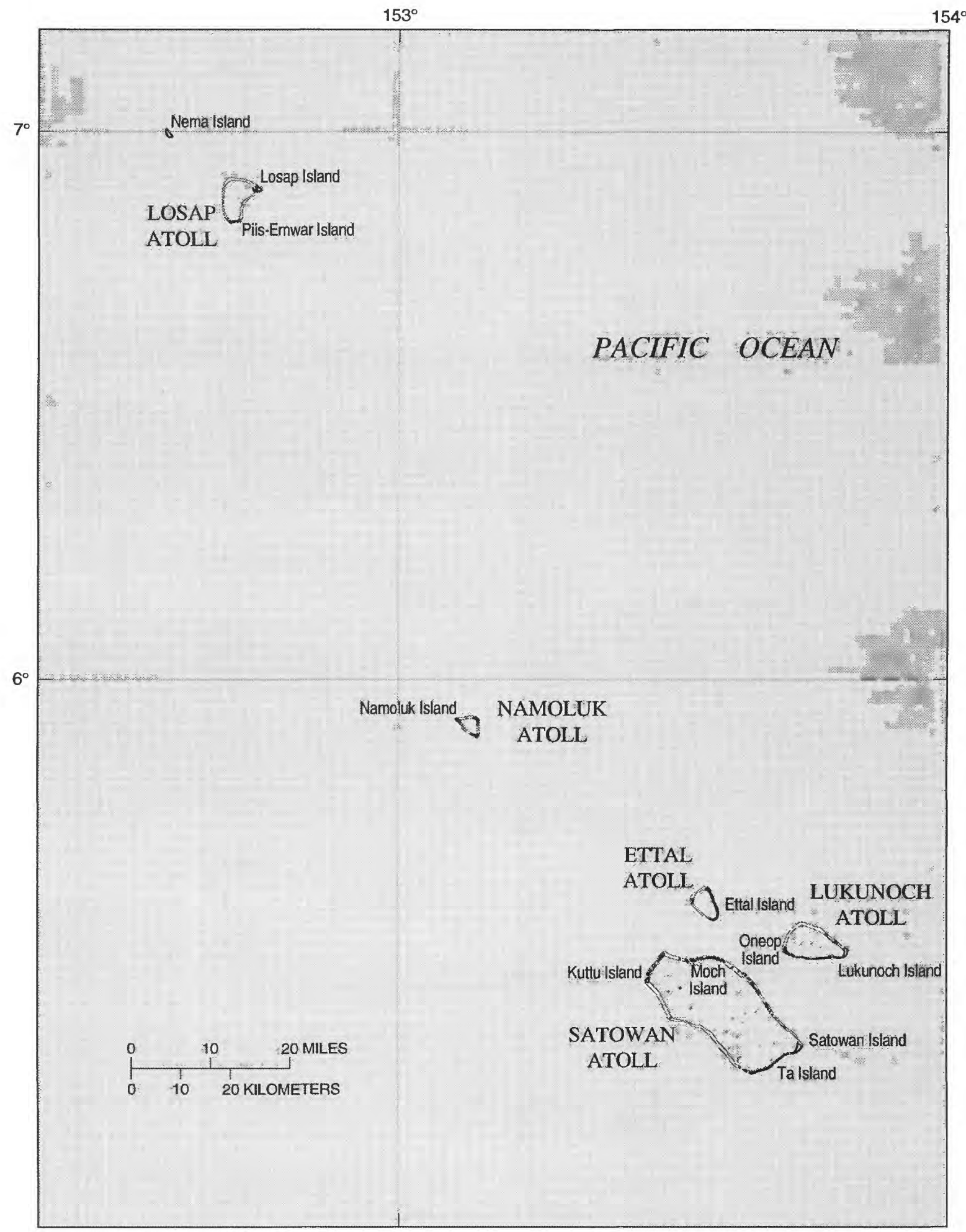

Figure 25. Mortlock Island Group, Chuuk State, Federated States of Micronesia. 


\section{Nema Island}

The population of Nema Island is about 1,021 (Truk State Office of Planning and Statistics, 1981). Well locations, specific conductance, and nitrate concentrations for Nema Island are shown in figure 26. Selected physical and water-quality data for the 11 dug wells and 1 driven well sampled are given in table 18 . The mean specific conductance for water from 12 wells was $2,650 \mu \mathrm{S} / \mathrm{cm}$ and ranged from 750 to $8,000 \mu \mathrm{S} / \mathrm{cm}$. On the basis of the estimated chloride concentrations from specific conductance data, water from 4 of 12 wells (wells 1, 2, 3, and 6) exceeded the WHO (1984) guideline for chloride. Six of the 12 wells sampled were field tested for nitrate concentration. Water from half of the wells tested had nitrate concentrations of less than 1 $\mathrm{mg} / \mathrm{L}$ (as $\mathrm{N}$ ); the remaining samples had concentrations ranging from 1 to $3 \mathrm{mg} / \mathrm{L}$ (as $\mathrm{N}$ ).

Table 18. Selected physical and water-quality data from dug and driven wells, March 3, 4, and 7, 1984, Nema Island, Mortlock Island Group

$\left[{ }^{\circ} \mathrm{C}\right.$, degrees Celsius; $\mu \mathrm{S} / \mathrm{cm}$, microsiemens per centimeter at $25^{\circ} \mathrm{C}$; $\mathrm{Cl}$, chloride; $\mathrm{NO}_{3}$, nitrate; $\mathrm{mg} / \mathrm{L}$, milligrams per liter; --, not measured; <, less than]

\begin{tabular}{|c|c|c|c|c|c|c|c|}
\hline $\begin{array}{l}\text { Well } \\
\text { no. }\end{array}$ & Owner & $\begin{array}{c}\text { Temperature } \\
\left({ }^{\circ} \mathbf{C}\right)\end{array}$ & $\begin{array}{c}\text { Specific } \\
\text { conductance } \\
(\mu \mathrm{S} / \mathrm{cm})\end{array}$ & pH & $\begin{array}{c}\mathrm{Cl} \\
(\mathrm{mg} / \mathrm{L})\end{array}$ & $\begin{array}{c}\mathrm{NO}_{3} \\
\text { as } \mathrm{N} \\
(\mathrm{mg} / \mathrm{L})\end{array}$ & Remarks \\
\hline 1 & Raeol & 27 & 2,610 & $\ldots$ & -- & $<1$ & \\
\hline 3 & Kerad & 27 & 8,000 & 7.3 & 2,300 & 2 & Major trace element sample \\
\hline 4 & Kirsina & - & - & -- & -- & - & \\
\hline 6 & Asika & 26.5 & 6,200 & - & -- & - & \\
\hline 7 & Manil & 26 & 855 & - & -- & - & \\
\hline 8 & Ngas & 26.5 & 830 & - & -- & $<1$ & \\
\hline 9 & Martin & 26.5 & 750 & -- & 21 & $<1$ & \\
\hline
\end{tabular}



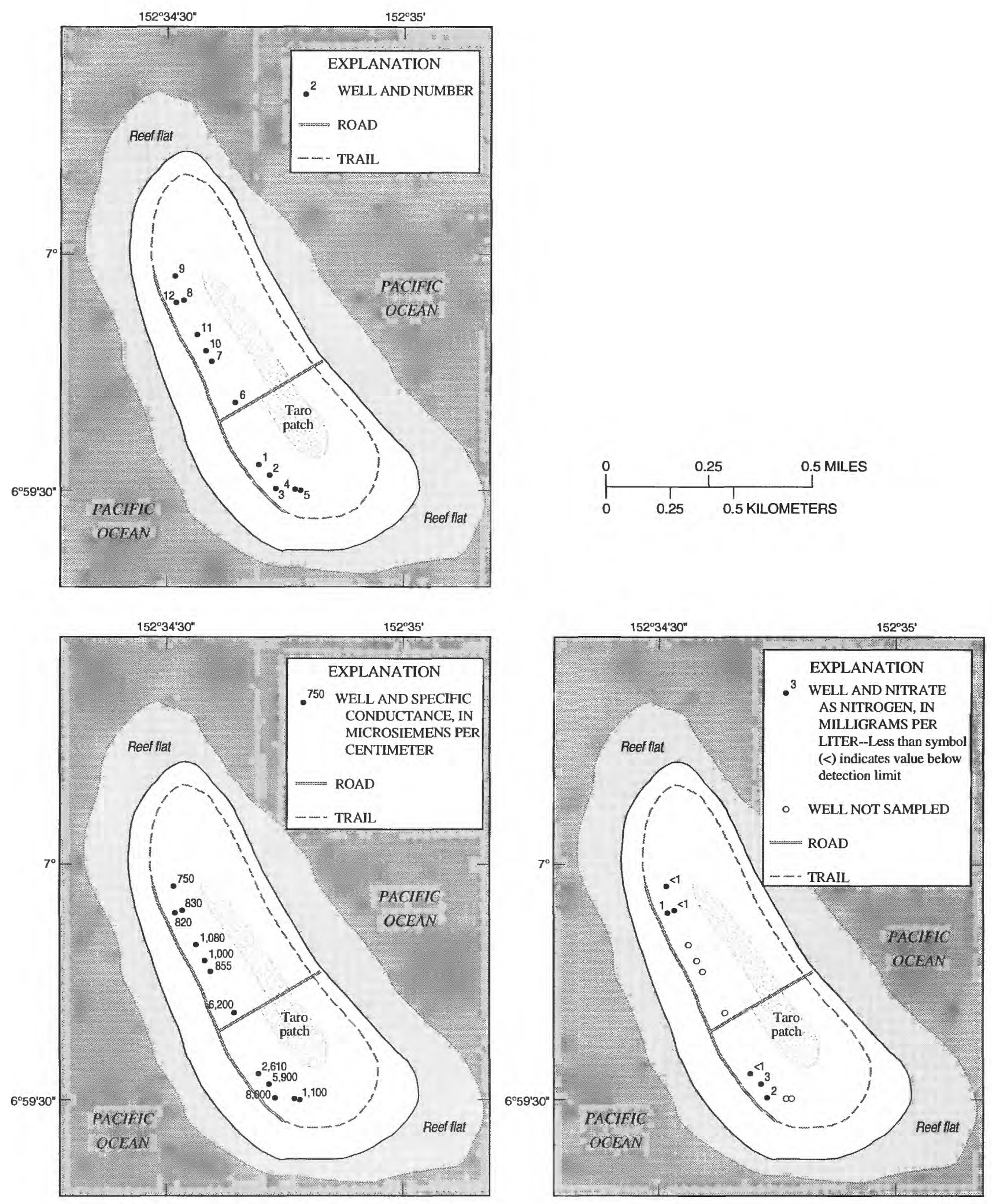

Figure 26. Well locations, specific conductance, and nitrate concentrations for Nema Island, Mortlock Island Group, Chuuk State, 1985. 


\section{Losap Island}

The population of Losap Island at Losap Atoll is about 587 (Truk State Office of Planning and Statistics, 1981). Well locations, specific conductance, and nitrate concentrations for Losap Island are shown in figure 27. Selected physical and water-quality data for the 23 dug wells and 1 driven well sampled are given in table 19. The mean specific conductance for water from 24 wells was $1,260 \mu \mathrm{S} / \mathrm{cm}$ and ranged from 620 to $3,700 \mu \mathrm{S} / \mathrm{cm}$.
On the basis of the estimated chloride concentrations from specific conductance data, water from 6 of the 24 wells sampled (wells 4, 5, 9, 11, 12, and 23) exceeded the WHO (1984) guideline for chloride. Twenty-two of the 24 wells sampled were field tested for nitrate concentration. Water from slightly more than half the wells tested had nitrate concentrations of less than $1 \mathrm{mg} / \mathrm{L}$ (as $\mathrm{N})$; the remaining samples had concentrations ranging from 1 to $6 \mathrm{mg} / \mathrm{L}$ (as $\mathrm{N}$ ).

Table 19. Selected physical and water-quality data from dug and driven wells, March 6, 1984, Losap Island, Losap Atoll, Mortlock Island Group

$\left[{ }^{\circ} \mathrm{C}\right.$, degrees Celsius; $\mu \mathrm{S} / \mathrm{cm}$, microsiemens per centimeter at $25^{\circ} \mathrm{C} ; \mathrm{Cl}$, chloride; $\mathrm{NO}_{3}$, nitrate; $\mathrm{mg} / \mathrm{L}$, milligrams per liter; --, not measured; <, less than]

\begin{tabular}{|c|c|c|c|c|c|c|c|}
\hline $\begin{array}{l}\text { Well } \\
\text { no. }\end{array}$ & Owner & $\begin{array}{c}\text { Temperature } \\
\left({ }^{\circ} \mathrm{C}\right)\end{array}$ & $\begin{array}{c}\text { Speciflc } \\
\text { conductance } \\
(\mu \mathrm{S} / \mathrm{cm})\end{array}$ & pH & $\begin{array}{c}\mathrm{Cl} \\
\text { (mg/L) }\end{array}$ & $\begin{array}{c}\mathrm{NO}_{3}, \\
\text { as N } \\
\text { (mg/L) }\end{array}$ & Remarks \\
\hline 1 & Takis & 27 & 910 & - & $\overline{--}$ & $<1$ & \\
\hline 3 & Soiakim & 27 & 620 & -- & - & $<1$ & \\
\hline 4 & Wesne & 27 & 3,700 & - & 880 & 3 & \\
\hline 7 & Samuel & 27 & 900 & -- & - & 2 & \\
\hline 8 & Sasta & 27 & 1,070 & - & - & 6 & \\
\hline 9 & Son & 27.5 & 2,200 & - & 430 & 2 & \\
\hline 10 & Anton & 26.5 & 1,300 & - & - & 1 & \\
\hline 15 & Tolove & 26.5 & 650 & -- & -- & $<1$ & \\
\hline 16 & Ichio & 27 & 850 & - & -- & $<1$ & \\
\hline 17 & Atarino & 27 & 790 & - & - & -- & \\
\hline 18 & Sachuo & 27 & 730 & - & - & $<1$ & \\
\hline 19 & Taini & 26 & 980 & - & - & $<1$ & \\
\hline 20 & Sincho & 27 & 780 & - & - & $<1$ & \\
\hline 21 & Mathew & 27 & 850 & - & - & $<1$ & \\
\hline 22 & Tupun & 27 & 1,250 & - & -- & $<1$ & \\
\hline 23 & David & 26.5 & 3,000 & $\ldots$ & 700 & $<1$ & \\
\hline
\end{tabular}



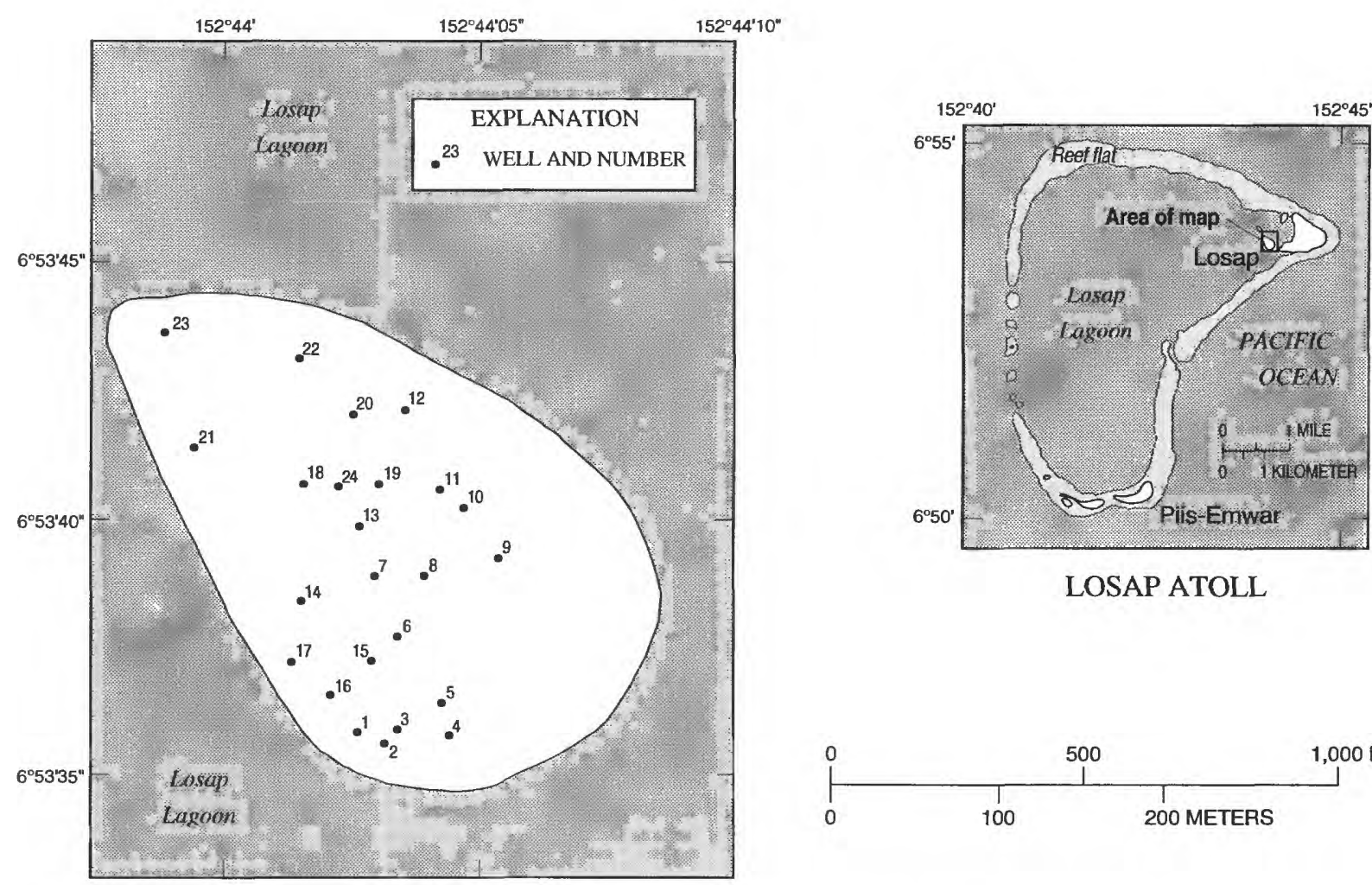

LOSAP ATOLL
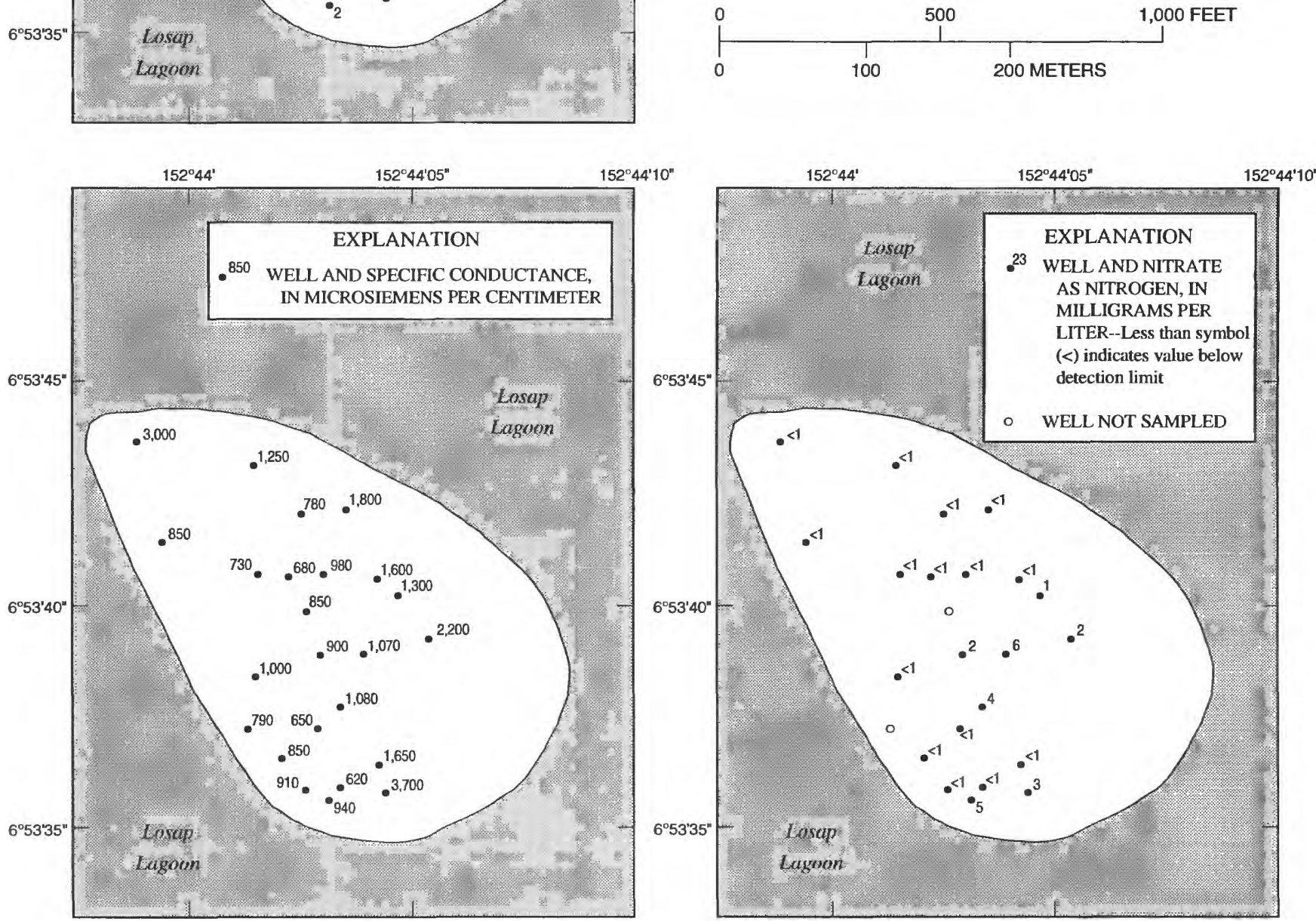

Figure 27. Well locations, specific conductance, and nitrate concentrations for Losap Island, Losap Atoll, Mortlock Island Group, Chuuk State, 1985. 


\section{Piis-Emwar Island}

The population of Piis-Emwar Island at Losap Atoll is about 240 (Truk State Office of Planning and Statistics, 1981). Well locations, specific conductance, and nitrate concentrations for Piis-Emwar Island are shown in figure 28 . Selected physical and water-quality data for the 18 dug wells, 1 driven well, and 3 taro patch sites sampled are given in table 20 . The mean specific conductance for water from 19 wells was $940 \mu \mathrm{S} / \mathrm{cm}$ and ranged from 560 to $1,700 \mu \mathrm{S} / \mathrm{cm}$. The highest mea- sured value of specific conductance $(4,000 \mu \mathrm{S} / \mathrm{cm})$ was for a water sample collected from a taro patch at the northeast end of the island. On the basis of the estimated chloride concentrations from specific conductance data, water from 1 of the 19 wells sampled (well 14) exceeded the WHO (1984) guideline for chloride. Fifteen of the 19 wells sampled were field tested for nitrate concentration. Water from 12 of the 15 wells tested had nitrate concentrations of less than $1 \mathrm{mg} / \mathrm{L}$ (as N); the others had concentrations ranging from 2 to $4 \mathrm{mg} / \mathrm{L}$ (as N).

Table 20. Selected physical and water-quality data from dug and driven wells, March 4-5, 1984, Piis-Emwar Island, Losap Atoll, Mortlock Island Group

$\left[{ }^{\circ} \mathrm{C}\right.$, degrees Celsius; $\mu \mathrm{S} / \mathrm{cm}$, microsiemens per centimeter at $25^{\circ} \mathrm{C} ; \mathrm{Cl}$, chloride; $\mathrm{NO}_{3}$, nitrate; $\mathrm{mg} / \mathrm{L}$, milligrams per liter; --, not measured; <, less than]

\begin{tabular}{|c|c|c|c|c|c|c|c|}
\hline $\begin{array}{c}\text { Well no. } \\
\text { or sampling } \\
\text { sites }\end{array}$ & Owner & $\begin{array}{c}\text { Temperature } \\
\left({ }^{\circ} \mathrm{C}\right)\end{array}$ & $\begin{array}{c}\text { Specific } \\
\text { conductance } \\
(\mu S / \mathrm{cm})\end{array}$ & pH & $\underset{(\mathrm{mg} / \mathrm{L})}{\mathrm{Cl}}$ & $\begin{array}{c}\mathrm{NO}_{3}, \\
\text { as } \mathrm{N} \\
\text { (mg/L) }\end{array}$ & Pemarks \\
\hline 1 & Kisino & 27 & 940 & - & -- & - & \\
\hline 3 & Onna & 27.5 & 760 & - & - & $<1$ & \\
\hline 4 & Tea & 27 & 1,150 & - & 130 & $<1$ & \\
\hline 7 & Reit & 28 & 855 & -- & 92 & $<1$ & \\
\hline 8 & Sachio & 26.5 & 700 & -- & -- & $<1$ & \\
\hline 9 & Kiomasa & 27 & 630 & -- & 19 & 4 & Major ion and trace element sample \\
\hline 10 & Herpis & 27.5 & 560 & -- & -- & $<1$ & \\
\hline 14 & Tony & 27 & 1,700 & -- & 270 & $<1$ & \\
\hline 15 & Robert & 26.5 & 1,300 & - & -- & $<1$ & \\
\hline 16 & Ana & 26 & 810 & -- & -- & $<1$ & \\
\hline 17 & Satachy & 26 & 740 & - & $-\infty$ & -- & \\
\hline 18 & Pesa & 26.5 & 930 & - & -- & - & \\
\hline 19 & Municipal & 27 & 660 & -- & 39 & - & Installed $11 / 4$ " well point with hand pump \\
\hline Taro patch 1 & Komet & 27 & 3,700 & -- & -- & -- & \\
\hline Taro patch 2 & & 27 & 1,380 & -- & -- & - & \\
\hline Taro patch 3 & Dochiy & 27.5 & 4,000 & -- & - & -- & \\
\hline
\end{tabular}



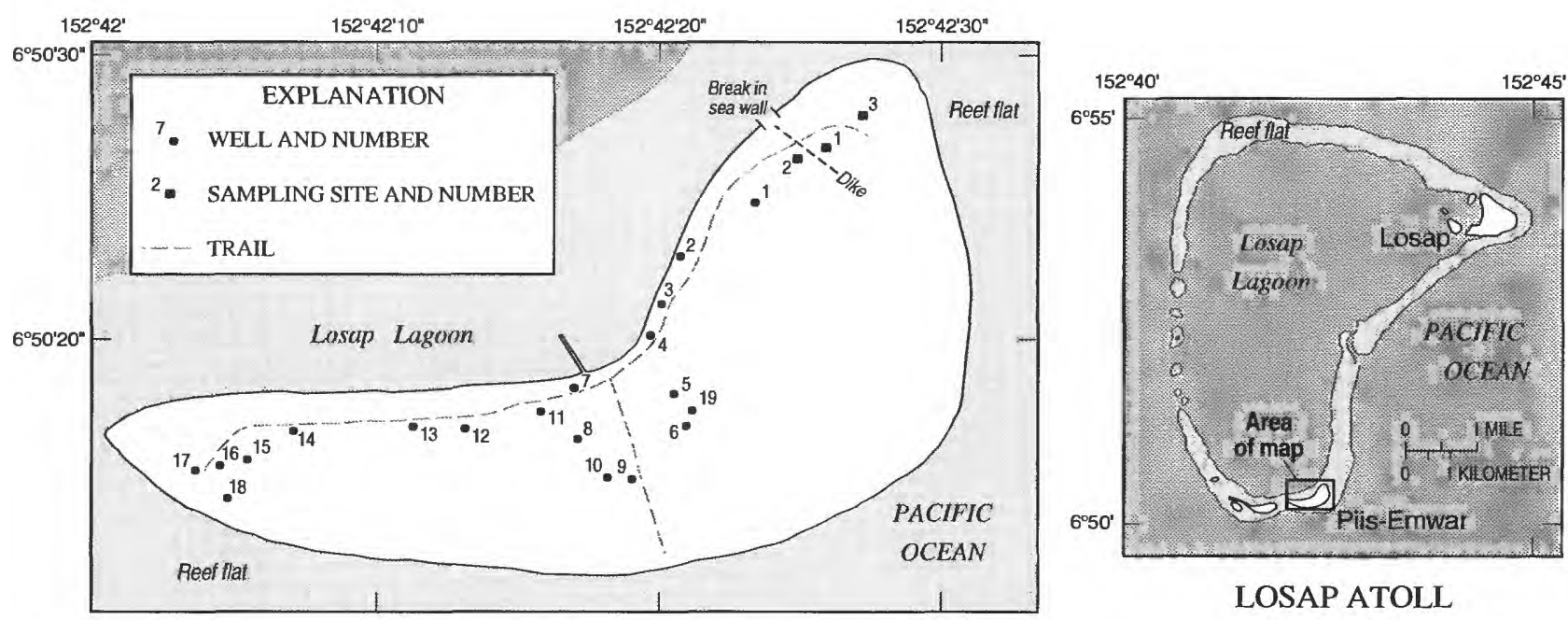

LOSAP ATOLL
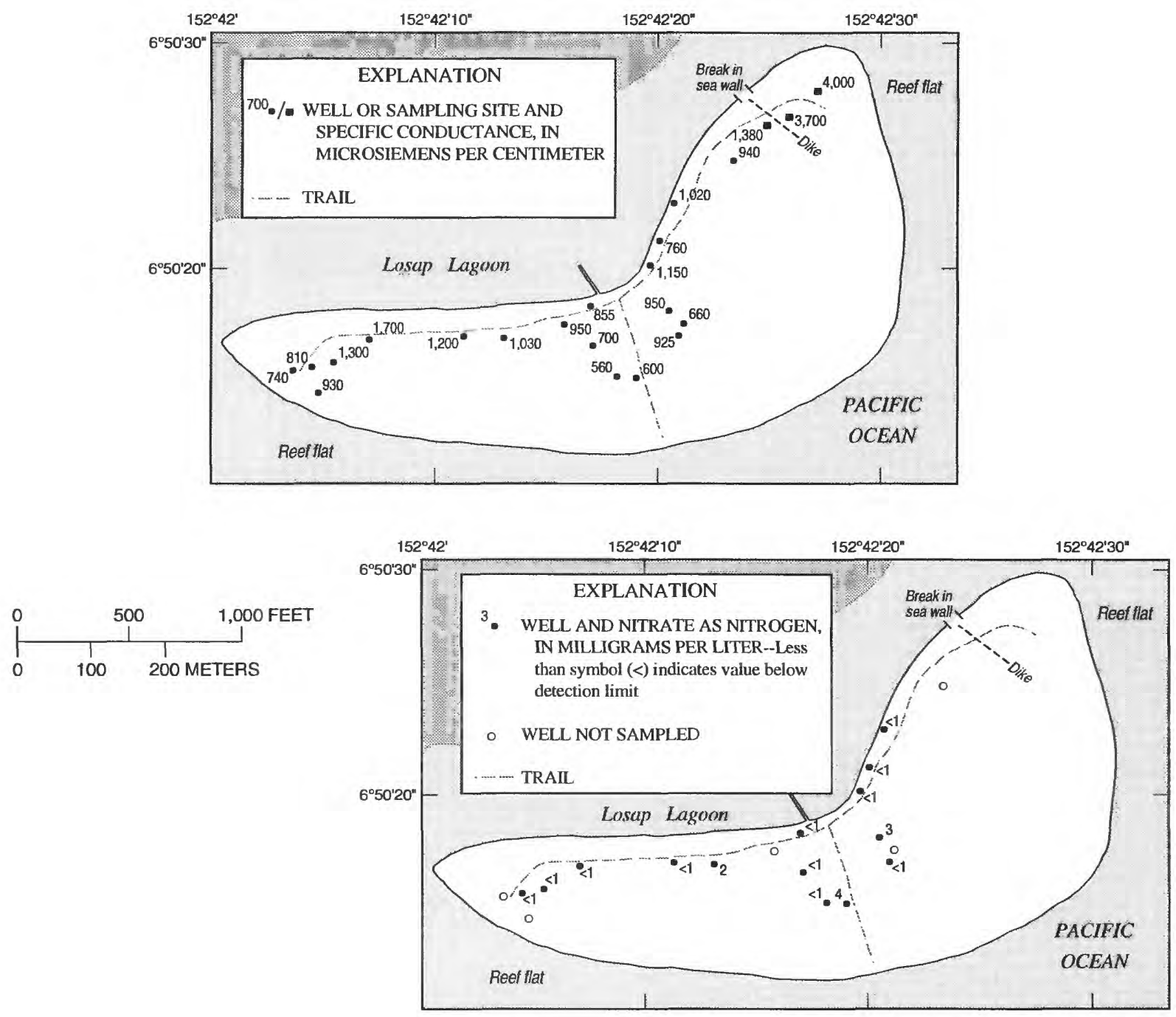

Figure 28. Well locations, specific conductance, and nitrate concentrations for Piis-Emwar Island, Losap Atoll, Mortlock Island Group, Chuuk State, 1985. 


\section{Namoluk Island}

The population of Namoluk Island at Namoluk Atoll is about 329 (Truk State Office of Planning and Statistics, 1981). Well locations, specific conductance, and nitrate concentrations for Namoluk Island are shown in figure 29 . Selected physical and water-quality data for the 28 dug wells and 1 driven well sampled are given in table 21 . The mean specific conductance for water from 29 wells was $660 \mu \mathrm{S} / \mathrm{cm}$ and ranged from 300 to $1,300 \mu \mathrm{S} / \mathrm{cm}$. On the basis of the estimated chloride concentrations from specific conductance data, none of the water samples exceeded the WHO (1984) guideline for chloride. Eleven of the 29 wells sampled were field tested for nitrate concentration. Water from 9 of the 11 wells tested had nitrate concentrations of less than $1 \mathrm{mg} / \mathrm{L}$ (as $\mathrm{N}$ ); the remaining samples had concentrations ranging from 3 to $9 \mathrm{mg} / \mathrm{L}$ (as $\mathrm{N}$ ).

Table 21. Selected physical and water-quality data from dug and driven wells, February 21, 1984, Namoluk Island, Namoluk Atoll, Mortlock Island Group

$\left[{ }^{\circ} \mathrm{C}\right.$, degrees Celsius; $\mu \mathrm{S} / \mathrm{cm}$, microsiemens per centimeter at $25^{\circ} \mathrm{C} ; \mathrm{Cl}$, chloride; $\mathrm{NO}_{3}$, nitrate; $\mathrm{mg} / \mathrm{L}$, milligrams per liter, --, not measured; <, less than]

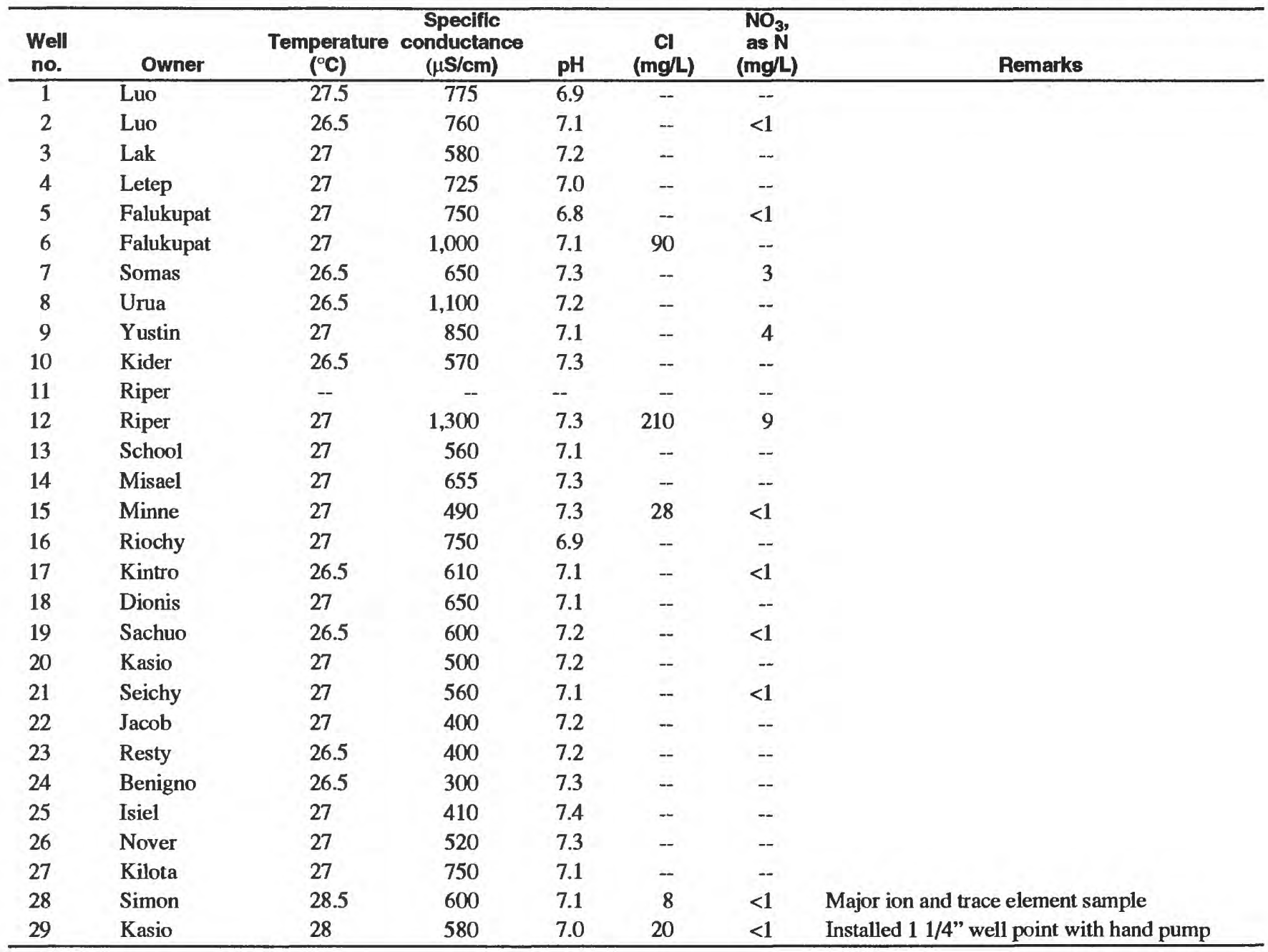



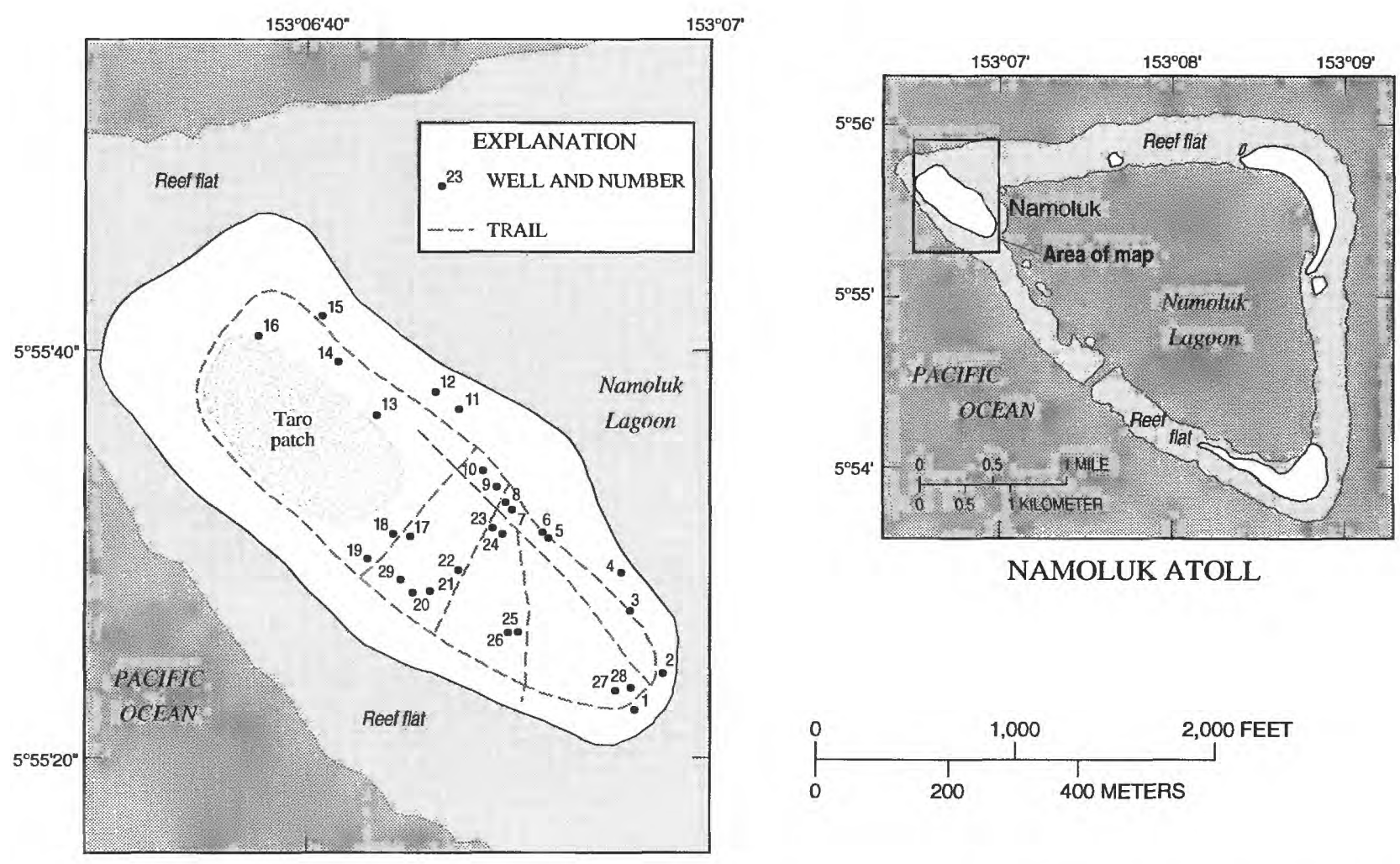

NAMOLUK ATOLL
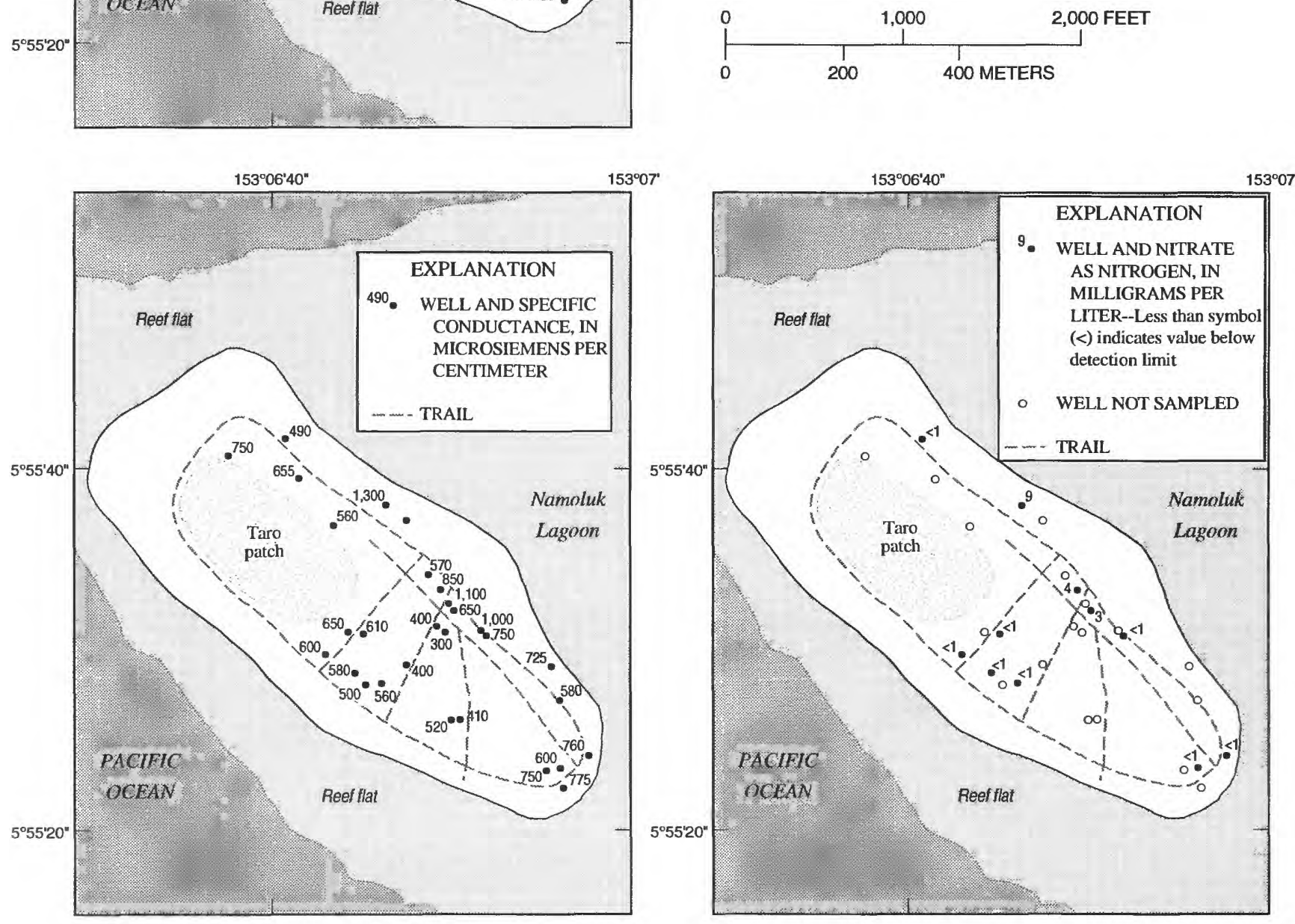

Figure 29. Well locations, specific conductance, and nitrate concentrations for Namoluk Island, Namoluk Atoll, Mortlock Island Group, Chuuk State, 1984. 


\section{Ettal Island}

The population of Ettal Island at Ettal Atoll is about 440 (Truk State Office of Planning and Statistics, 1981). Well locations, specific conductance, and nitrate concentrations for Ettal Island are shown in figure 30. Selected physical and water-quality data for the $38 \mathrm{dug}$ wells sampled are given in table 22 . The mean specific conductance for water from 38 wells was $750 \mu \mathrm{S} / \mathrm{cm}$ and ranged from 360 to $1,330 \mu \mathrm{S} / \mathrm{cm}$. On the basis of the estimated chloride concentrations from specific conductance data, none of the water samples exceeded the WHO (1984) guideline for chloride. Six of the 38 wells sampled were field tested for nitrate concentration. Water from 4 of the 6 wells tested had nitrate concentrations of less than $1 \mathrm{mg} / \mathrm{L}$ (as $\mathrm{N}$ ); the remaining samples had concentrations of 3 and $4 \mathrm{mg} / \mathrm{L}$ (as $\mathrm{N}$ ).

Table 22. Selected physical and water-quality data from dug wells, February 19, 1984, Ettal Island, Ettal Atoll, Mortlock Island Group

$\left[{ }^{\circ} \mathrm{C}\right.$, degrees Celsius; $\mu \mathrm{S} / \mathrm{cm}$, microsiemens per centimeter at $25^{\circ} \mathrm{C} ; \mathrm{Cl}$, chloride; $\mathrm{NO}_{3}$, nitrate; $\mathrm{mg} / \mathrm{L}$, milligrams per liter; --, not measured; <, less than]

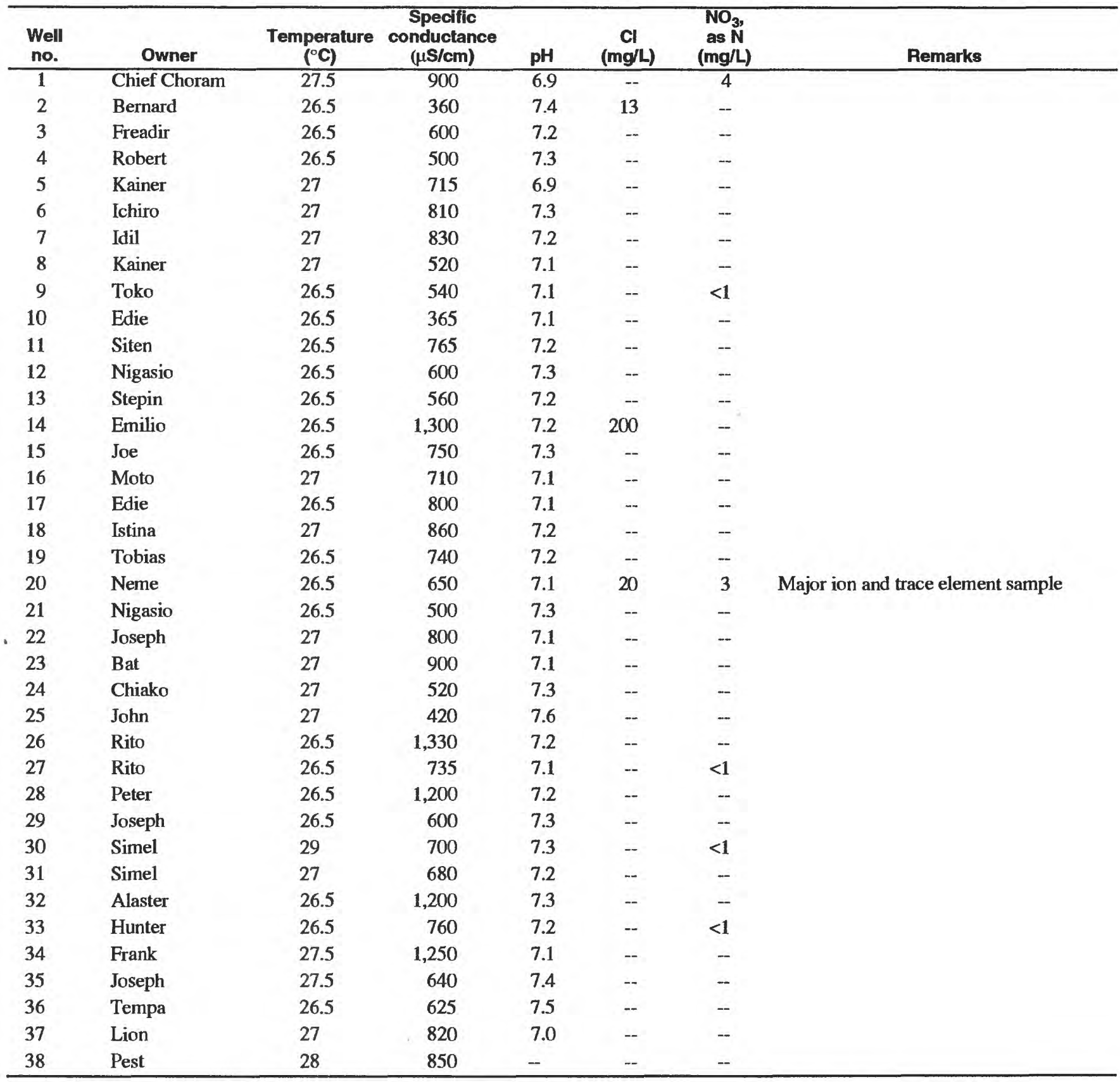



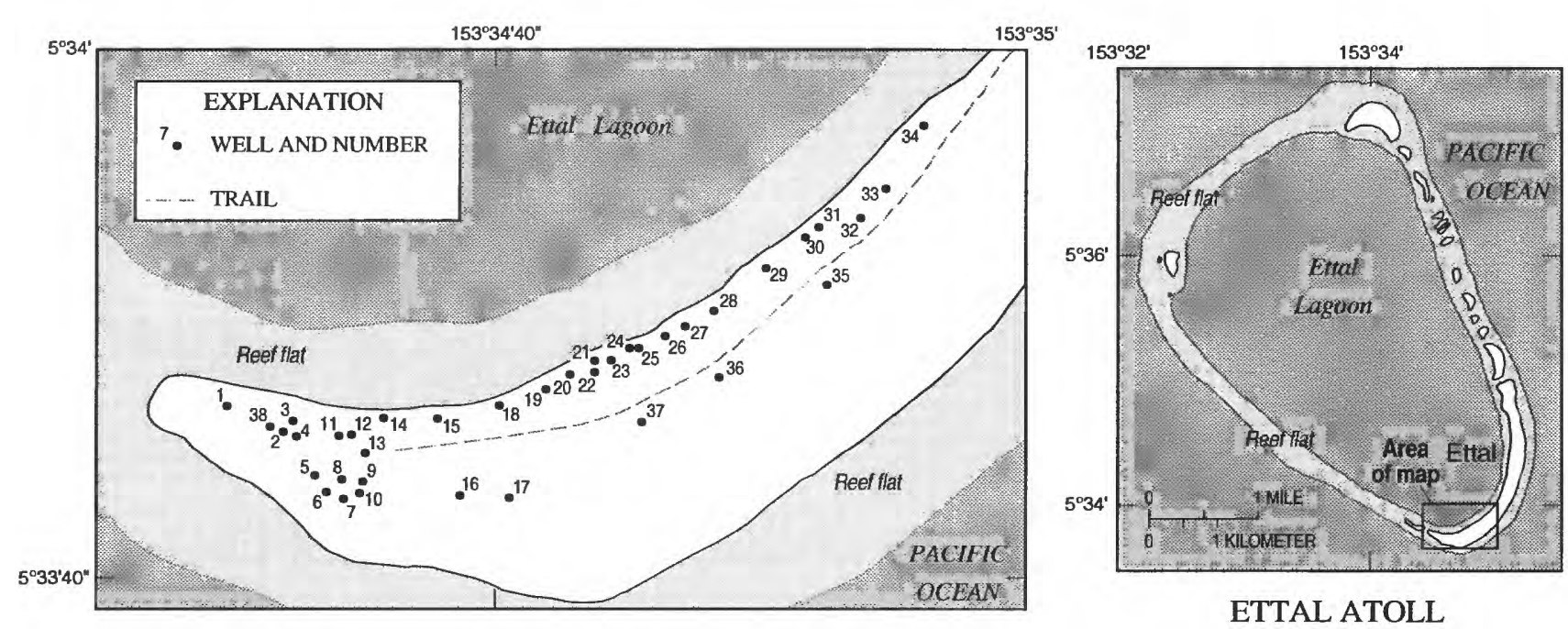

ETTAL ATOLL
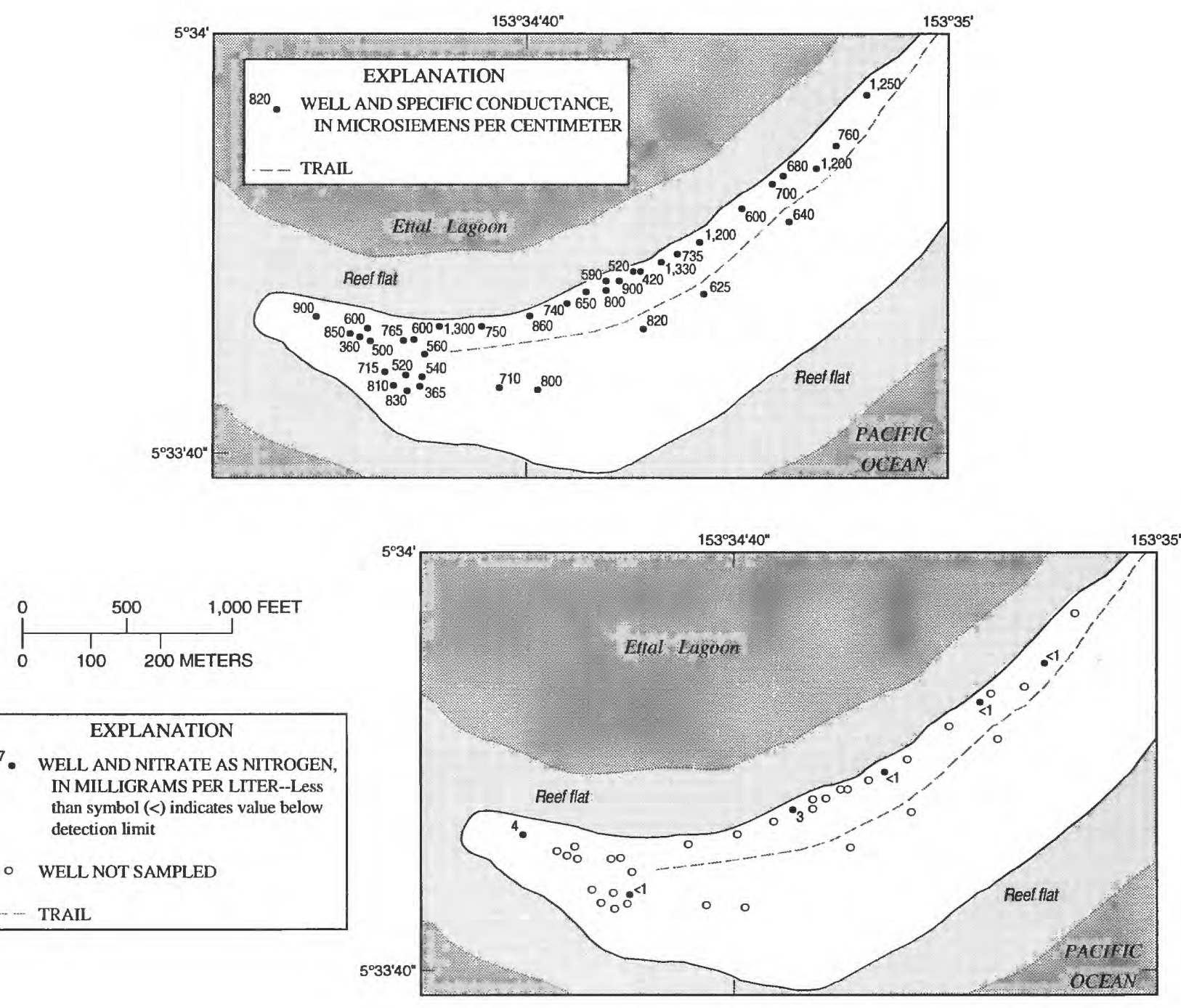

Figure 30. Well locations, specific conductance, and nitrate concentrations for Ettal Island, Ettal Atoll, Mortlock Island Group, Chuuk State, 1984. 


\section{Lukunoch Island}

The population of Lukunoch Island at Lukunoch Atoll is about 668 (Truk State Office of Planning and Statistics, 1981). Well locations, specific conductance, and nitrate concentrations for Lukunoch Island are shown in figure 31 . Selected physical and water-quality data for the 56 dug wells sampled are given in table 23 . The mean specific conductance for water from 56 wells was $830 \mu \mathrm{S} / \mathrm{cm}$ and ranged from 400 to $2,600 \mu \mathrm{S} / \mathrm{cm}$. On the basis of the estimated chloride concentrations from specific conductance data, 1 of the 56 wells sampled (well 9) exceeded the WHO (1984) guideline for chloride. Thirty-three of the 56 wells sampled were field tested for nitrate concentration. Water from all of the wells tested had nitrate concentrations of less than 1 $\mathrm{mg} / \mathrm{L}$ (as $\mathrm{N}$ ), except one, which had a concentration of $4 \mathrm{mg} / \mathrm{L}$ (as N).

Table 23. Selected physical and water-quality data from dug wells, October 25, 1984, Lukunoch Island, Lukunoch Atoll, Mortlock Island Group

$\left[{ }^{\circ} \mathrm{C}\right.$, degrees Celsius; $\mu \mathrm{S} / \mathrm{cm}$, microsiemens per centimeter at $25^{\circ} \mathrm{C} ; \mathrm{Cl}$, chloride; $\mathrm{NO}_{3}$, nitrate; $\mathrm{mg} / \mathrm{L}$, milligrams per liter; --, not measured; <, less than]

\begin{tabular}{|c|c|c|c|c|c|c|c|}
\hline $\begin{array}{l}\text { Well } \\
\text { no. }\end{array}$ & Owner & $\begin{array}{c}\text { Temperature } \\
\left({ }^{\circ} \mathbf{C}\right)\end{array}$ & $\begin{array}{c}\text { Specific } \\
\text { conductance } \\
(\mu \mathrm{S} / \mathrm{cm})\end{array}$ & pH & $\begin{array}{c}\mathrm{Cl} \\
(\mathrm{mg} / \mathrm{L})\end{array}$ & $\begin{array}{c}\mathrm{NO}_{3,} \\
\text { as } \mathrm{N} \\
\text { (mg/l) }\end{array}$ & Remarks \\
\hline 1 & Mwarluk & 27 & 1,000 & - & - & $<1$ & \\
\hline 2 & Linn & 28 & 1,100 & -- & - & $<1$ & \\
\hline 3 & Puas & 28 & 475 & -- & - & $<1$ & \\
\hline 4 & Murito & 27 & 850 & - & - & $<1$ & \\
\hline 5 & Tasi & 28 & 750 & - & - & $<1$ & \\
\hline 6 & Mispi & 29 & 690 & - & - & $<1$ & \\
\hline 7 & Rayphano & 28 & 680 & -- & - & $<1$ & \\
\hline 8 & Robert & 28 & 540 & - & - & $<1$ & \\
\hline 9 & not known & -- & 2,600 & -- & - & -- & \\
\hline 10 & Hainrick & 28.5 & 790 & -- & - & $<1$ & \\
\hline 11 & Sitro & 28 & 560 & -- & - & $<1$ & \\
\hline 12 & Puas & 29.5 & 680 & -- & 18 & $<1$ & \\
\hline 13 & Rafael & 29 & 920 & -- & - & $<1$ & \\
\hline 14 & Robert & 29.5 & 1,300 & -- & - & 4 & \\
\hline 15 & Sido & 27 & 520 & -- & - & $<1$ & \\
\hline 16 & Nennis & 28.5 & 650 & - & - & $<1$ & \\
\hline 17 & Luke & 28 & 520 & - & - & $<1$ & \\
\hline 18 & Malia & 27 & 1,200 & - & - & $<1$ & \\
\hline 19 & Tefant & 27 & 810 & - & - & $<1$ & \\
\hline 20 & Fichipwe & 26 & 1,000 & -- & - & $<1$ & \\
\hline 21 & Simon & 28 & 700 & -- & - & $<1$ & \\
\hline 22 & Wenio & 28 & 680 & -- & - & $<1$ & \\
\hline 23 & Elgin & 27 & 725 & -- & - & $<1$ & \\
\hline 24 & Elginbwi & 29 & 1,180 & - & - & $<1$ & \\
\hline 25 & Marluk & 27.5 & 875 & - & - & $<1$ & \\
\hline 26 & Sekarb & 27 & 700 & - & -- & $<1$ & \\
\hline 27 & Ikelap & 28 & 825 & -- & - & $<1$ & \\
\hline 28 & Samson & 27.5 & 840 & - & - & $<1$ & \\
\hline 29 & Rayphan & 28 & 760 & -- & - & $<1$ & \\
\hline 30 & Malia & 28 & 540 & -- & - & $<1$ & \\
\hline 31 & Samson & 28 & 790 & -- & - & $<1$ & \\
\hline
\end{tabular}


Table 23. Selected physical and water-quality data from dug wells, October 25, 1984, Lukunoch Island, Lukunoch Atoll, Mortlock Island Group--Continued

\begin{tabular}{|c|c|c|c|c|c|c|c|}
\hline $\begin{array}{l}\text { Well } \\
\text { no. }\end{array}$ & Owner & $\begin{array}{c}\text { Temperature } \\
\text { (ㄷ) }\end{array}$ & $\begin{array}{c}\text { Specific } \\
\text { conductance } \\
(\mu \mathrm{S} / \mathrm{cm})\end{array}$ & pH & $\underset{\text { (mg/L) }}{\mathrm{Cl}}$ & $\begin{array}{c}\mathrm{NO}_{3}, \\
\text { as N } \\
\text { (mg/L) }\end{array}$ & Remarks \\
\hline 32 & Sekarb & 29 & 820 & - & - & - & \\
\hline 33 & Setir & 27 & 640 & -- & -- & -- & \\
\hline 34 & Katril & 27 & 450 & - & -- & -- & \\
\hline 35 & Elginpwi & 27.5 & 900 & 7.0 & 73 & $<1$ & Major ion and trace element sample \\
\hline 36 & Eparisto & 27 & 1,150 & - & - & -- & \\
\hline 37 & Rabuda & 28 & 675 & -- & -- & $<1$ & \\
\hline 38 & Kiyoshi & 27 & 760 & - & -- & - & \\
\hline 39 & Likichimus & 27 & 1,150 & - & - & -- & \\
\hline 40 & Pulaua & 28 & 690 & -- & -- & -- & \\
\hline 41 & Chol & 28 & 780 & -- & -- & -- & \\
\hline 42 & Wenio & 28 & 1,200 & -- & -- & - & \\
\hline 43 & Isasio & 27.5 & 675 & -- & - & -- & \\
\hline 44 & Kamilo & 28 & 1,000 & -- & -- & - & \\
\hline 45 & Eseteb & 27 & 1,000 & - & 73 & - & \\
\hline 46 & Likishe & 27.5 & 750 & -- & -- & $<1$ & \\
\hline 47 & Puas & 28.5 & 800 & -- & - & - & \\
\hline 48 & Puas & 28 & 800 & - & - & - & \\
\hline 49 & Thomas & 27 & 800 & - & -- & - & \\
\hline 50 & Kosy & 27.5 & 1,000 & - & -- & - & \\
\hline 51 & Patricio & 27 & 1,200 & - & -- & - & \\
\hline 52 & Nick & 28 & 700 & - & -- & - & \\
\hline 53 & Apong & 27 & 650 & - & - & -- & \\
\hline 54 & Marluk & 27 & 750 & - & - & - & \\
\hline 55 & Pilas & 27.5 & 400 & -- & -- & - & \\
\hline 56 & Sebastian & 27.5 & 525 & - & -- & - & \\
\hline
\end{tabular}



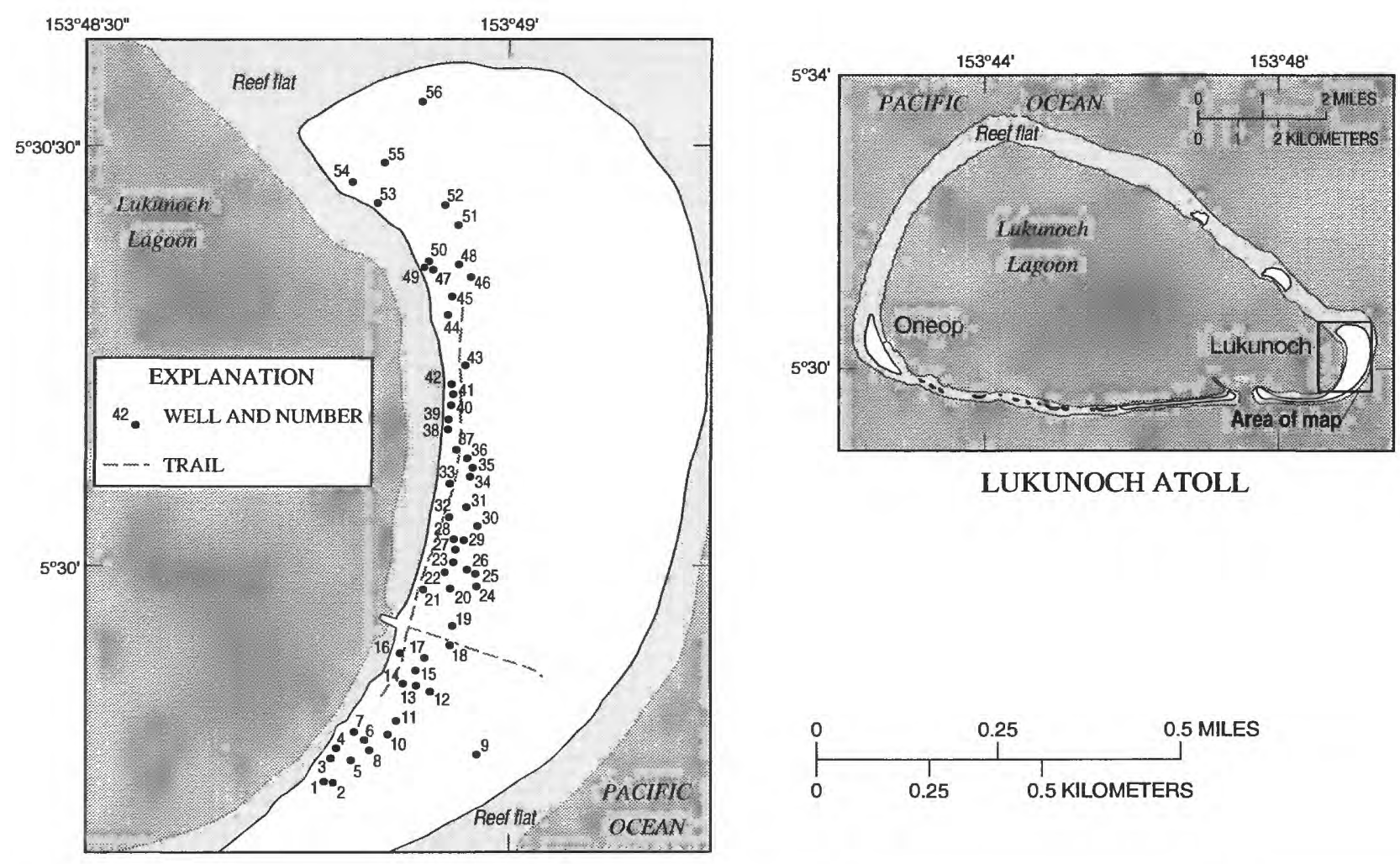

LUKUNOCH ATOLL
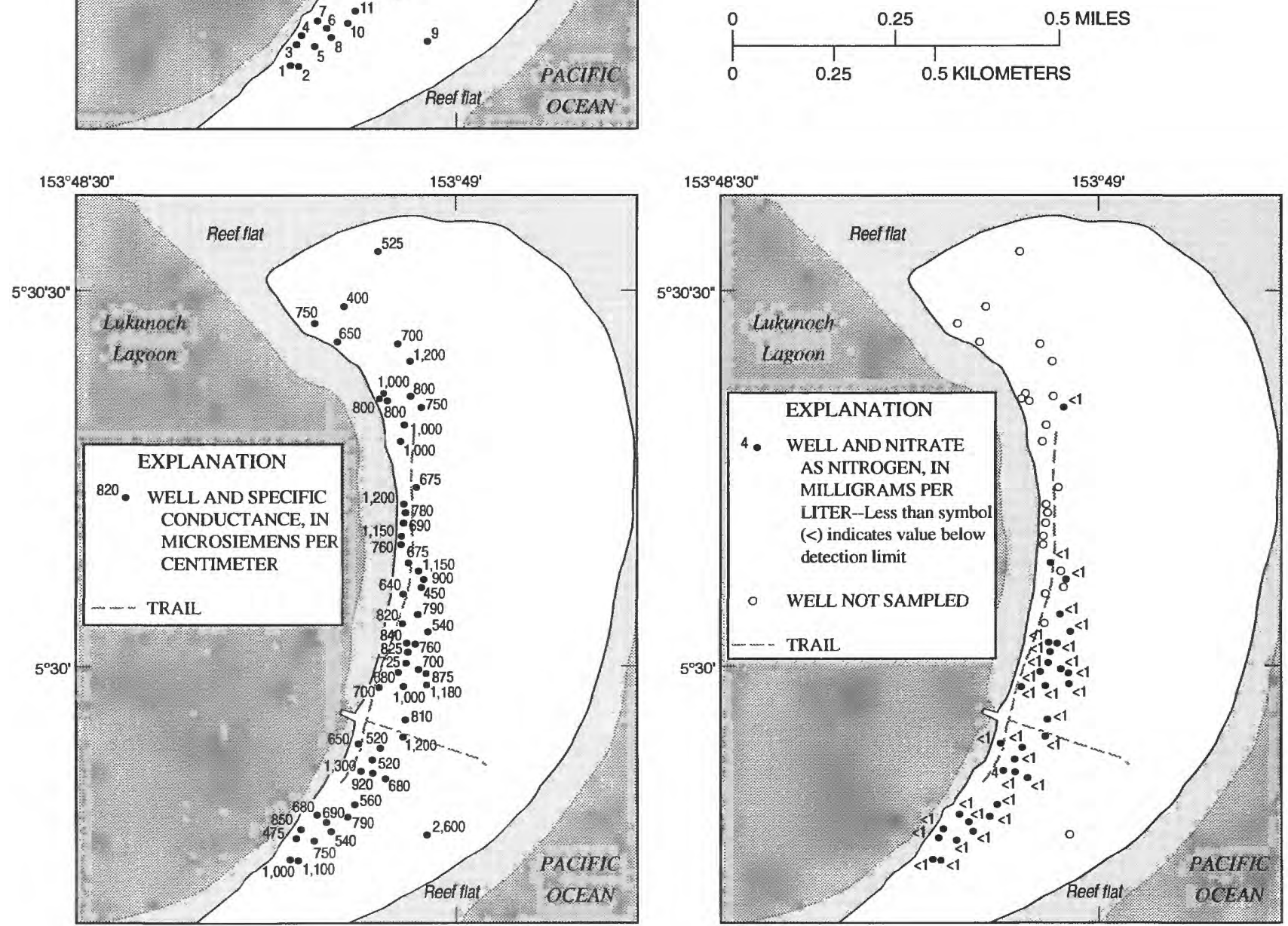

Figure 31. Well locations, specific conductance, and nitrate concentrations for Lukunoch Island, Lukunoch Atoll, Mortlock Island Group, Chuuk State, 1985. 


\section{Satowan Island}

The population of Satowan Island at Satowan Atoll is about 766 (Truk State Office of Planning and Statistics, 1981). Well locations, specific conductance, and nitrate concentrations for Satowan Island are shown in figure 32. Selected physical and water-quality data for the 63 dug wells and 1 driven well sampled are given in table 24. The mean specific conductance for water from
64 wells was $710 \mu \mathrm{S} / \mathrm{cm}$ and ranged from 210 to 1,700 $\mu \mathrm{S} / \mathrm{cm}$. On the basis of the estimated chloride concentrations from specific conductance data, water from one well (well 57) exceeded the WHO (1984) guideline for chloride. Thirty-eight of the 61 wells sampled were field tested for nitrate concentration. Water from 32 of the 38 wells tested had nitrate concentrations of less than $1 \mathrm{mg} / \mathrm{L}$ (as $\mathrm{N}$ ); the remaining samples had concentrations ranging from 1 to $5.5 \mathrm{mg} / \mathrm{L}$ (as $\mathrm{N}$ ).

Table 24. Selected physical and water-quality data from dug wells, October 22, 23, and 27, 1984, Satowan Island, Satowan Atoll, Mortlock Island Group

$\left[{ }^{\circ} \mathrm{C}\right.$, degrees Celsius; $\mu \mathrm{S} / \mathrm{cm}$, microsiemens per centimeter at $25^{\circ} \mathrm{C} ; \mathrm{Cl}$, chloride; $\mathrm{NO}_{3}$, nitrate; $\mathrm{mg} / \mathrm{L}$, milligrams per liter; --, not measured; <, less than]

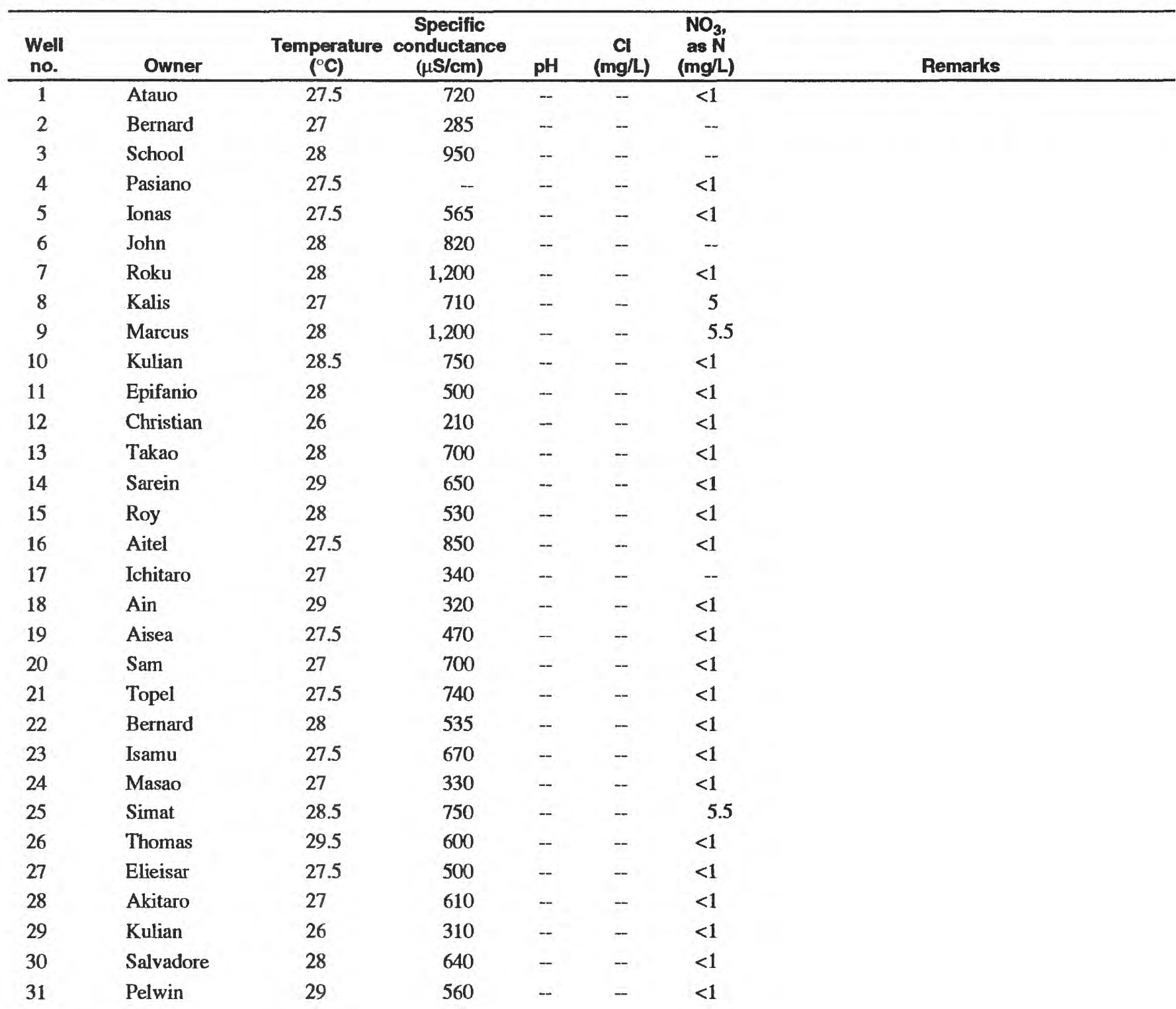


Table 24. Selected physical and water-quality data from dug wells, October 22, 23, and 27, 1984, Satowan Island, Satowan Atoll, Mortlock Island Group--Continued

\begin{tabular}{|c|c|c|c|c|c|c|c|}
\hline $\begin{array}{l}\text { Well } \\
\text { no. }\end{array}$ & Owner & $\begin{array}{c}\text { Temperature } \\
\left({ }^{\circ} \mathrm{C}\right)\end{array}$ & $\begin{array}{c}\text { Specific } \\
\text { conductance } \\
(\mu \mathrm{S} / \mathrm{cm})\end{array}$ & pH & $\begin{array}{c}\mathrm{Cl} \\
\text { (mg/L) }\end{array}$ & $\begin{array}{l}\mathrm{NO}_{3} \\
\text { as N } \\
\text { (mg/L) }\end{array}$ & Remarks \\
\hline 32 & Titus & 28 & 840 & - & - & $<1$ & \\
\hline 33 & Siro & 28 & 1,150 & -- & -- & $<1$ & \\
\hline 34 & Raymond & 28 & 690 & -- & -- & $<1$ & \\
\hline 35 & Lewis & 29.5 & 430 & -- & -- & -- & \\
\hline 36 & Simeon & 28 & 300 & -- & -- & $<1$ & \\
\hline 37 & Tasiro & 30 & 440 & -- & -- & $<1$ & \\
\hline 38 & Misauo & 27.5 & 395 & 7.1 & 11 & 1 & $\begin{array}{l}\text { Installed } 11 / 4 \text { " well point with hand pump. Major } \\
\text { ion and trace element sample }\end{array}$ \\
\hline 39 & Santiago & 28 & 440 & -- & -- & -- & \\
\hline 40 & Sian & 26.5 & 650 & - & - & -- & \\
\hline 41 & Antonio & 27 & 540 & -- & - & -- & \\
\hline 42 & Steven & 28 & 900 & - & - & -- & \\
\hline 43 & Thomas & 26.5 & 725 & - & -- & -- & \\
\hline 44 & Tamian & 28 & 800 & - & -- & -- & \\
\hline 45 & MataichiL & 28 & 850 & -- & -- & -- & \\
\hline 46 & Noel & 27 & 900 & -- & -- & -- & \\
\hline 47 & Kilian & 27 & 540 & -- & -- & -- & \\
\hline 48 & Akostian & 28 & 950 & -- & - & -- & \\
\hline 49 & Haritos & 27 & 850 & -- & - & -- & . \\
\hline 50 & Florian & 26 & 495 & -- & -- & -- & \\
\hline 51 & Moufa & 27 & 850 & -- & - & -- & \\
\hline 52 & Isau & 27 & 1,000 & -- & - & -- & \\
\hline 53 & Filomen & 27.5 & 950 & -- & -- & -- & \\
\hline 54 & Penjamin & 28.5 & 1,000 & -- & -- & -- & \\
\hline 55 & Joseph & 27 & 840 & -- & - & - & \\
\hline 56 & Aleper & 27 & 1,100 & -- & -- & -- & \\
\hline 57 & Amon & 27 & 1,700 & -- & -- & -- & \\
\hline 58 & Simon & 27 & 340 & - & - & -- & \\
\hline 59 & Tas & 27.5 & 1,100 & -- & -- & -- & \\
\hline 60 & Girl's dorm & 29 & 1,150 & - & - & 22 & \\
\hline 61 & Boy's dorm & 27.5 & 695 & -- & - & 22 & \\
\hline A1 & Misauo & 26.5 & 280 & -- & - & $<1$ & \\
\hline A43 & Jack & 27 & 580 & -- & -- & $<1$ & \\
\hline A44 & Kilaimer & 26.5 & 438 & -- & -- & $<1$ & \\
\hline
\end{tabular}



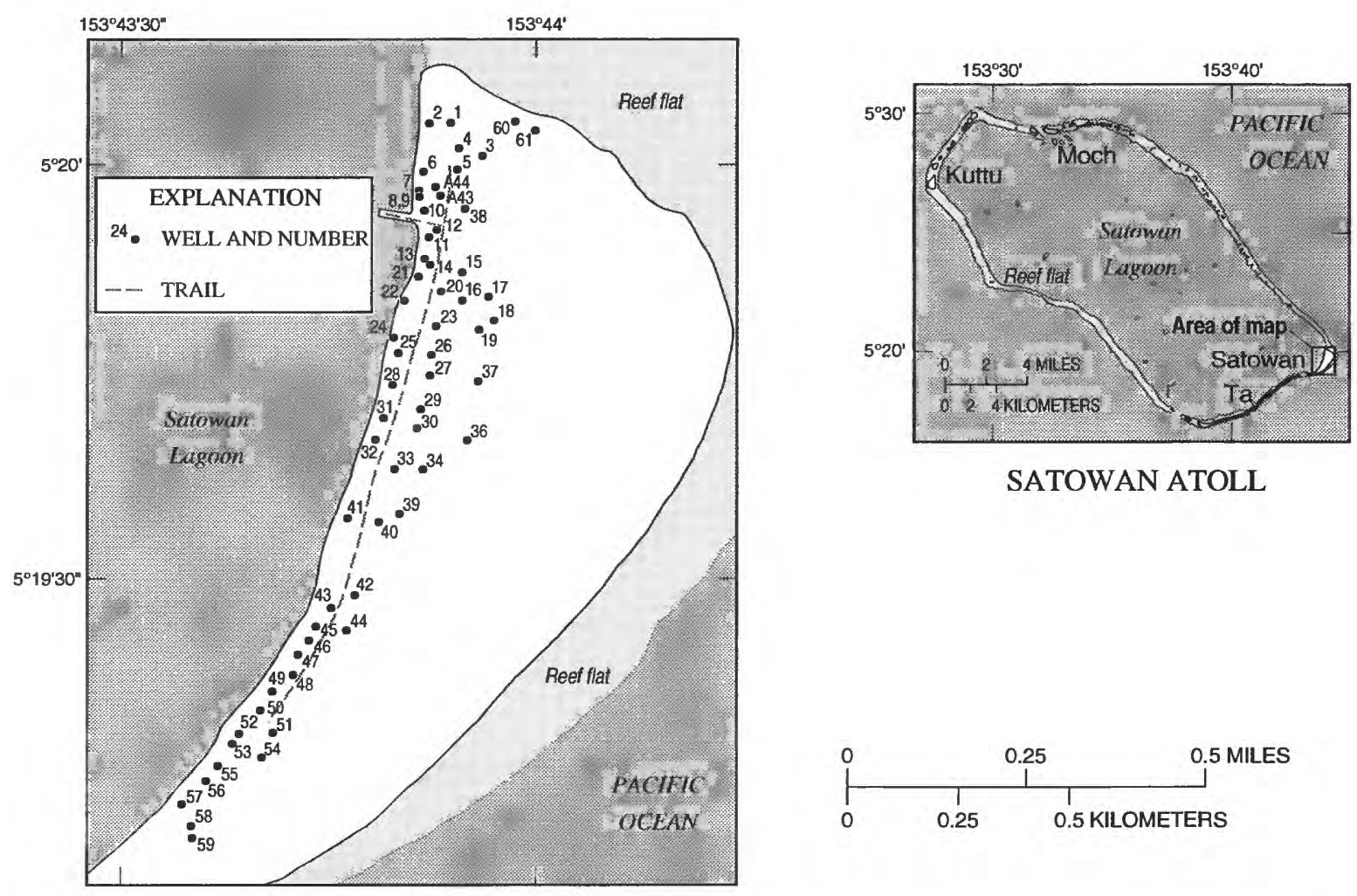

\section{SATOWAN ATOLL}
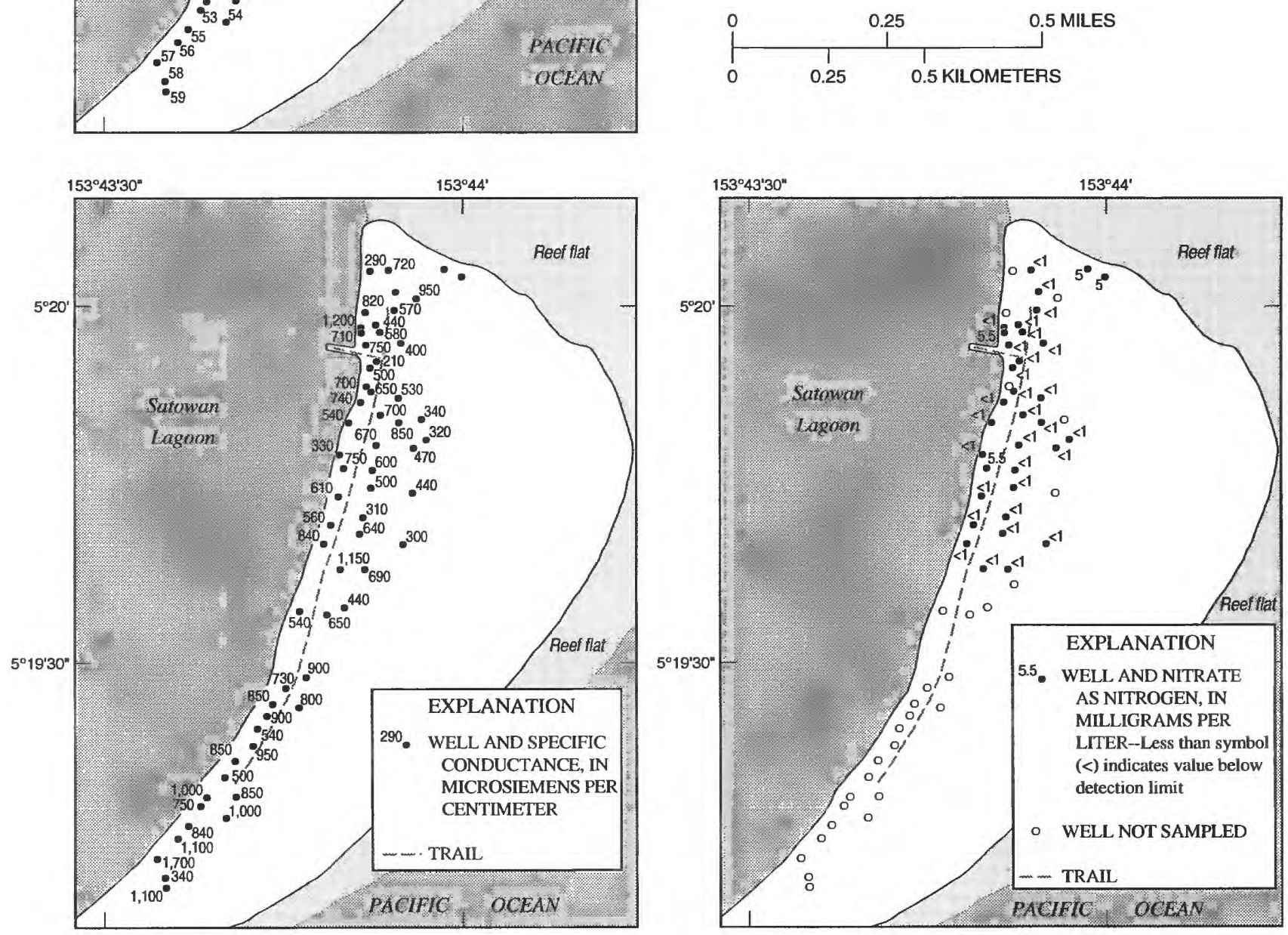

Figure 32. Well locations, specific conductance, and nitrate concentrations for Satowan Island, Satowan Atoll, Mortlock Island Group, Chuuk State, 1985. 


\section{Oneop Island}

The population of Oneop Island at Lukunoch Atoll is about 485 (Truk State Office of Planning and Statistics, 1981). Well locations, specific conductance, and nitrate concentrations for Oneop Island are shown in figure 33. Selected physical and water-quality data for the 29 dug wells and 1 driven well sampled are given in table 25 . The mean specific conductance for water from
30 wells was $850 \mu \mathrm{S} / \mathrm{cm}$ and ranged from 330 to 1,500 $\mu \mathrm{S} / \mathrm{cm}$. On the basis of the estimated chloride concentrations from specific conductance data, none of the water samples exceeded the WHO (1984) guideline for chloride. Twenty-six of the 30 wells sampled were field tested for nitrate concentration. Water from 21 of the 26 wells tested had nitrate concentrations of less than 1 $\mathrm{mg} / \mathrm{L}$ (as $\mathrm{N}$ ); the remaining samples had concentrations ranging from 4 to $10 \mathrm{mg} / \mathrm{L}$ (as $\mathrm{N}$ ).

Table 25. Selected physical and water-quality data from dug wells, February 3, 1984, Oneop Island, Lukunoch Atoll, Mortlock Island Group

$\left[{ }^{\circ} \mathrm{C}\right.$, degrees Celsius; $\mu \mathrm{S} / \mathrm{cm}$, microsiemens per centimeter at $25^{\circ} \mathrm{C} ; \mathrm{Cl}$, chloride; $\mathrm{NO}_{3}$, nitrate; $\mathrm{mg} / \mathrm{L}$, milligrams per liter, --, not measured; <, less than]

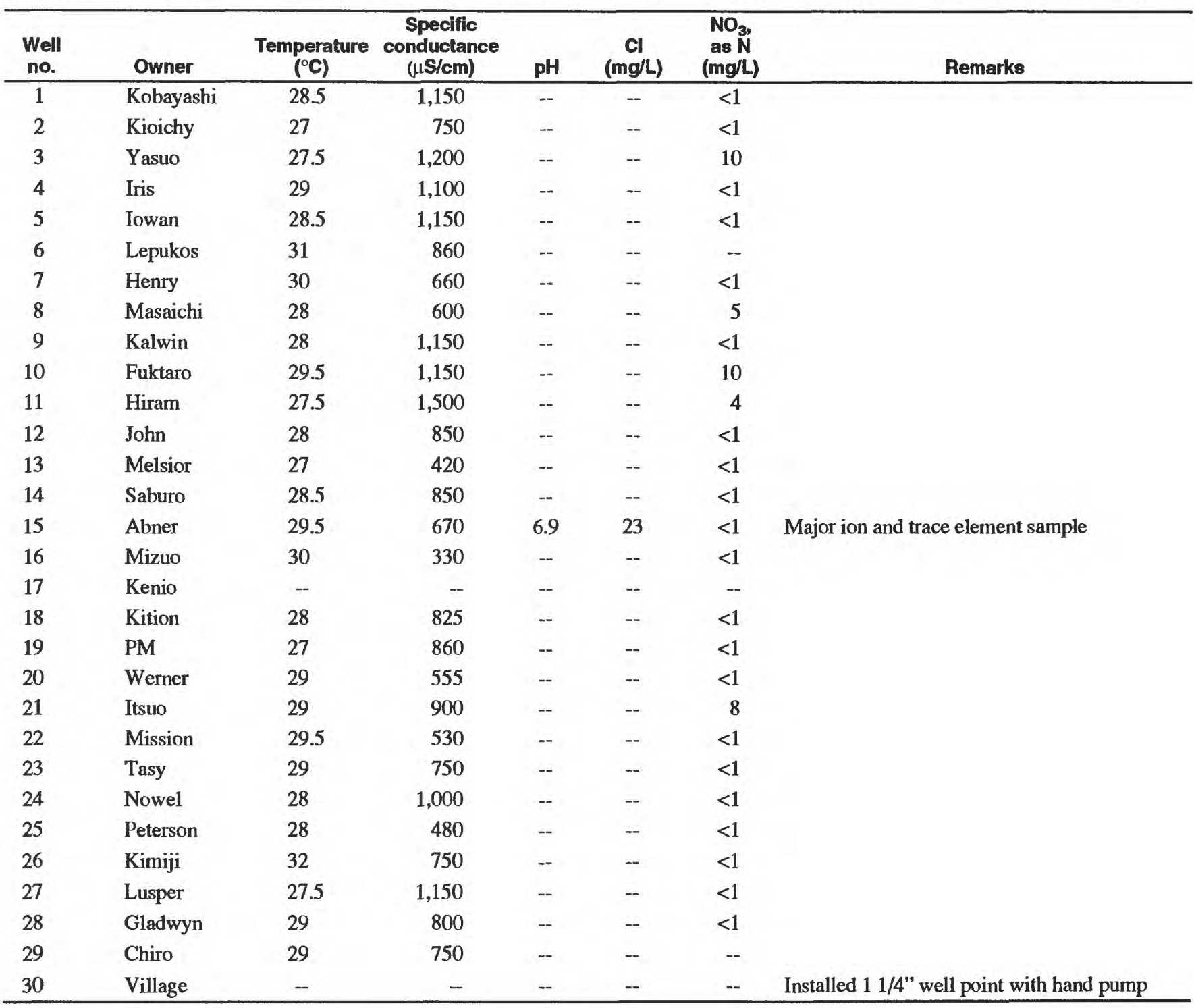



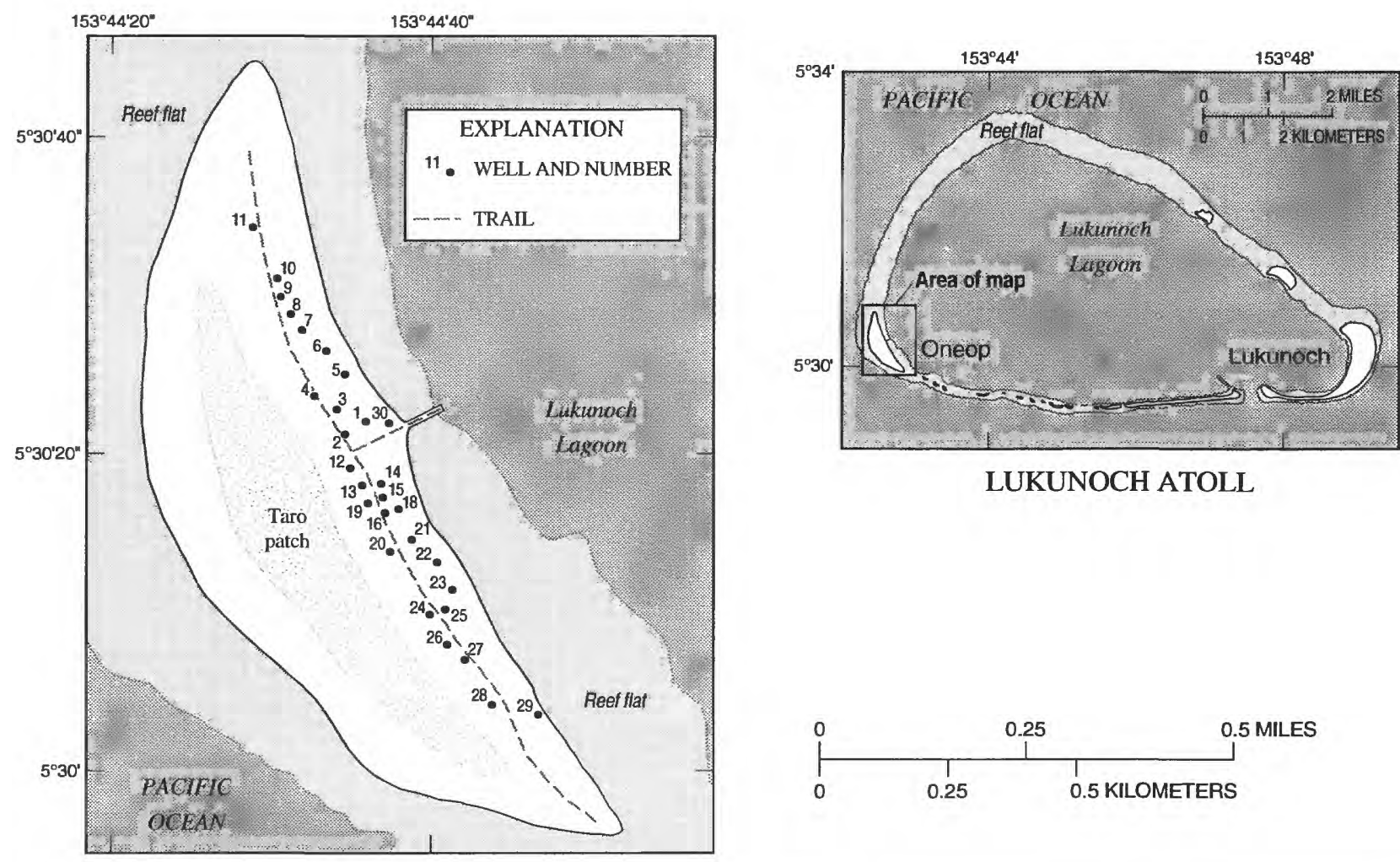

LUKUNOCH ATOLL
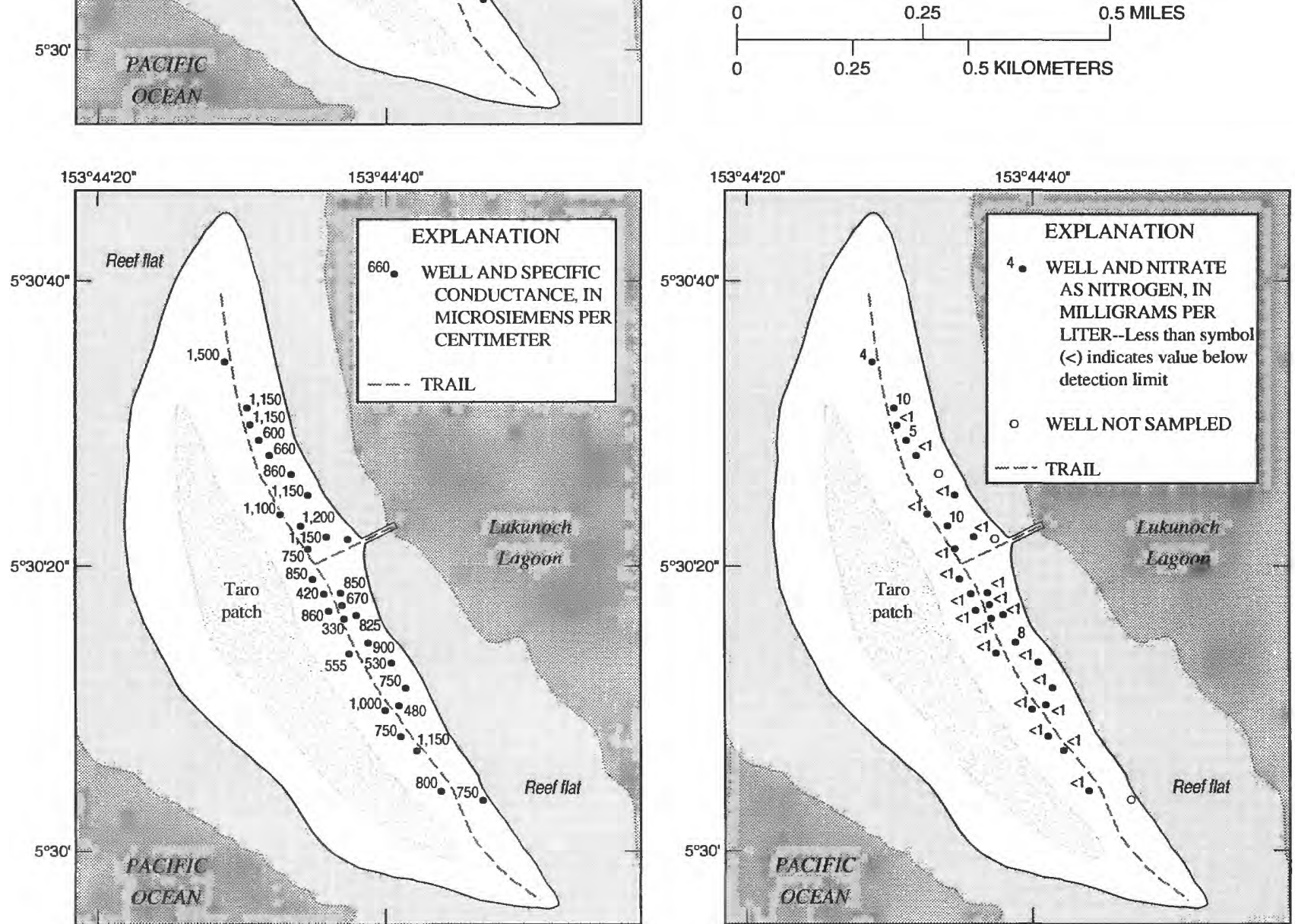

Figure 33. Well locations, specific conductance, and nitrate concentrations for Oneop Island, Lukunoch Atoll, Mortlock Island Group, Chuuk State, 1985. 


\section{Ta Island}

The population of Ta Island at Satowan Atoll is about 294 (Truk State Office of Planning and Statistics, 1981). Well locations, specific conductance, and nitrate concentrations for Ta Island are shown in figure 34.

Selected physical and water-quality data for the $17 \mathrm{dug}$ wells, 1 driven well, and 1 taro patch site sampled are given in table 26. The mean specific conductance for water from 18 wells was $850 \mu \mathrm{S} / \mathrm{cm}$ and ranged from 180 to $2,000 \mu \mathrm{S} / \mathrm{cm}$. On the basis of the estimated chloride concentrations from specific conductance data, water from two wells (wells 14 and 15) exceeded the WHO (1984) guideline for chloride. One of the 18 wells sampled was field tested for nitrate concentration, which were less than $1 \mathrm{mg} / \mathrm{L}$ (as N).

Table 26. Selected physical and water-quality data from dug and driven wells, and taro patch site, October 26-27, 1985, Ta Island, Satowan Atoll, Mortlock Island Group

$\left[{ }^{\circ} \mathrm{C}\right.$, degrees Celsius; $\mu \mathrm{S} / \mathrm{cm}$, microsiemens per centimeter at $25^{\circ} \mathrm{C} ; \mathrm{Cl}$, chloride; $\mathrm{NO}_{3}$, nitrate; $\mathrm{mg} / \mathrm{L}$, milligrams per liter; --, not measured; <, less than]

\begin{tabular}{|c|c|c|c|c|c|c|c|}
\hline $\begin{array}{l}\text { Well } \\
\text { no. }\end{array}$ & Owner & $\begin{array}{c}\text { Temperature } \\
\left.\text { ( }{ }^{\circ} \mathbf{C}\right)\end{array}$ & $\begin{array}{c}\text { Specific } \\
\text { conductance } \\
(\mu \mathrm{S} / \mathrm{cm})\end{array}$ & pH & $\begin{array}{c}\mathrm{Cl} \\
(\mathrm{mg} / \mathrm{L})\end{array}$ & $\begin{array}{c}\mathrm{NO}_{3}, \\
\text { as N } \\
\text { (mg/L) }\end{array}$ & Remarks \\
\hline 1 & Siales & 28 & 950 & -- & -- & - & \\
\hline 3 & Eimil & 28 & 1,320 & - & -- & -- & \\
\hline 4 & Eifram & 29 & 750 & - & - & -- & \\
\hline 7 & Kulio & 27 & 385 & - & -- & - & \\
\hline 8 & Sakios & 27 & 180 & - & -- & - & \\
\hline 9 & Akisima & 29 & 975 & - & - & -- & \\
\hline 10 & Herwin & 27 & 1,080 & -- & -- & -- & \\
\hline 15 & Eli & 27 & 1,590 & -- & - & -- & \\
\hline 16 & Suap & 27 & 700 & -- & -- & -- & \\
\hline 17 & Achu & 27 & 800 & - & -- & -- & \\
\hline 18 & Village & 27.5 & 860 & 7.1 & 100 & $<1$ & Installed $11 / 4$ " well point with hand pump \\
\hline Taro patch & & -- & 280 & -- & -- & - & \\
\hline
\end{tabular}



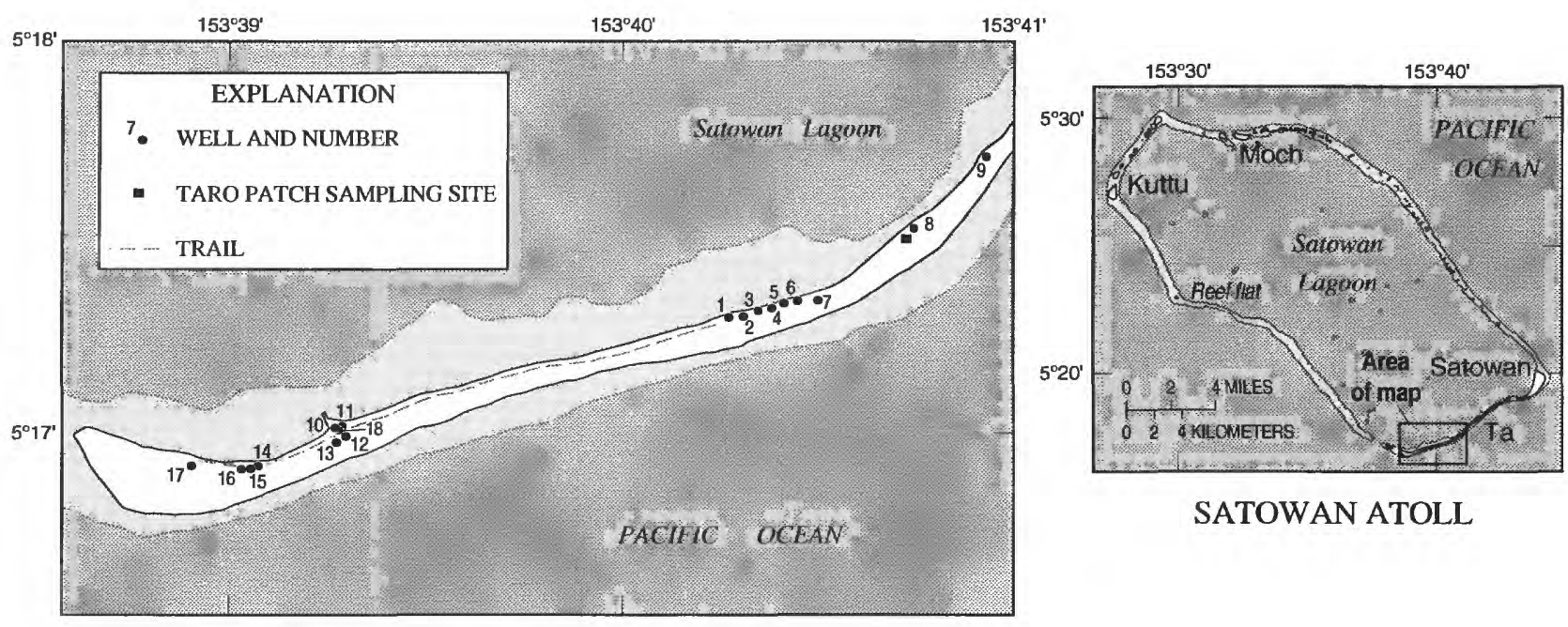

SATOWAN ATOLL
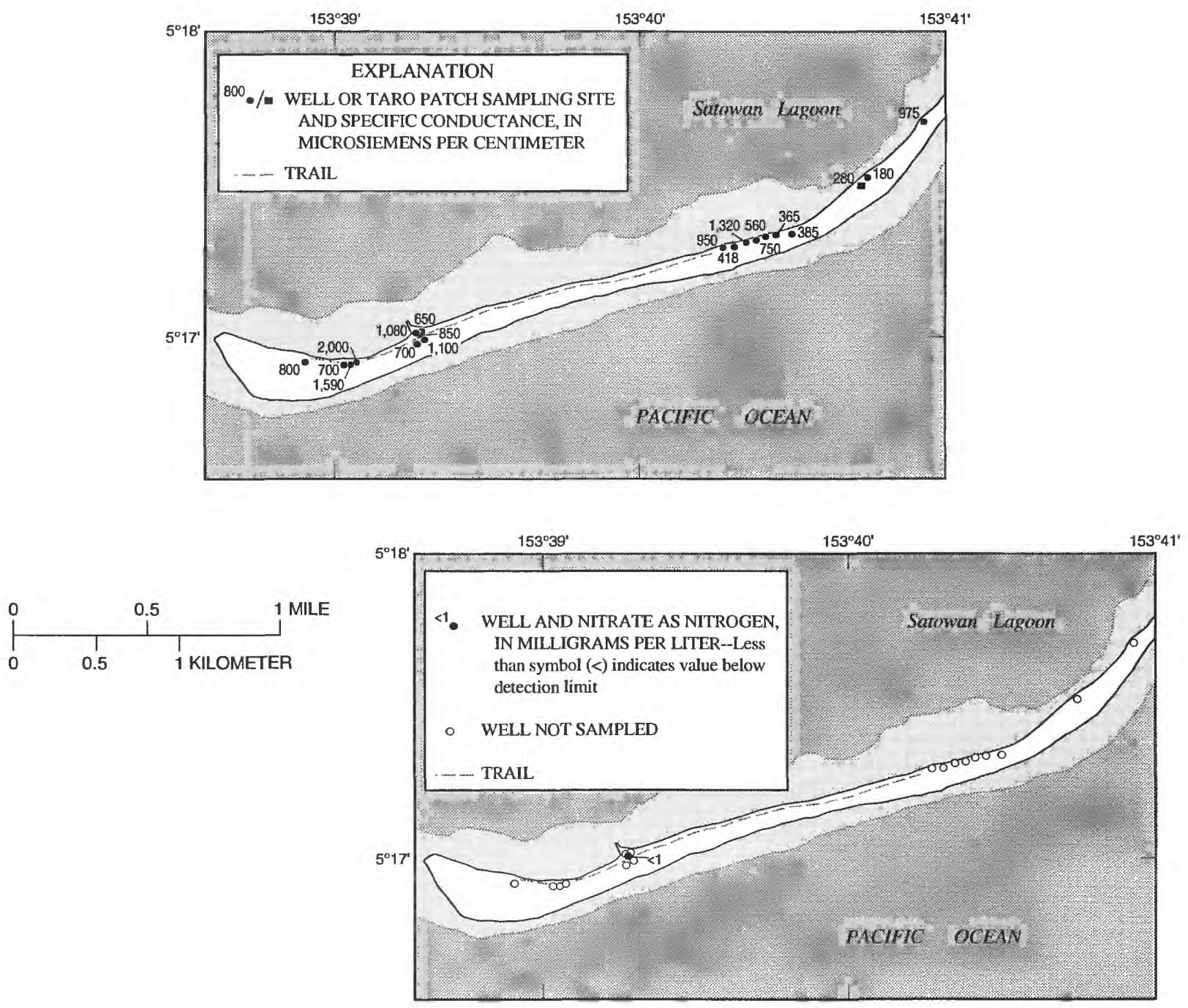

Figure 34. Well locations, specific conductance, and nitrate concentrations for Ta Island, Satowan Atoll, Mortlock Island Group, Chuuk State, 1984. 


\section{Kuttu Island}

The population of Kuttu Island at Satowan Atoll is about 484 (Truk State Office of Planning and Statistics, 1981). Well locations, sampling sites, specific conductance, and nitrate concentrations for Kuttu Island are shown in figure 35 . Selected physical and water-quality data for the 30 dug wells and 8 taro patch sites sampled are given in table 27 . The mean specific conductance for water from 30 wells was $1,090 \mu \mathrm{S} / \mathrm{cm}$ and ranged from 550 to $6,000 \mu \mathrm{S} / \mathrm{cm}$. On the basis of the estimated chloride concentrations from specific conductance data, water from 2 of the 30 wells sampled (wells 6 and 13) exceeded the WHO (1984) guideline for chloride. Ten of the 30 wells sampled were field tested for nitrate concentration. Water from 7 of the 10 wells tested had nitrate concentrations of less than $1 \mathrm{mg} / \mathrm{L}$ (as $\mathrm{N}$ ); the remaining samples had concentrations ranging from 7 to $14 \mathrm{mg} / \mathrm{L}$ (as N). One of these samples (well 7) exceeded the WHO (1984) guideline for nitrate.

Table 27. Selected physical and water-quality data from dug wells and taro patch sites, February 17, 1984, Kuttu Island, Satowan Atoll, Mortlock Island Group

$\left[{ }^{\circ} \mathrm{C}\right.$, degrees Celsius; $\mu \mathrm{S} / \mathrm{cm}$, microsiemens per centimeter at $25^{\circ} \mathrm{C} ; \mathrm{Cl}$, chloride; $\mathrm{NO}_{3}$, nitrate; $\mathrm{mg} / \mathrm{L}$, milligrams per liter; --, not measured; <, less than; $>$, greater than]

\begin{tabular}{|c|c|c|c|c|c|c|c|}
\hline Well no. & Owner & $\begin{array}{c}\text { Temperature } \\
\left({ }^{\circ} \mathrm{C}\right)\end{array}$ & $\begin{array}{c}\text { Specific } \\
\text { conductance } \\
(\mu \mathrm{S} / \mathrm{cm})\end{array}$ & pH & $\begin{array}{c}\mathrm{Cl} \\
(\mathrm{mg} / \mathrm{L})\end{array}$ & $\begin{array}{c}\mathrm{NO}_{3} \\
\text { as } \mathrm{N} \\
(\mathrm{mg} / \mathrm{L})\end{array}$ & Remarks \\
\hline 1 & Luela & 27 & 1,050 & 6.8 & - & -- & \\
\hline 2 & Diofil & 26.5 & 975 & 6.9 & -- & -- & \\
\hline 3 & Kapino & 26.5 & 1,000 & 7.0 & -- & -- & \\
\hline 4 & Kos & 26.5 & 925 & 7.0 & -- & $<1$ & \\
\hline 5 & Masuo & 26 & 1,020 & 7.3 & -- & -- & \\
\hline 6 & Elias & 26.5 & 1,700 & 7.0 & -- & -- & \\
\hline 7 & Daniel & 27.5 & 1,280 & 7.0 & -- & 14 & \\
\hline 8 & Roman & 27 & 850 & 7.2 & - & 7 & \\
\hline 9 & Ernest & 27 & 1,100 & 7.0 & -- & $<1$ & \\
\hline 10 & Hirr & 26.5 & 625 & 7.1 & -- & $<1$ & \\
\hline 11 & Rufino & 26.5 & 550 & 7.4 & -- & $<1$ & \\
\hline 12 & Akostin & 26.5 & 740 & 7.3 & -- & -- & \\
\hline 13 & Istino & 26 & 6,000 & 7.3 & 1,600 & - & \\
\hline 14 & Eriper & 26.5 & 725 & 7.3 & -- & -- & \\
\hline 15 & Tasiuo & 27 & 1,150 & 7.1 & 23 & -- & \\
\hline 16 & Atalifus & 26.5 & 600 & 7.3 & - & -- & \\
\hline 17 & Akira & 26 & 800 & 7.2 & -- & $<1$ & \\
\hline 18 & Sami & 27 & 1,120 & 6.9 & -- & -- & \\
\hline 19 & Daifus & 26.5 & 875 & 7.2 & -- & -- & \\
\hline 20 & Damel & 27 & 650 & 7.4 & -- & $<1$ & \\
\hline 21 & Tarech & 26.5 & 700 & 7.1 & 8 & -- & \\
\hline 22 & Epel & 26.5 & 750 & 7.1 & -- & - & \\
\hline 23 & Berman & 26.5 & 880 & 7.0 & - & -- & \\
\hline 24 & Dofer & 26.5 & 1,320 & 7.0 & -- & -- & \\
\hline 25 & Sam & 26.5 & 1,150 & 6.9 & - & -- & \\
\hline 26 & Achon & 26 & 710 & 7.4 & 15 & -- & \\
\hline 27 & Asauo & 26.5 & 1,200 & 6.9 & - & - & \\
\hline 28 & Kutper & 27 & 750 & 7.4 & 45 & 8 & Major ion and trace element sample \\
\hline 29 & Santer & 26.5 & 825 & 7.1 & -- & $<1$ & \\
\hline 30 & Michael & 26 & 625 & 7.2 & - & -- & \\
\hline \multicolumn{2}{|c|}{ Taro patch 1} & 27 & 4,500 & - & -- & -- & \\
\hline \multicolumn{2}{|c|}{ Taro patch 2} & 27 & 4,500 & - & - & -- & \\
\hline \multicolumn{2}{|c|}{ Taro patch 3} & 28 & 3,500 & -- & - & -- & \\
\hline \multicolumn{2}{|c|}{ Taro patch 4} & 28 & 255 & 7.7 & -- & - & \\
\hline \multicolumn{2}{|c|}{ Taro patch 5} & 27.5 & 155 & 7.2 & -- & -- & \\
\hline \multicolumn{2}{|c|}{ Taro patch 6} & 28.5 & $>8000$ & - & -- & - & \\
\hline \multicolumn{2}{|c|}{ Taro patch $6 \mathrm{~A}$} & 28 & 3,500 & -- & - & -- & \\
\hline \multicolumn{2}{|c|}{ Taro patch 7A } & 28.5 & 1,175 & -- & -- & - & \\
\hline
\end{tabular}



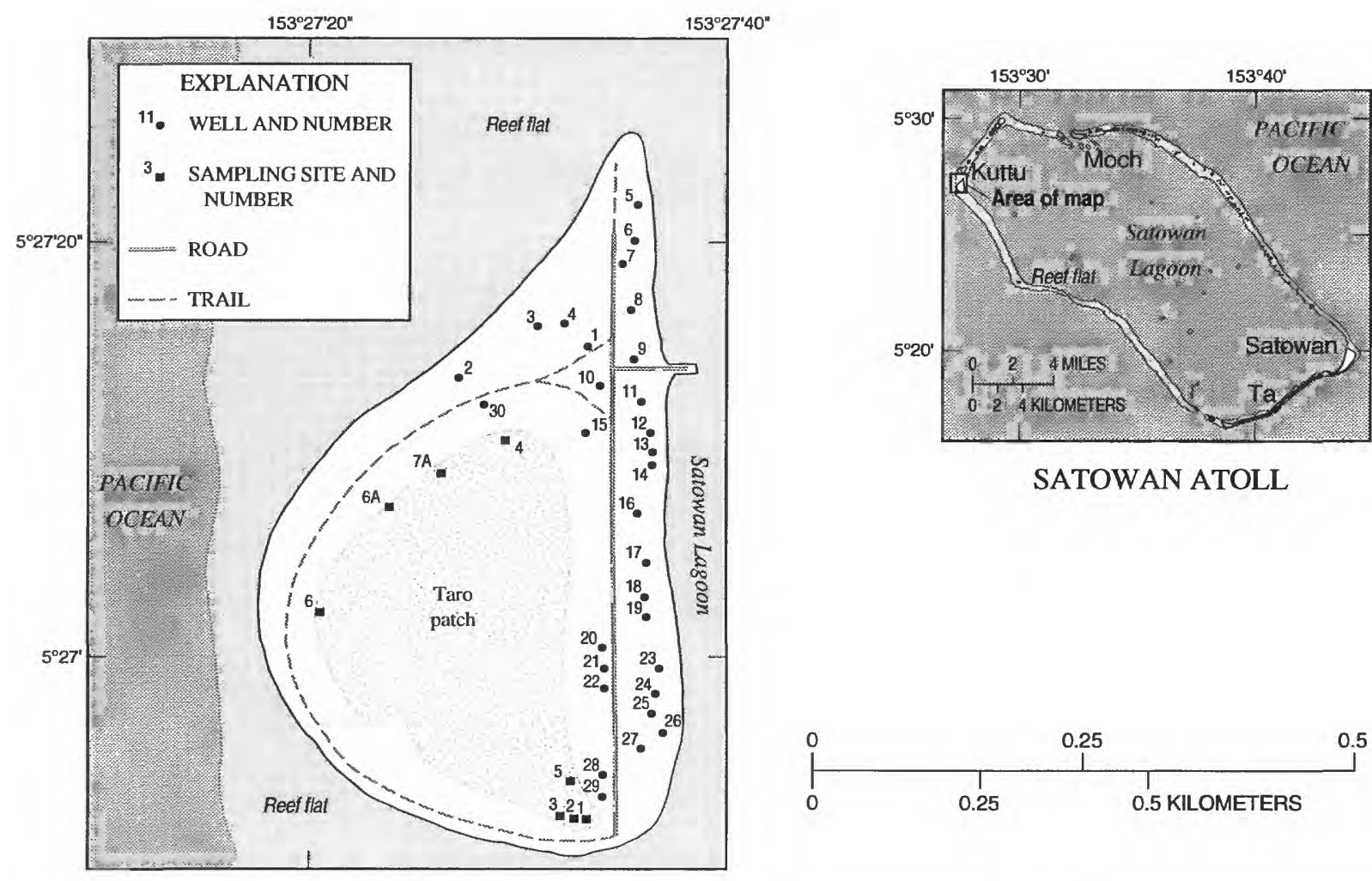

SATOWAN ATOLL
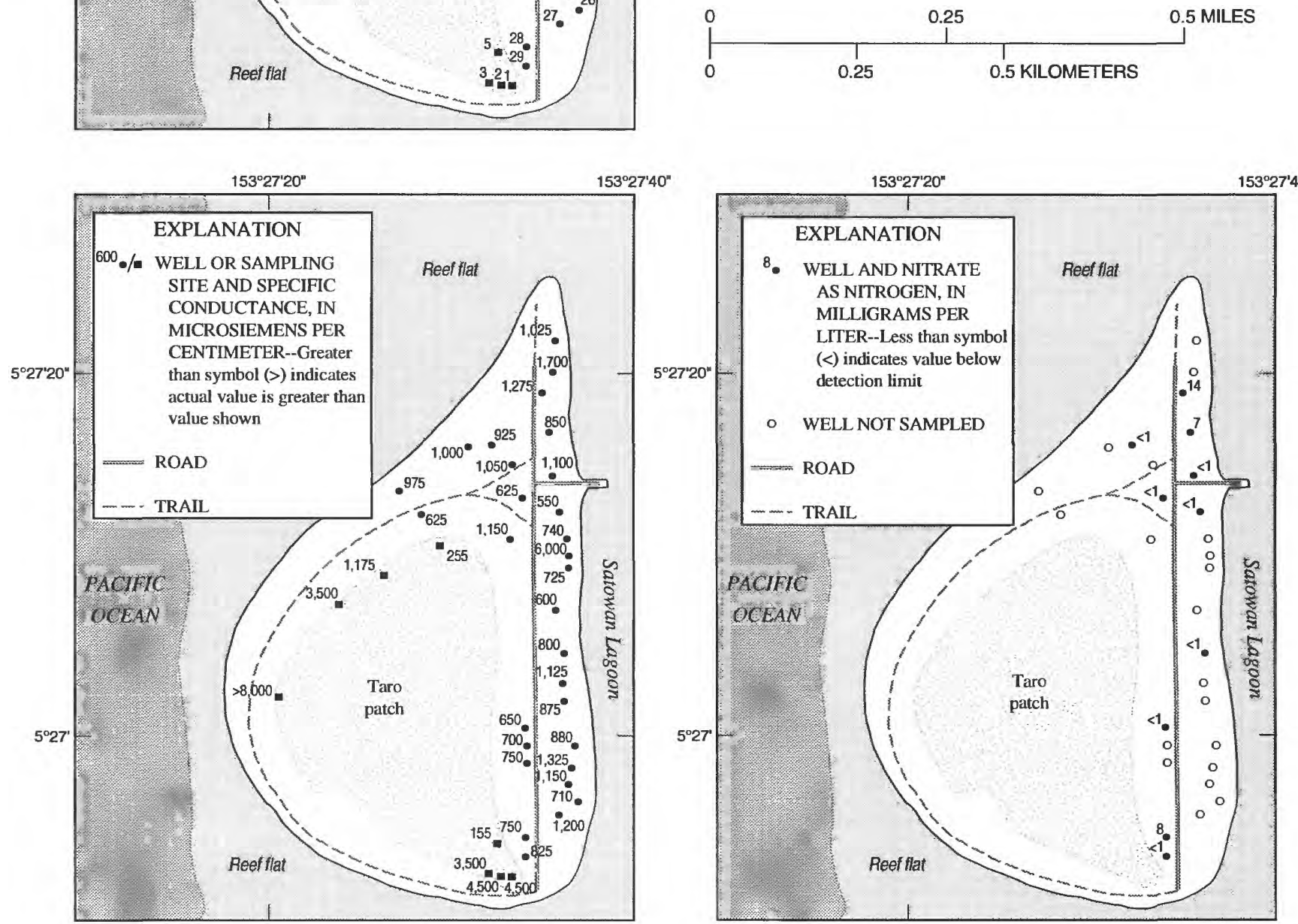

Figure 35. Well locations, specific conductance, and nitrate concentrations for Kuttu Island, Satowan Atoll, Mortlock Island Group, Chuuk State, 1984. 


\section{Moch Island}

The population of Moch Island at Satowan Atoll is about 622 (Truk State Office of Planning and Statistics, 1981). Well locations, sampling sites, specific conductance, and nitrate concentrations for Moch Island are shown in figure 36 . Selected physical and water-quality data for the 30 dug wells and 8 taro patch sites sampled are given in table 28 . The mean specific conductance for water from 30 wells was $1,080 \mu \mathrm{S} / \mathrm{cm}$ and ranged from 390 to $3,000 \mu \mathrm{S} / \mathrm{cm}$. On the basis of the estimated chloride concentrations from specific conductance data, water from 2 of the 30 wells sampled (wells 10 and 11) exceeded the WHO (1984) guideline for chloride. Nine of the 30 wells sampled were field tested for nitrate concentration. Water from 5 of the 30 wells tested had nitrate concentrations of less than $1 \mathrm{mg} / \mathrm{L}$ (as $\mathrm{N}$ ); the remaining samples had concentrations ranging from 3 to $8 \mathrm{mg} / \mathrm{L}$ (as $\mathrm{N}$ ).

Table 28. Selected physical and water-quality data from dug wells and taro patch sites, February 18, 1984, Moch Island, Satowan Atoll, Mortlock Island Group

$\left[{ }^{\circ} \mathrm{C}\right.$, degrees Celsius; $\mu \mathrm{S} / \mathrm{cm}$, microsiemens per centimeter at $25^{\circ} \mathrm{C} ; \mathrm{Cl}$, chloride; $\mathrm{NO}_{3}$, nitrate; $\mathrm{mg} / \mathrm{L}$, milligrams per liter; --, not measured; <, less than; $>$, greater than]

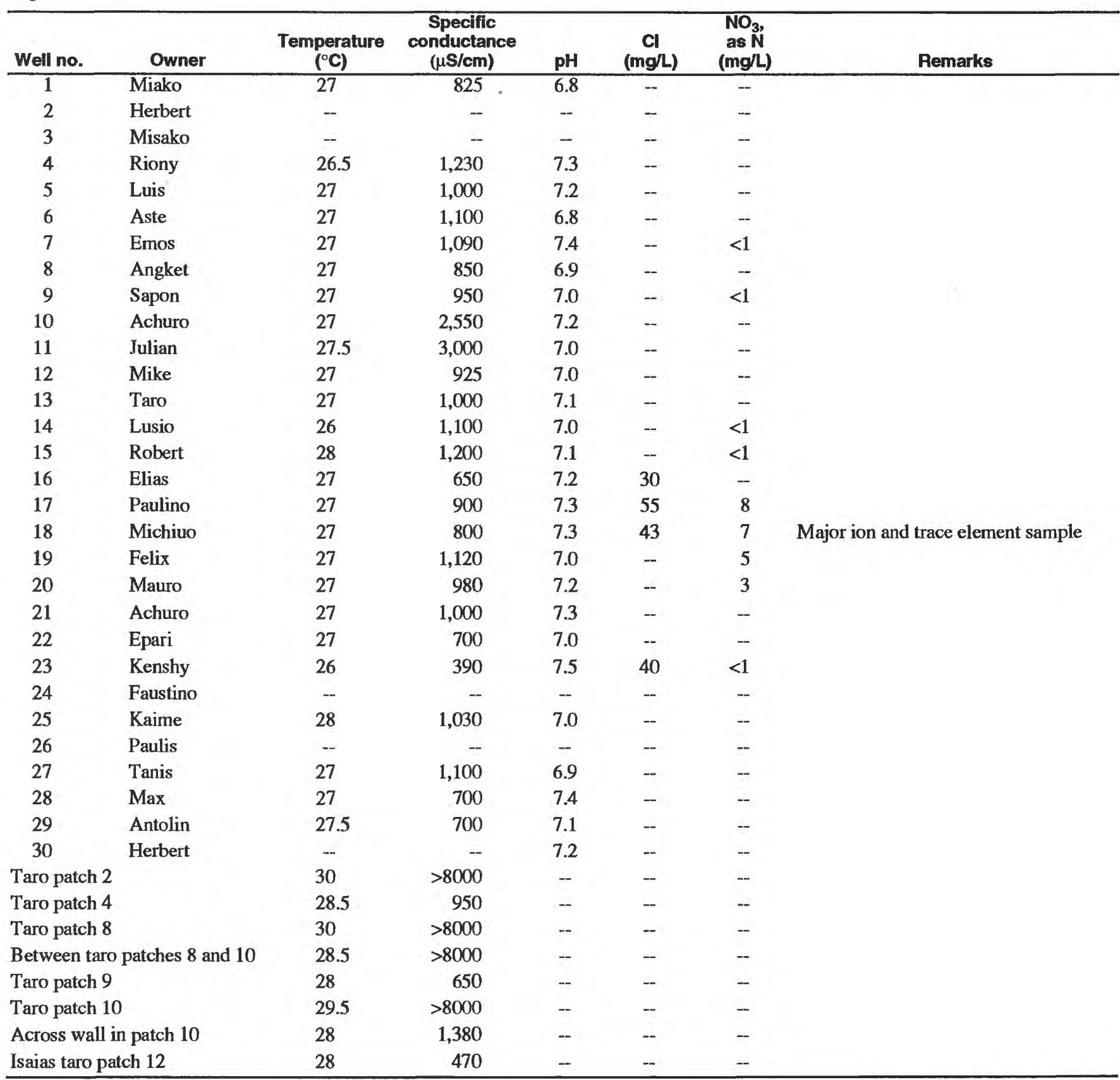



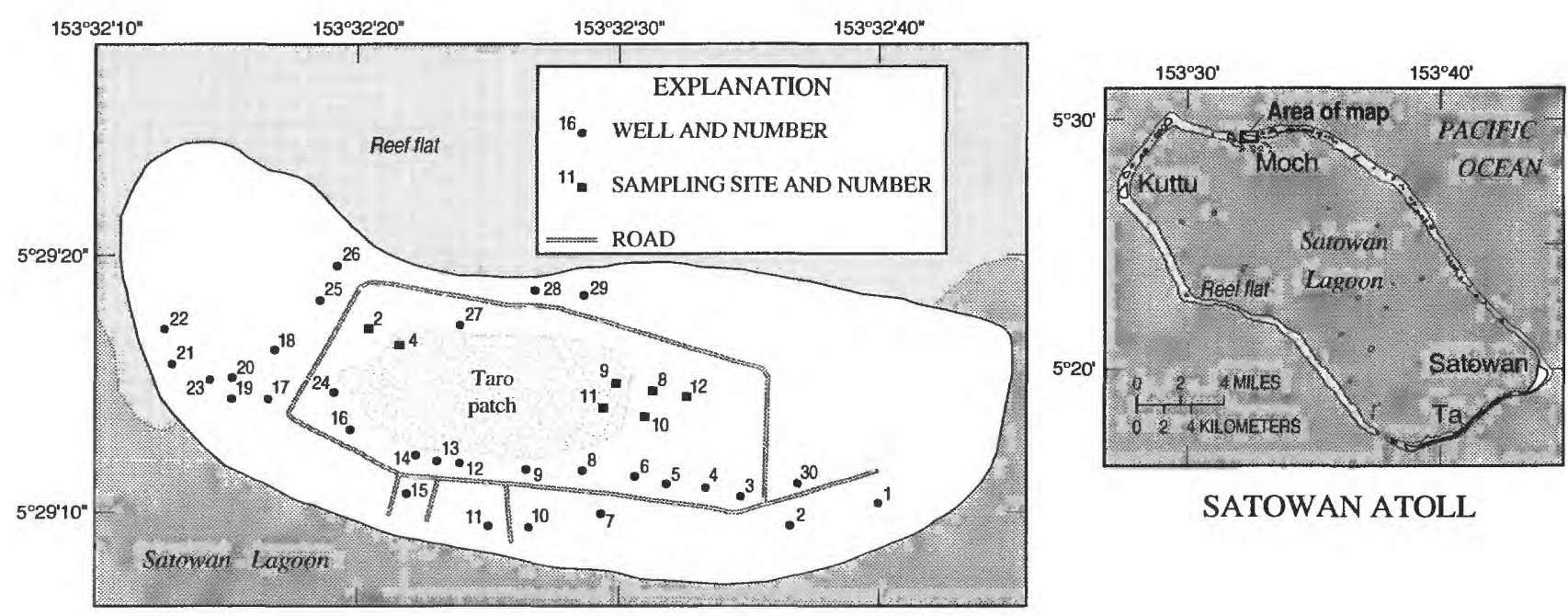

SATOWAN ATOLL
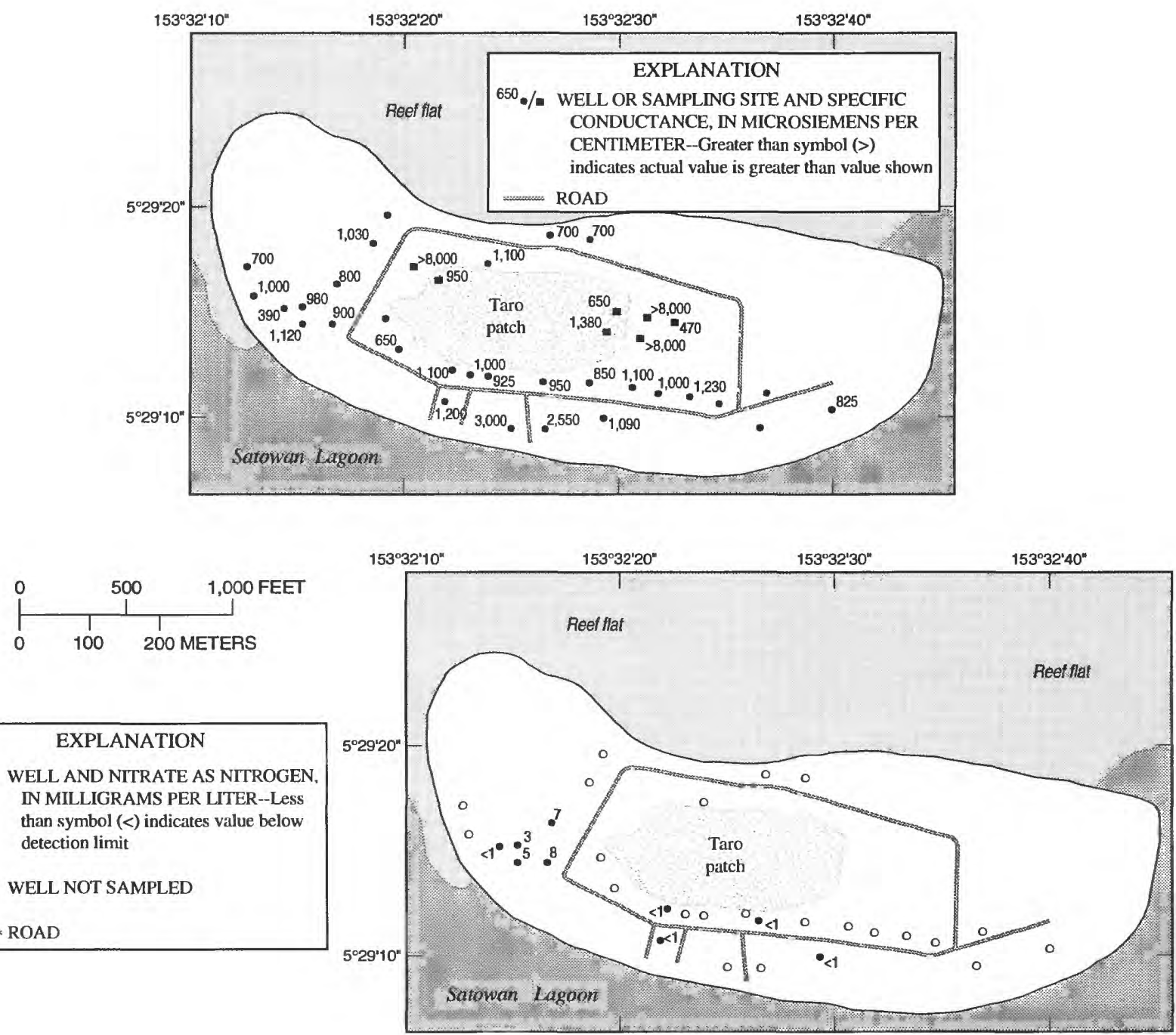

Figure 36. Well locations, specific conductance, and nitrate concentrations for Moch Island, Satowan Atoll, Mortlock Island Group, Chuuk State, 1984. 


\section{SUMMARY}

A ground-water-quality reconnaissance of 24 inhabited outer islands in Chuuk State was made between January 1984 and October 1985. A total of 648 wells were located, described, and sampled for temperature and specific conductance. The nitrate concentration was determined for 308 water samples. To develop a relation between specific conductance and chloride concentration, the chloride concentration was determined for 63 water samples. In addition, 21 water samples were analyzed for major and trace constituent ion concentrations.

Water supplies in the inhabited outer islands of Chuuk State are entirely dependent on rainfall, directly from catchment and indirectly as recharge to ground water. During normal climatic conditions, rainfallcatchment systems are capable of supplying potable water. However, during extended dry periods or droughts, ground water becomes the primary supply for potable water. Under favorable conditions, the freshwater lens acts as a storage reservoir for infiltration from rainfall.

The size and shape of a freshwater lens is controlled by: (1) ground-water recharge and discharge, (2) the hydraulic characteristics (chiefly permeability) of sediments, and (3) island morphology or the size, shape, and presence of a lagoon. Large, circular islands tend to have larger freshwater lenses than small, elongated islands because of their larger storage capacity and less mixing with seawater. The presence of an adjacent lagoon also increases storage in the freshwater lens by acting as a buffer to wave action and allowing accumulation of fine-grained sediments. The thickest part of the lens is associated with fine-grained sediments which, as a result of low permeability, retard mixing of freshwater with seawater.

Chloride and nitrate are the primary constituents that determine the potability of ground water in the inhabited outer islands of Chuuk State. Factors such as shape and size of the island and land-use affect the concentration of these constituents. The source of chloride in ground water is seawater that has been introduced through intrusion, wave washover, and seaspray. Human activities can contaminate ground water with nitrate and pathogenic bacteria. These activities include the disposal of human and animal waste, the application of fertilizers for agriculture, bathing, and the washing of cookware and clothes. Safe-drinking-water guidelines in ground water may be exceeded as a result of these activities.

A statistical summary of specific conductance data collected, including the number of wells sampled, and number of samples exceeding the WHO (1984) drinking-water guideline for chloride is presented in table 29. Although chloride concentration was not determined for all of the wells sampled, it may be estimated from the specific conductance data. For example, values of specific conductance less than $1,500 \mathrm{uS} / \mathrm{cm}$ generally are within the WHO (1984) guideline for chloride (250 $\mathrm{mg} / \mathrm{L}$ ). In the Western Island Group, the guideline for chloride was exceeded for 60 percent of the wells sampled on Tamatam Island, 14 percent of the wells sampled on Pollap Island, and none of the wells sampled on Polowat and Houk Islands. In the Namonweito Island Group, the guideline for chloride was exceeded for 100 percent of the wells sampled on Onou and Unanu Islands, and between 14 and 29 percent of the wells sampled on Onoun, Makur, and Piherarh. In the Hall Island Group, the guideline for chloride was exceeded for 80 percent of the wells sampled on Fananu Island, 40 percent of the wells sampled on Ruo Island, and between 13 and 17 percent of the wells sampled on Nomwin and Murilo Islands. In the Mortlock Island Group, the guideline for chloride was exceeded for between 11 and 33 percent of the wells sampled on Nema, Losap, and Ta Islands, between 2 and 7 percent of the wells sampled on Piis-Emwar, Lukunoch, Satowan, Kuttu, and Moch Islands, and none of the wells sampled on Namoluk and Ettal Islands.

A statistical summary of nitrate-concentration data collected, including the number of wells sampled, and number of samples exceeding the WHO (1984) drinking-water guideline for nitrate is presented in table 30 . The WHO (1984) drinking-water guideline for nitrate is $10 \mathrm{mg} / \mathrm{L}$ (as N). Six of the 308 water samples analyzed for nitrate exceeded the WHO (1984) guideline for nitrate; which represents 5 percent of the wells sampled on Onoun and Piherarh in the Namonweito Island Group, 12 percent of the wells sampled on Nomwin in the Hall Island Group, and 10 percent of the wells sampled on Kuttu in the Mortlock Island Group. 
Table 29. Summary of specific conductance data for islands in the Western, Namonweito, Hall, and Mortlock Island Groups, Chuuk State

[WHO, World Health Organization; $\mu \mathrm{S} / \mathrm{cm}$, microsiemens per centimeter]

\begin{tabular}{|c|c|c|c|c|c|}
\hline \multirow[b]{2}{*}{ Island } & \multirow{2}{*}{$\begin{array}{l}\text { Number of wells } \\
\text { sampled for specific } \\
\text { conductance }\end{array}$} & \multicolumn{3}{|c|}{$\begin{array}{l}\text { Specific conductance } \\
(\mu \mathrm{S} / \mathrm{cm})\end{array}$} & \multirow{2}{*}{$\begin{array}{l}\text { Number of samples } \\
\text { exceedling the } \\
\text { WHO (1984) } \\
\text { guideline for } \\
\text { chloride }\end{array}$} \\
\hline & & Maximum & Minimum & Mean & \\
\hline \multicolumn{6}{|c|}{ Western Island Group } \\
\hline Pollap & 21 & 10,000 & 370 & 1,450 & 3 \\
\hline Tamatam & 5 & 2,400 & 700 & 1,510 & 3 \\
\hline Polowat & 32 & 1,500 & 270 & 790 & 0 \\
\hline Houk & 7 & 1,100 & 390 & 700 & 0 \\
\hline \multicolumn{6}{|c|}{ Namonweito Island Group } \\
\hline Onoun & 41 & 1,750 & 335 & 1,070 & 6 \\
\hline Makur & 6 & 3,000 & 710 & 1,220 & 1 \\
\hline Onou & 2 & 7,500 & 6,000 & 6,750 & 2 \\
\hline Unanu & 8 & 6,000 & 2,000 & 3,340 & 8 \\
\hline Piherarh & 17 & 6,000 & 775 & 1,790 & 5 \\
\hline \multicolumn{6}{|l|}{ Hall Island Group } \\
\hline Nomwin & 24 & 2,700 & 580 & 1,210 & 4 \\
\hline Fananu & 10 & 4,000 & 600 & 2,060 & 8 \\
\hline Murilo & 15 & 1,950 & 650 & 1,120 & 2 \\
\hline Ruo & 10 & 6,000 & 250 & 1,860 & 4 \\
\hline \multicolumn{6}{|c|}{ Mortlock Island Group } \\
\hline Nema & 12 & 8,000 & 750 & 2,650 & 4 \\
\hline Losap & 24 & 3,700 & 620 & 1,260 & 6 \\
\hline Piis-Emwar & 19 & 1,700 & 560 & 940 & 1 \\
\hline Namoluk & 29 & 1,300 & 300 & 660 & 0 \\
\hline Ettal & 38 & 1,330 & 360 & 750 & 0 \\
\hline Lukunoch & 56 & 2,600 & 400 & 830 & 1 \\
\hline Satowan & 64 & 1,700 & 210 & 710 & 1 \\
\hline Oneop & 30 & 1,500 & 330 & 850 & 0 \\
\hline $\mathrm{Ta}$ & 18 & 2,000 & 180 & 850 & 2 \\
\hline Kuttu & 30 & 6,000 & 550 & 1,090 & 2 \\
\hline Moch & 30 & 3,000 & 390 & 1,080 & 2 \\
\hline
\end{tabular}


Table 30. Summary of nitrate-concentration data for islands in the Western, Namonweito, Hall, and Mortlock Island Groups, Chuuk State

[WHO, World Health Organization; mg/L milligrams per liter, <, less than]

\begin{tabular}{|c|c|c|c|c|c|}
\hline \multirow[b]{2}{*}{ Island } & \multirow{2}{*}{$\begin{array}{l}\text { Number of wells } \\
\text { sampled for niltrate } \\
\text { concentration }\end{array}$} & \multicolumn{2}{|c|}{$\begin{array}{c}\text { Nitrate concentration, as N } \\
\text { (mg/L) }\end{array}$} & \multirow{2}{*}{$\begin{array}{l}\text { Number of } \\
\text { samples below } \\
\text { detection } \\
\text { limit }\end{array}$} & \multirow{2}{*}{$\begin{array}{c}\text { Number of samples } \\
\text { exceeding the } \\
\text { WHO (1984) } \\
\text { guldeline for } \\
\text { nitrate }\end{array}$} \\
\hline & & Maximum & Minimum & & \\
\hline \multicolumn{6}{|c|}{ Western Island Group } \\
\hline Pollap & 7 & 5 & $<1$ & 6 & 0 \\
\hline Tamatam & 4 & 7 & $<1$ & 2 & 0 \\
\hline Polowat & 5 & $<1$ & $<1$ & 5 & 0 \\
\hline Houk & 5 & 10 & $<1$ & 2 & 0 \\
\hline \multicolumn{6}{|c|}{ Namonweito Island Group } \\
\hline Onoun & 41 & 15 & $<1$ & 19 & 2 \\
\hline Makur & 6 & 6 & 2 & 0 & 0 \\
\hline Onou & 2 & 2 & 1 & 0 & 0 \\
\hline Unanu & 6 & 5 & $<1$ & 2 & 0 \\
\hline Piherarh & 18 & 11 & 1 & 0 & 1 \\
\hline \multicolumn{6}{|l|}{ Hall Island Group } \\
\hline Nomwin & 17 & 18 & $<1$ & 8 & 2 \\
\hline Fananu & 9 & 4 & $<1$ & 4 & 0 \\
\hline Murilo & 7 & 10 & $<1$ & 3 & 0 \\
\hline Ruo & 4 & 7 & $<1$ & 2 & 0 \\
\hline \multicolumn{6}{|c|}{ Mortlock Island Group } \\
\hline Nema & 6 & 3 & $<1$ & 3 & 0 \\
\hline Losap & 22 & 6 & $<1$ & 15 & 0 \\
\hline Piis-Emwar & 15 & 4 & $<1$ & 12 & 0 \\
\hline Namoluk & 11 & 9 & $<1$ & 8 & 0 \\
\hline Ettal & 6 & 4 & $<1$ & 4 & 0 \\
\hline Lukunoch & 33 & 4 & $<1$ & 32 & 0 \\
\hline Satowan & 38 & 5.5 & $<1$ & 32 & 0 \\
\hline Oneop & 26 & 10 & $<1$ & 21 & 0 \\
\hline $\mathrm{Ta}$ & 1 & $<1$ & $<1$ & 1 & 0 \\
\hline Kuttu & 10 & 14 & $<1$ & 7 & 1 \\
\hline Moch & 9 & 8 & $<1$ & 5 & 0 \\
\hline
\end{tabular}




\section{REFERENCES CITED}

Anthony, S.S., Peterson, F.L., Mackenzie, F.T., and Hamlin, S.N., 1989, Geohydrology of the Laura fresh-water lens, Majuro Atoll: A hydrogeochemical approach: Geological Society of America Bulletin, v. 101, p. 1066-1075.

Ayers, J.F. and Clayshulte, R.N., 1985, Reconnaissance level investigation of salt-water intrusion on Kuttu Island, Satawan Atoll, Truk State: University of Guam, Water and Energy Research Institute Report No. 48, 70 p.

Ayers, J.F. and Vacher, H.L., 1986, Hydrogeology of an atoll island: A conceptual model from detailed study of a Micronesian example: Ground Water, v. 24, p. 185-198.

Hamlin, S.N. and Anthony, S.S., 1987, Ground-water resources of the Laura Area, Majuro Atoll, Marshall Islands: U.S. Geological Survey Water Resources Investigations Report 87-4047, 69 p.

Hem, J.D., 1985, Study and interpretation of the chemical characteristics of natural water: U.S. Geological Survey Water-Supply Paper 2254, 263 p.

National Oceanic and Atmospheric Administration, 1987, Climatological data, Hawaii and Pacific: Department of Commerce, v. 83, nos. 1-12, paginated separately.
National Oceanic and Atmospheric Administration, 1988, Climatological data, Hawaii and Pacific: Department of Commerce, v. 84, nos. 1-12, paginated separately.

Schlanger, S.O., 1963, Subsurface geology of Eniwetok Atoll: U.S. Geological Survey Professional Paper 260-B., p. 991-1066.

Stark, J.T., Paseur, J.E., May, H.G., and Patterson, E.D., 1958, Military geology of Truk Islands, Caroline Islands: Headquarters U.S. Army Pacific, Office of the Engineer, 207 p.

Taylor, R.C., 1973, An atlas of Pacific Islands rainfall: Hawaii Institute of Geophysics, University of Hawaii, HIG-73-9, Data Report No. 25, app. A-E.

Truk State Office of Planning and Statistics, 1981, Statistical Yearbook, Truk State, FSM: Unpublished report, 72 p.

van der Brug, Otto, 1986, The 1983 drought in the Western Pacific: U.S. Geological Survey Open-File Report 85418,89 p.

World Health Organization, 1984, Guidelines for drinkingwater quality, volume 1 of 3: Geneva, Switzerland, $130 \mathrm{p}$. 